Supporting Material belonging to the manuscript entitled:

\title{
Gold Sulfinyl Mesoionic Carbenes: Synthesis, Structure and Catalytic
} Activity

María Frutos, ${ }^{\mathrm{a}, \mathrm{c}}$ Marta G. Avello, ${ }^{\mathrm{a}, \mathrm{c}}$ Alma Viso, ${ }^{\mathrm{a}, \mathrm{c}}$ Roberto Fernández de la Pradilla, María C. de la Torre*, ${ }^{\text {a,c }}$ Miguel A. Sierra, ${ }^{\text {b,c }}$ Heinz Gornitzka, ${ }^{\text {de }}$ and Catherine Hemmert. $^{\mathrm{d}, \mathrm{e}}$

[a] Instituto de Química Orgánica General, Consejo Superior de Investigaciones Científicas (CSIC) Juan de la Cierva 3, 28006-Madrid, Spain

E-mail: mc.delatorre@csic.es

[b] Departamento de Química Orgánica I, Facultad de Química, Universidad Complutense, 28040-Madrid, Spain

E-mail: sierraor@ucm.es

[c] Centro de Innovación en Química Avanzada (ORFEO-CINQA)

[d] CNRS, LCC (Laboratoire de Chimie de Coordination), 205 route de Narbonne, BP 44099, F-31077 Toulouse Cedex 4, France

[e] Université de Toulouse, UPS, INPT, F-31077 Toulouse Cedex 4, France 


\section{General methods}

Unless noted otherwise, all manipulations were carried out under an argon atmosphere using standard Schlenk techniques. DMF and $\mathrm{CH}_{3} \mathrm{CN}$ were dried by passage through solvent purification columns containing activated alumina. $\mathrm{CH}_{2} \mathrm{Cl}_{2}$ was stored on $\mathrm{CaCl}_{2}$ for $24 \mathrm{~h}$ and dried through a distillation with $\mathrm{CaH}_{2}$. Other solvents were HPLC grade and were used without further purification. All reagents were obtained from commercial sources and used without further purification, unless noted otherwise. Flash column chromatography was performed using silica gel (Merck, $\mathrm{n}^{\circ}$ 9385, 230-400 mesh). ${ }^{1} \mathrm{H}$ and ${ }^{13} \mathrm{C}$ NMR spectra were recorded at 300,400 or $500 \mathrm{MHz}\left({ }^{1} \mathrm{H} \mathrm{NMR}\right)$ and at 100 or $125 \mathrm{MHz}\left({ }^{13} \mathrm{C} \mathrm{NMR}\right)$ using $\mathrm{CDCl}_{3}$ and DMSO- $d_{6}$ as solvents with the residual solvent signal as internal reference $\left(\mathrm{CDCl}_{3}, 7.26\right.$ and $\left.77.2 \mathrm{ppm}\right)$ and (DMSO- $d_{6}, 2.50$ and 39.5 ppm). The following abbreviations are used to describe peak patterns when appropriate: s (singlet), d (doublet), t (triplet), q (quadruplet), m (multiplet), and br (broad). Highresolution mass spectrometry (HRMS) by the ESI technique was performed with an Agilent 6500 accurate mass apparatus with a Q-TOF analyser. IR spectra were recorded on a Perkin-Elmer 681 spectrophotometer. Optical rotations were measured on a Jasco P-2000 polarimeter using a sodium lamp. Melting points were determined on a Koffler block.

Alkynes $^{1} \mathbf{1 a}, \mathbf{1 b}, \mathbf{1 c}$ and azides $\mathbf{2} \mathbf{a}^{2}, \mathbf{2 b}-\mathbf{c}^{3}$ were prepared following a procedure previously described:

2d-e were prepared following a modified procedure previously reported: ${ }^{4}$

Amine (16 mmol, 1.00 equiv) was dissolved in THF (20 mL). Ice was then added followed by $\mathrm{HCl} 37 \%$ (3.5 mL). A solution of $\mathrm{NaNO}_{2}$ (24.00 mmol, 1.50 equiv) in $\mathrm{H}_{2} \mathrm{O}$ $(10 \mathrm{~mL})$ was added to the solution. The reaction was stirred at $0^{\circ} \mathrm{C}$ for $15 \mathrm{~min}$. In case of acidic $\mathrm{pH}$ of the solution crude must be neutralized by $\mathrm{NaHCO}_{3} . \mathrm{NaN}_{3}(24 \mathrm{mmol}$, 1.50 equiv) dissolved in $\mathrm{H}_{2} \mathrm{O}$ was added dropwise. The crude reaction was stirred for 1 h. The mixture was extracted with $\mathrm{Et}_{2} \mathrm{O}$ three times. The organic layer is washed with

\footnotetext{
${ }^{1}$ Kosugi, H.; Kitaoka, M.; Tagami, K.; Takahasi, A.; Uda, H. J. Org. Chem. 1987, 52, 1078.

${ }^{2}$ Alvarez, S. G.; Alvarez, M. T. Synthesis 1997, 413.

${ }^{3}$ Suárez, J. R.; Trastoy, B.; Pérez-Ojeda, M. E.; Marín-Barrios, R.; Chiara, J. L. Adv. Synth. Catal. 2010, 352, 2515.

${ }^{4}$ Wilkening, I.; del Signore, G.; Hackenberger, C. P. R. Chem. Commun. 2011, 349.
} 
$\mathrm{HCl} 0.1 \mathrm{M}$ (three times), water (three times), dried over $\mathrm{MgSO}_{4}$ and filtered. All the volatiles were removed under vacuum affording the corresponding azide, which was purified through a short pad of $\mathrm{SiO}_{2}$.

Benzylacetylene $\mathbf{1 0}$ was obtained from commercial source and used without any purification.

\section{General procedure for the synthesis of 1,2,3-triazoles}

A mixture of organic azide (1.20 equiv), alkyne (1.00 equiv), sodium (L)-ascorbate (0.50 equiv) and $\mathrm{CuSO}_{4} \cdot 5 \mathrm{H}_{2} \mathrm{O}$ (0.25 equiv) in DMF was stirred under argon at $\mathrm{rt}$ until completion of the reaction (TLC analysis). The reaction was quenched with water at 0 ${ }^{\circ} \mathrm{C}$ and allowed to reach $\mathrm{rt}$. The mixture was extracted with $\mathrm{CH}_{2} \mathrm{Cl}_{2}$ three times. The organic layer was dried over $\mathrm{MgSO}_{4}$, filtered and the solvent was removed under vacuum to afford the corresponding reaction products, which were purified through a short pad of $\mathrm{SiO}_{2}$.

\section{Preparation of compound 3aa}

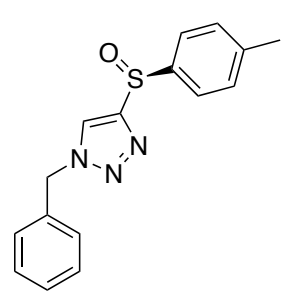

Following the general procedure a mixture of benzyl azide $(525 \mathrm{mg}, 3.95 \mathrm{mmol}, 1.20$ equiv), alkyne 1a (540 mg, $3.29 \mathrm{mmol}, 1.00$ equiv), sodium (L)-ascorbate (326 mg, $1.60 \mathrm{mmol}, 0.50$ equiv) and $\mathrm{CuSO}_{4} \cdot 5 \mathrm{H}_{2} \mathrm{O}(205 \mathrm{mg}, 0.83 \mathrm{mmol}, 0.25$ equiv) in DMF (72 $\mathrm{mL})$ was stirred under $\mathrm{Ar}$ at $\mathrm{rt}$ for $4 \mathrm{~h}$. The resulting residue was purified $\left(\mathrm{SiO}_{2}\right.$, Hex/AcOEt 6:4) to yield 3aa as a white solid (557 mg, 57\%).

${ }^{1} \mathbf{H}$ NMR $\left(400 \mathrm{MHz}, \mathrm{CDCl}_{3}\right): \delta 7.62\left(\mathrm{~s}, 1 \mathrm{H}, \mathrm{N}_{3} \mathrm{C}=\mathrm{CH}\right), 7.52(\mathrm{~d}, J=8.2 \mathrm{~Hz}, 2 \mathrm{H}, \mathrm{Ar} p-$ tolyl), 7.27 (m, 3H, Ar), 7.20 (d, J=8.2 Hz, 2H, Ar p-tolyl), 7.15 (m, 2H, Ar), 5.45 (d, $\left.J=14.7 \mathrm{~Hz}, 1 \mathrm{H}, \mathrm{NCH}_{2}\right), 5.34\left(\mathrm{~d}, J=14.7 \mathrm{~Hz}, 1 \mathrm{H}, \mathrm{NCH}_{2}\right), 2.29$ (s, 3H, $\mathrm{CH}_{3}, p$-tolyl). 
${ }^{13} \mathrm{C}$ NMR $\left(100 \mathrm{MHz}, \mathrm{CDCl}_{3}\right): \delta 153.5\left(\mathrm{C}, \mathrm{N}_{3} \mathrm{C}=\mathrm{CH}\right), 142.2(\mathrm{C}, \mathrm{Ar}), 140.1(\mathrm{C}, \mathrm{Ar})$, 133.5 (C, Ar), 130.2 (2CH, Ar), 129.4 (2CH, Ar), 129.3 (CH, Ar), 128.5 (2CH, Ar), $124.8(2 \mathrm{CH}, \mathrm{Ar}), 123.8\left(\mathrm{CH}, \mathrm{N}_{3} \mathrm{C}=\mathrm{CH}\right), 54.9\left(\mathrm{NCH}_{2}\right), 21.6\left(\mathrm{CH}_{3}, p\right.$-tolyl). IR (KBr): $\boldsymbol{v}_{\text {máx }} 3435,3116,3041,1496,1460,1211,1105,1087,1048,856,814,725,540 .[\alpha]_{D}^{25}+$ 223.0 ( $c$ 0.55, $\mathrm{CHCl}_{3}$ ). MS (ESI) $\mathrm{m} / z$ calculated for $\mathrm{C}_{16} \mathrm{H}_{16} \mathrm{~N}_{3} \mathrm{OS}: 298.1009[\mathrm{M}+\mathrm{H}]^{+}$; found 298.1009 . mp: $132-134{ }^{\circ} \mathrm{C}$.

\section{Preparation of compound 3ab}

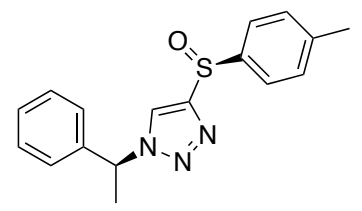

Following the general procedure a mixture of azide $\mathbf{2 b}(645 \mathrm{mg}, 4.38 \mathrm{mmol}, 1.20$ equiv), alkyne 1a (600 mg, $3.65 \mathrm{mmol}, 1.00$ equiv), sodium (L)-ascorbate (362 mg, $1.83 \mathrm{mmol}, 0.50$ equiv) and $\mathrm{CuSO}_{4} \cdot 5 \mathrm{H}_{2} \mathrm{O}(228 \mathrm{mg}, 0.91 \mathrm{mmol}, 0.25$ equiv) in DMF (80 $\mathrm{mL}$ ) was stirred under argon at $\mathrm{rt}$ for $3 \mathrm{~h} 30 \mathrm{~min}$. The resulting residue was purified $\left(\mathrm{SiO}_{2}, \mathrm{Hex} / \mathrm{AcOEt} 7: 3\right)$ to yield $\mathbf{3 a b}$ as a white solid (491 mg, $\left.43 \%\right)$.

${ }^{1} \mathbf{H}$ NMR $\left(400 \mathrm{MHz}, \mathrm{CDCl}_{3}\right): \delta 7.75\left(\mathrm{~s}, 1 \mathrm{H}, \mathrm{N}_{3} \mathrm{C}=\mathrm{CH}\right), 7.62(\mathrm{~d}, J=8.2 \mathrm{~Hz}, 2 \mathrm{H}, \mathrm{Ar} p-$ tolyl), 7.29 (m, 7H, 5H Ar + 2H Ar p-tolyl), 5.80 (q, $J=7.1 \mathrm{~Hz}, 1 \mathrm{H}, \mathrm{CH}), 2.36$ (s, 3H, $\mathrm{CH}_{3}, p$-tolyl), $1.91\left(\mathrm{~d}, J=7.1 \mathrm{~Hz}, 3 \mathrm{H}, \mathrm{CH}_{3}\right) .{ }^{13} \mathbf{C ~ N M R}\left(100 \mathrm{MHz}, \mathrm{CDCl}_{3}\right): \delta 152.7(\mathrm{C}$, $\left.\mathrm{N}_{3} \mathrm{C}=\mathrm{CH}\right), 142.0$ (C, Ar), 139.4 (C, Ar), 138.7 (C, Ar), 130.1 (2CH, Ar), $129.2(2 \mathrm{CH}$, Ar), $128.9(\mathrm{CH}, \mathrm{Ar}), 126.7$ (2CH, Ar), $124.8(2 \mathrm{CH}, \mathrm{Ar}), 122.7\left(\mathrm{CH}, \mathrm{N}_{3} \mathrm{C}=\mathrm{CH}\right), 61.1$ $(\mathrm{CH}), 21.5\left(\mathrm{CH}_{3}, p\right.$-tolyl), $21.3\left(\mathrm{CH}_{3}\right) . \mathbf{I R}(\mathrm{KBr}): \boldsymbol{v}_{\text {máx }}$ 3654, 3103, 1493, 1112, 1084, $1050,819,756,694,523 .[\alpha]_{\mathbf{D}}^{25}+127.7\left(c 0.3, \mathrm{CHCl}_{3}\right) . \mathbf{M S}(\mathrm{ESI}) \mathrm{m} / z$ calculated for $\mathrm{C}_{17} \mathrm{H}_{18} \mathrm{~N}_{3} \mathrm{OS}: 312.1165[\mathrm{M}+\mathrm{H}]^{+}$; found: $312.1176 . \mathbf{m p :} 149-152{ }^{\circ} \mathrm{C}$. 


\section{Preparation of compound 3ac}

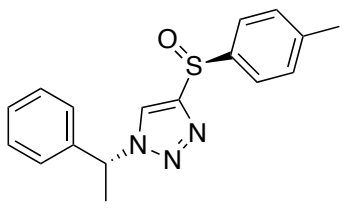

Following the general procedure a mixture of azide $2 \mathrm{c}(645 \mathrm{mg}, 4.38 \mathrm{mmol}, 1.20$ equiv), alkyne 1a (600 mg, $3.65 \mathrm{mmol}, 1.00$ equiv), sodium (L)-ascorbate (362 mg, $1.83 \mathrm{mmol}, 0.50$ equiv) and $\mathrm{CuSO}_{4} \cdot 5 \mathrm{H}_{2} \mathrm{O}(228 \mathrm{mg}, 0.91 \mathrm{mmol}, 0.25$ equiv) in DMF (80 $\mathrm{mL})$ was stirred under argon at $\mathrm{rt}$ for $3 \mathrm{~h}$. The resulting residue was purified $\left(\mathrm{SiO}_{2}\right.$, Hex/AcOEt 7:3) to yield 3ac as a white solid (718 mg, 63\%).

${ }^{1} \mathbf{H}$ NMR $\left(400 \mathrm{MHz}, \mathrm{CDCl}_{3}\right): \delta 7.73\left(\mathrm{~s}, 1 \mathrm{H}, \mathrm{N}_{3} \mathrm{C}=\mathrm{CH}\right), 7.61(\mathrm{~d}, J=8.2 \mathrm{~Hz}, 2 \mathrm{H}, \mathrm{Ar} p-$ tolyl), 7.29 (m, 7H, 5H Ar + 2H Ar p-tolyl), 5.74 (q, $J=7.1 \mathrm{~Hz}, 1 \mathrm{H}, \mathrm{CH}), 2.37$ (s, 3H, $\mathrm{CH}_{3}, p$-tolyl), $1.95\left(\mathrm{~d}, J=7.1 \mathrm{~Hz}, 3 \mathrm{H}, \mathrm{CH}_{3}\right) .{ }^{13} \mathbf{C ~ N M R}\left(100 \mathrm{MHz}, \mathrm{CDCl}_{3}\right): \delta 152.8(\mathrm{C}$, $\left.\mathrm{N}_{3} \mathrm{C}=\mathrm{CH}\right), 142.1$ (C, Ar), $140.0(\mathrm{C}, \mathrm{Ar}), 138.8$ (C, Ar), 130.1 (2CH, Ar), $129.2(2 \mathrm{CH}$, Ar), $129.0(\mathrm{CH}, \mathrm{Ar}), 126.7$ (2CH, Ar), 124.8 (2CH, Ar), $122.9\left(\mathrm{CH}, \mathrm{N}_{3} \mathrm{C}=\mathrm{CH}\right), 61.3$ (CH), $21.5\left(\mathrm{CH}_{3}, p\right.$-tolyl), $21.3\left(\mathrm{CH}_{3}\right) . \mathbf{I R}(\mathbf{K B r}): \boldsymbol{v}_{\text {máx }} 3117,1492,1450,1211,1086$, $1055,809,755,695,552 .[\alpha]_{\mathbf{D}}^{25}+104.5\left(c 1.0, \mathrm{CHCl}_{3}\right) . \mathbf{M S}(\mathrm{ESI}) \mathrm{m} / z$ calculated for $\mathrm{C}_{17} \mathrm{H}_{18} \mathrm{~N}_{3} \mathrm{OS}: 311.1165[\mathrm{M}+\mathrm{H}]^{+}$; found: 312.1165 . mp: $119-121{ }^{\circ} \mathrm{C}$.

\section{Preparation of compound $3 \mathrm{ba}$}

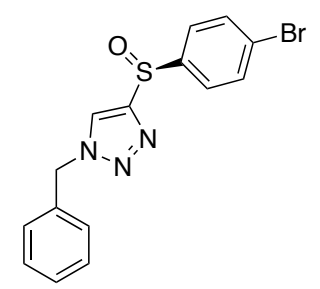

Following the general procedure a mixture of benzyl azide $(229 \mathrm{mg}, 1.72 \mathrm{mmol}, 1.30$ equiv), alkyne $\mathbf{1 b}$ (300 mg, $1.32 \mathrm{mmol}, 1.00$ equiv), sodium (L)-ascorbate (131 mg, $0.66 \mathrm{mmol}, 0.50$ equiv) and $\mathrm{CuSO}_{4} \cdot 5 \mathrm{H}_{2} \mathrm{O}(82 \mathrm{mg}, 0.33 \mathrm{mmol}, 0.25$ equiv) in DMF (11 $\mathrm{mL})$ was stirred under argon at $\mathrm{rt}$ for 3 days. The resulting residue was purified $\left(\mathrm{SiO}_{2}\right.$, Hex/AcOEt from 1:1 to 2:8) to yield 3ba as a white solid (406 mg, 85\%). 
${ }^{1} \mathbf{H}$ NMR $\left(400 \mathrm{MHz}, \mathrm{CDCl}_{3}\right): \delta 7.74\left(\mathrm{~s}, 1 \mathrm{H}, \mathrm{N}_{3} \mathrm{C}=\mathrm{CH}\right), 7.64(\mathrm{~d}, J=8.3 \mathrm{~Hz}, 4 \mathrm{H}, p$ $\left.\mathrm{BrC}_{6} \mathrm{H}_{4}\right), 7.38(\mathrm{~m}, 3 \mathrm{H}, \mathrm{Ar}), 7.27(\mathrm{~m}, 2 \mathrm{H}, \mathrm{Ar}), 5.56\left(\mathrm{~d}, J=14.7 \mathrm{~Hz}, 1 \mathrm{H}, \mathrm{NCH}_{2}\right), 5.46(\mathrm{~d}$, $\left.J=14.7 \mathrm{~Hz}, 1 \mathrm{H}, \mathrm{NCH}_{2}\right) .{ }^{13} \mathrm{C}$ NMR $\left(100 \mathrm{MHz} \mathrm{CDCl}_{3}\right): \delta 152.7\left(\mathrm{C}, \mathrm{N}_{3} \mathrm{C}=\mathrm{CH}\right), 142.4$ (C, Ar), 133.3 (C, Ar), 132.7 (2CH, Ar), 129.5 (2CH, Ar), 129.4 (CH, Ar), 128.6 (2CH, $\mathrm{Ar}), 126.3(2 \mathrm{CH}, \mathrm{Ar}), 126.2(\mathrm{C}, \mathrm{Ar}), 123.9\left(\mathrm{CH}, \mathrm{N}_{3} \mathrm{C}=\mathrm{CH}\right), 55.1\left(\mathrm{NCH}_{2}\right) . \mathbf{I R}(\mathbf{K B r})$ : $\boldsymbol{v}_{\text {máx }}$ 3125, 3065, 2953, 1467, 1384, 12313, 1078, 1046, 1039, 1006, 809, 714, 707.

$[\boldsymbol{\alpha}]_{\mathbf{D}}^{\mathbf{2 5}}+208.0\left(c\right.$ 0.3, $\left.\mathrm{CHCl}_{3}\right)$. MS (ESI) $\mathrm{m} / \mathrm{z}$ calculated for $\mathrm{C}_{15} \mathrm{H}_{13} \mathrm{BrN}_{3} \mathrm{OS}: 363.9937$ $[\mathrm{M}+\mathrm{H}]^{+}$; found $363.9941 . \mathbf{m p :} 129-131^{\circ} \mathrm{C}$.

\section{Preparation of compound 3ca}

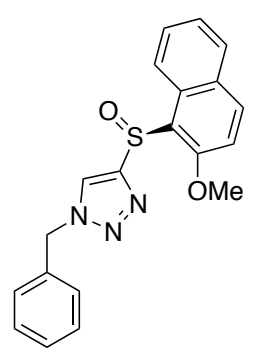

Following the general procedure a mixture of benzyl azide (139 mg, $1.04 \mathrm{mmol}, 1.20$ equiv), alkyne 1c (200 mg, $0.87 \mathrm{mmol}, 1.00$ equiv), sodium (L)-ascorbate (34 mg, 0.17 mmol, 0.20 equiv) and $\mathrm{CuSO}_{4} \cdot 5 \mathrm{H}_{2} \mathrm{O}(21 \mathrm{mg}, 0.09 \mathrm{mmol}, 0.10$ equiv) in DMF (20 mL) was stirred under argon at $\mathrm{rt}$ for $19 \mathrm{~h}$. The resulting residue was purified $\left(\mathrm{SiO}_{2}\right.$, Hex/AcOEt 2:8) to yield 3ca as a white solid (231 mg, $73 \%)$.

${ }^{1} \mathbf{H}$ NMR $\left(400 \mathrm{MHz}, \mathrm{CDCl}_{3}\right): \delta 8.72(\mathrm{dd}, J=8.6 \mathrm{~Hz}, 0.9 \mathrm{~Hz}, 1 \mathrm{H}, \mathrm{Ar}), 7.96(\mathrm{~d}, J=9.0$ $\mathrm{Hz}, 1 \mathrm{H}, \mathrm{Ar}), 7.92\left(\mathrm{~s}, 1 \mathrm{H}, \mathrm{N}_{3} \mathrm{C}=\mathrm{CH}\right), 7.78(\mathrm{dt}, J=8.4 \mathrm{~Hz}, 0.4 \mathrm{~Hz}, 1 \mathrm{H}, \mathrm{Ar}), 7.46$ (ddd, $J=$ $8.5 \mathrm{~Hz}, 6.9 \mathrm{~Hz}, 1.4 \mathrm{~Hz}, 1 \mathrm{H}, \mathrm{Ar}), 7.36$ (m, 4H, Ar), 7.22 (m, 3H, Ar), 5.52 (d, J = 14.8 $\left.\mathrm{Hz}, 1 \mathrm{H}, \mathrm{NCH}_{2}\right), 5.47\left(\mathrm{~d}, J=14.8 \mathrm{~Hz}, 1 \mathrm{H}, \mathrm{NCH}_{2}\right), 3.88\left(\mathrm{~s}, 3 \mathrm{H}, \mathrm{OCH}_{3}\right) .{ }^{13} \mathbf{C} \mathbf{N M R}(100$ $\left.\mathrm{MHz}, \mathrm{CDCl}_{3}\right)$ : $\delta 157.6(\mathrm{C}, \mathrm{Ar}), 151.9\left(\mathrm{C}, \mathrm{N}_{3} \mathrm{C}=\mathrm{CH}\right), 135.4(\mathrm{CH}, \mathrm{Ar}), 134.0(\mathrm{C}, \mathrm{Ar})$, 132.1 (C, Ar), 129.5 (C, Ar), 129.3 (2CH, Ar), 129.1 (CH, Ar), 128.9 (CH, Ar), 128.2 (3CH, Ar), $124.7\left(\mathrm{CH}, \mathrm{N}_{3} \mathrm{C}=\mathrm{CH}\right), 124.6(\mathrm{CH}, \mathrm{Ar}), 122.9(\mathrm{CH}, \mathrm{Ar}), 121.3(\mathrm{C}, \mathrm{Ar}), 113.2$ $(\mathrm{CH}, \mathrm{Ar}), 57.0$ (2-OCH $\mathrm{H}_{3}$ naph), $54.7\left(\mathrm{NCH}_{2}\right)$. IR (KBr): $\boldsymbol{v}_{\text {máx }} 3435,3128,1620,1594$, $1506,1479,1336,1271,1249,1150,1035,815,752,712 .[\alpha]_{\mathbf{D}}^{25}+1.5\left(c 1.2, \mathrm{CHCl}_{3}\right)$. 
MS (ESI) $m / z$ calculated for $\mathrm{C}_{20} \mathrm{H}_{18} \mathrm{~N}_{3} \mathrm{O}_{2} \mathrm{~S}$ : $364.1114[\mathrm{M}+\mathrm{H}]^{+}$; found: 364.1109. mp: $79-82{ }^{\circ} \mathrm{C}$.

\section{Preparation of compound 3cd}

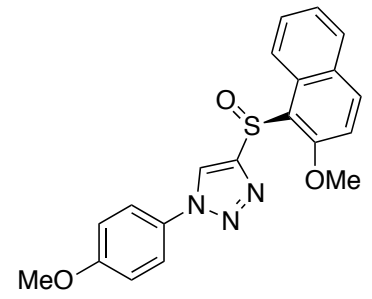

Following the general procedure a mixture of azide $2 \mathbf{d}$ (380 $\mathrm{mg}, 2.54 \mathrm{mmol}, 1.30$ equiv), alkyne 1c (450 mg, $1.95 \mathrm{mmol}, 1.00$ equiv), sodium (L)-ascorbate (194 mg, 0.98 mmol, 0.50 equiv) and $\mathrm{CuSO}_{4} \cdot 5 \mathrm{H}_{2} \mathrm{O}(122 \mathrm{mg}, 0.49 \mathrm{mmol}, 0.25$ equiv) in DMF (44 mL) was stirred under argon at $\mathrm{rt}$ for $2 \mathrm{~h} 30 \mathrm{~min}$. The resulting residue was purified $\left(\mathrm{SiO}_{2}\right.$, Hex/AcOEt 3:7) to yield 3cd as a white solid (687 mg, $93 \%$ ).

${ }^{1} \mathbf{H}$ NMR (400 MHz, $\left.\mathrm{CDCl}_{3}\right): \delta 8.86(\mathrm{dd}, J=8.6 \mathrm{~Hz}, 0.9 \mathrm{~Hz}, 1 \mathrm{H}, \mathrm{Ar}), 8.33(\mathrm{~s}, 1 \mathrm{H}$, $\left.\mathrm{N}_{3} \mathrm{C}=\mathrm{CH}\right), 7.96(\mathrm{~d}, J=9.1 \mathrm{~Hz}, 1 \mathrm{H}, \mathrm{Ar}), 7.77(\mathrm{dt}, J=8.2 \mathrm{~Hz}, 0.7 \mathrm{~Hz}, 1 \mathrm{H}, \mathrm{Ar}), 7.55(\mathrm{~d}, J$ $\left.=9.0 \mathrm{~Hz}, 2 \mathrm{H}, \operatorname{Ar} p-\mathrm{OCH}_{3} \mathrm{C}_{6} \mathrm{H}_{4}\right), 7.50(\mathrm{ddt}, J=8.5 \mathrm{~Hz}, 6.9 \mathrm{~Hz}, 1.4 \mathrm{~Hz}, 1 \mathrm{H}, \mathrm{Ar}), 7.36$ (ddt, $J=8.1 \mathrm{~Hz}, 6.9 \mathrm{~Hz}, 1.1 \mathrm{~Hz}, 1 \mathrm{H}, \mathrm{Ar}), 7.25$ (d, $J=9.1 \mathrm{~Hz}, 1 \mathrm{H}, \mathrm{Ar}), 6.94$ (d, $J=9.0$ $\mathrm{Hz}, 2 \mathrm{H}, \mathrm{Ar} p-\mathrm{OCH}_{3} \mathrm{C}_{6} \mathrm{H}_{4}$ ), 3.97 (s, 3H, 2-OCH 3 naph), 3.80 (s, 3H, p- $\mathrm{OCH}_{3} \mathrm{C}_{6} \mathrm{H}_{4}$ ). ${ }^{13} \mathrm{C}$ NMR $\left(100 \mathrm{MHz}, \mathrm{CDCl}_{3}\right): \delta 160.2(\mathrm{C}, \mathrm{Ar}), 157.4(\mathrm{C}, \mathrm{Ar}), 152.0\left(\mathrm{C}, \mathrm{N}_{3} \mathrm{C}=\mathrm{CH}\right), 135.4$ (CH, Ar), 132.0 (C, Ar), 129.8 (C, Ar), 129.6 (C, Ar), 128.9 (CH, Ar), 128.3 (CH, Ar), $124.6(\mathrm{CH}, \mathrm{Ar}), 123.0(\mathrm{CH}, \mathrm{Ar}), 122.7\left(\mathrm{CH}, \mathrm{N}_{3} \mathrm{C}=\mathrm{CH}\right), 122.3(2 \mathrm{CH}, \mathrm{Ar}), 120.9(\mathrm{C}, \mathrm{Ar})$, $114.9(2 \mathrm{CH}, \mathrm{Ar}), 113.2(\mathrm{CH}, \mathrm{Ar}), 57.1\left(2-\mathrm{OCH}_{3} \mathrm{naph}\right), 55.7\left(p-\mathrm{OCH}_{3} \mathrm{C}_{6} \mathrm{H}_{4}\right) . \mathbf{I R}(\mathbf{K B r})$ : $\boldsymbol{v}_{\text {máx }} 3436,3099,1618,1592,1518,1272,1251,1039,835,823,772 .[\boldsymbol{\alpha}]_{\mathbf{D}}^{\mathbf{2 5}}+13.2(c$ 0.7, $\mathrm{CHCl}_{3}$ ). MS (ESI) $\mathrm{m} / z$ calculated for $\mathrm{C}_{20} \mathrm{H}_{18} \mathrm{~N}_{3} \mathrm{O}_{3} \mathrm{~S}: 380.1063[\mathrm{M}+\mathrm{H}]^{+}$; found 380.1074. For: $\mathrm{C}_{20} \mathrm{H}_{17} \mathrm{~N}_{3} \mathrm{NaO}_{3} \mathrm{~S}: 402.0883\left[\mathrm{M}+\mathrm{Na}^{+}\right]^{+}$; found 402.0892. mp: $123-126{ }^{\circ} \mathrm{C}$. 


\section{Preparation of compound 3ce}

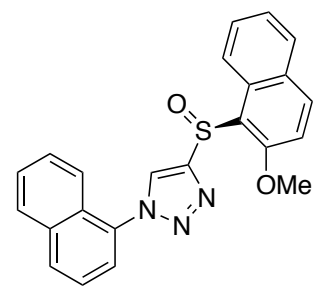

Following the general procedure a mixture of azide $2 \mathbf{e}(400 \mathrm{mg}, 2.36 \mathrm{mmol}, 1.30$ equiv), alkyne 1c (419 mg, $1.82 \mathrm{mmol}, 1.00$ equiv), sodium (L)-ascorbate (180 mg, 0.91 mmol, 0.50 equiv) and $\mathrm{CuSO}_{4} \cdot 5 \mathrm{H}_{2} \mathrm{O}(114 \mathrm{mg}, 0.45 \mathrm{mmol}, 0.25$ equiv) in DMF (40 mL) was stirred under argon at $\mathrm{rt}$ for $2 \mathrm{~h} 30 \mathrm{~min}$. The resulting residue was purified $\left(\mathrm{SiO}_{2}\right.$, Hex/AcOEt 3:7) to yield 3ce as a white solid (541 mg, $57 \%)$.

${ }^{1} \mathrm{H}$ NMR $\left(400 \mathrm{MHz}, \mathrm{CDCl}_{3}\right): \delta 8.94(\mathrm{~d}, J=8.7 \mathrm{~Hz}, 1 \mathrm{H}, \mathrm{Ar}), 8.33\left(\mathrm{~s}, 1 \mathrm{H}, \mathrm{N}_{3} \mathrm{C}=\mathrm{CH}\right)$, 7.99 (m, 2H, Ar), 7.92 (d, $J=8.2 \mathrm{~Hz}, 1 \mathrm{H}, \operatorname{Ar}), 7.81$ (d, $J=8.1 \mathrm{~Hz}, 1 \mathrm{H}, \mathrm{Ar}), 7.55$ (m, $4 \mathrm{H}, \mathrm{Ar}), 7.49$ (m, 2H, Ar), $7.41(\mathrm{ddd}, J=8.1 \mathrm{~Hz}, 6.9 \mathrm{~Hz}, 1.1 \mathrm{~Hz}, 1 \mathrm{H}, \mathrm{Ar}), 7.30$ (d, $J=$ $9.1 \mathrm{~Hz}, 1 \mathrm{H}, \mathrm{Ar}), 4.03$ (s, 3H, 2-OCH $\mathrm{OCH}_{3}$ aph). $\left.{ }^{13} \mathbf{C ~ N M R ~ ( 1 0 0 ~ M H z , ~} \mathrm{CDCl}_{3}\right): \delta 157.6(\mathrm{C}$, Ar), $151.7\left(\mathrm{C}, \mathrm{N}_{3} \mathrm{C}=\mathrm{CH}\right), 135.5$ (CH, Ar), 134.1 (C, Ar), 133.0 (C, Ar), 132.1 (C, Ar), 131.0 (CH, Ar), 129.6 (C, Ar), 129.0 (CH, Ar), 128.4 (CH, Ar), 128.3 (CH, Ar), 128.1 $(\mathrm{CH}, \mathrm{Ar}), 127.3\left(\mathrm{CH}, \mathrm{N}_{3} \mathrm{C}=\mathrm{CH}\right), 127.2(\mathrm{C}, \mathrm{Ar}), 125.0(\mathrm{CH}, \mathrm{Ar}), 124.7(\mathrm{CH}, \mathrm{Ar}), 123.8$ $(\mathrm{CH}, \mathrm{Ar}), 123.0(\mathrm{CH}, \mathrm{Ar}) 121.9(\mathrm{CH}, \mathrm{Ar}) 121.1(\mathrm{C}, \mathrm{Ar}), 121.1(\mathrm{CH}, \mathrm{Ar}), 113.2(\mathrm{CH}$, Ar), 57.2 (2-OCH${ }_{3}$ naph). IR (KBr): $\boldsymbol{v}_{\text {máx }}$ 3436, 3058, 1620, 1594, 1506, 1468, 1430, $1272,1251,1151,1052,1027,802,772 .[\alpha]_{\mathbf{D}}^{25}+229.9\left(c 0.7, \mathrm{CHCl}_{3}\right) . \mathbf{M S}(\mathrm{ESI}) \mathrm{m} / \mathrm{z}$ calculated for $\mathrm{C}_{23} \mathrm{H}_{18} \mathrm{~N}_{3} \mathrm{O}_{2} \mathrm{~S}$ : $400.1114[\mathrm{M}+\mathrm{H}]^{+}$; found: 400.1130. For $\mathrm{C}_{23} \mathrm{H}_{17} \mathrm{~N}_{3} \mathrm{NaO}_{2} \mathrm{~S}$ : $422.0934\left[\mathrm{M}+\mathrm{Na}^{+}\right]^{+}$; found: $422.0946 . \mathbf{m p :} 140-143{ }^{\circ} \mathrm{C}$.

\section{Preparation of compound $11 b$}

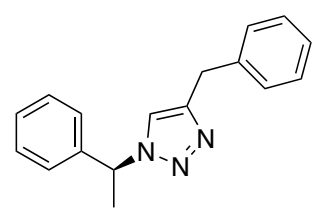

Following the general procedure a mixture of azide $\mathbf{2 b}(646 \mathrm{mg}, 4.39 \mathrm{mmol}, 1.30$ 
equiv), alkyne 10 (390 mg, $3.37 \mathrm{mmol}, 1.00$ equiv), sodium (L)-ascorbate (334 mg, $1.68 \mathrm{mmol}, 0.50$ equiv) and $\mathrm{CuSO}_{4} \cdot 5 \mathrm{H}_{2} \mathrm{O}(210 \mathrm{mg}, 0.84 \mathrm{mmol}, 0.25$ equiv) in DMF (50 $\mathrm{mL})$ was stirred under argon at $\mathrm{rt}$ for $3 \mathrm{~h}$. The resulting residue was purified $\left(\mathrm{SiO}_{2}\right.$, Hex/AcOEt 6:4) to yield $\mathbf{1 1 b}$ as a white solid (777 $\mathrm{mg}, 88 \%$ ).

${ }^{1} \mathbf{H}$ NMR (400 MHz, $\left.\mathrm{CDCl}_{3}\right): \delta 7.27$ (m, 9H, Ar), 7.09 (s, 1H, $\left.\mathrm{N}_{3} \mathrm{C}=\mathrm{CH}\right), 5.73(\mathrm{q}, J=$ $7.1 \mathrm{~Hz}, 1 \mathrm{H}, \mathrm{CH}), 4.06\left(\mathrm{~s}, 2 \mathrm{H}, \mathrm{NCH}_{2}\right), 1.92\left(\mathrm{~d}, J=7.1 \mathrm{~Hz}, 3 \mathrm{H}, \mathrm{CH}_{3}\right) .{ }^{13} \mathbf{C}$ NMR $(100$ $\left.\mathrm{MHz}, \mathrm{CDCl}_{3}\right): \delta 147.6\left(\mathrm{C}, \mathrm{N}_{3} \mathrm{C}=\mathrm{CH}\right), 140.2(\mathrm{C}, \mathrm{Ar}), 139.2(\mathrm{C}, \mathrm{Ar}), 129.0(2 \mathrm{CH}, \mathrm{Ar})$, 128.8 (2CH, Ar), 128.6 (2CH, Ar), 128.5 (CH, Ar), 126.5 (3CH, Ar), $120.2(\mathrm{CH}$, $\left.\mathrm{N}_{3} \mathrm{C}=\mathrm{CH}\right), 60.2(\mathrm{CH}), 32.4\left(\mathrm{NCH}_{2}\right), 21.4\left(\mathrm{CH}_{3}\right)$. IR (KBr): $\boldsymbol{v}_{\text {máx }}$ 3130, 3028, 1495, $1456,1449,1214,1134,1046,727,699,534 .[\alpha]_{\mathbf{D}}^{25}+22.9\left(c 1.0, \mathrm{CHCl}_{3}\right) . \mathbf{M S}(\mathrm{ESI})$ $m / z$ calculated for $\mathrm{C}_{17} \mathrm{H}_{18} \mathrm{~N}_{3}: 264.1495[\mathrm{M}+\mathrm{H}]^{+}$; found: 264.1495 . mp: 80-82 ${ }^{\circ} \mathrm{C}$.

\section{Preparation of compound 11c}

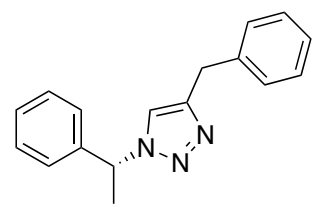

Following the general procedure a mixture of azide 2c $(596 \mathrm{mg}, 4.05 \mathrm{mmol}, 1.20$ equiv), alkyne (392 mg, $3.37 \mathrm{mmol}, 1.00$ equiv), sodium (L)-ascorbate (334 mg, 1.68 mmol, 0.50 equiv) and $\mathrm{CuSO}_{4} \cdot 5 \mathrm{H}_{2} \mathrm{O}(210 \mathrm{mg}, 0.84 \mathrm{mmol}, 0.25$ equiv) in DMF (50 mL) was stirred under argon at $\mathrm{rt}$ for $3 \mathrm{~h}$. The resulting residue was purified $\left(\mathrm{SiO}_{2}\right.$, Hex/AcOEt 7:3) to yield 11c as a white solid (718 mg, 80\%).

${ }^{1} \mathbf{H}$ NMR (400 MHz, $\left.\mathrm{CDCl}_{3}\right): \delta 7.28(\mathrm{~m}, 9 \mathrm{H}, \mathrm{Ar}), 7.10\left(\mathrm{~s}, 1 \mathrm{H}, \mathrm{N}_{3} \mathrm{C}=\mathrm{CH}\right), 5.74(\mathrm{q}, J=$ $7.1 \mathrm{~Hz}, 1 \mathrm{H}, \mathrm{CH}), 4.07\left(\mathrm{~s}, 2 \mathrm{H}, \mathrm{NCH}_{2}\right), 1.93\left(\mathrm{~d}, J=7.1 \mathrm{~Hz}, 3 \mathrm{H}, \mathrm{CH}_{3}\right) .{ }^{13} \mathbf{C}$ NMR (100 $\left.\mathrm{MHz}, \mathrm{CDCl}_{3}\right): \delta 147.6\left(\mathrm{C}, \mathrm{N}_{3} \mathrm{C}=\mathrm{CH}\right), 140.2$ (C, Ar), 139.2 (C, Ar), 129.0 (2CH, Ar), 128.8 (2CH, Ar), 128.6 (2CH, Ar), 128.5 (CH, Ar), 126.5 (3CH, Ar), $120.3(\mathrm{CH}$, $\left.\mathrm{N}_{3} \mathrm{C}=\mathrm{CH}\right), 60.2(\mathrm{CH}), 32.4\left(\mathrm{NCH}_{2}\right), 21.4\left(\mathrm{CH}_{3}\right)$. IR $(\mathbf{K B r}): \boldsymbol{v}_{\text {máx }} 3129,3029,1495$, $1455,1446,1217,1045,727,698 .[\alpha]_{\mathbf{D}}^{25}-21.2\left(c 1.0, \mathrm{CHCl}_{3}\right) . \mathbf{M S}(\mathrm{ESI}) \mathrm{m} / z$ calculated for $\mathrm{C}_{17} \mathrm{H}_{18} \mathrm{~N}_{3}$ : $264.1495[\mathrm{M}+\mathrm{H}]^{+}$; found: 264.1493 . mp: $78-80{ }^{\circ} \mathrm{C}$. 


\section{General procedure for the synthesis of triazolium salts}

Triazole (1.00 equiv) and Meerwein's salt (1.30 equiv) were stirred under argon at $\mathrm{rt}$ in $\mathrm{CH}_{2} \mathrm{Cl}_{2}$ until complete consumption of the starting material ( ${ }^{1} \mathrm{H}$ NMR analysis). The reaction was quenched with some drops of methanol and filtered through a short pad of $\mathrm{NaHCO}_{3}$. The volatiles were removed under vacuum to afford the corresponding reaction product without further purification. In some cases, the product was washed with a mixture of $\mathrm{CH}_{2} \mathrm{Cl}_{2}$ :pentane to remove starting material traces.

\section{Preparation of compound 4aa}

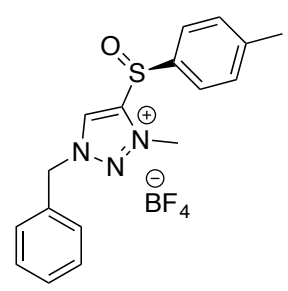

Following the general procedure a mixture of 3aa (72 $\mathrm{mg}, 0.24 \mathrm{mmol}, 1.00$ equiv) and $\mathrm{Me}_{3} \mathrm{OBF}_{4}$ (47 mg, $0.31 \mathrm{mmol}, 1.30$ equiv) in $\mathrm{CH}_{2} \mathrm{Cl}_{2}(16 \mathrm{~mL})$ was stirred under argon at rt overnight. The reaction was quenched with methanol and filtered through a plug of $\mathrm{NaHCO}_{3}$. The volatiles were removed under vacuum to yield 4aa as a white solid (94 mg, $98 \%)$.

${ }^{1} \mathbf{H}$ NMR $\left(400 \mathrm{MHz}, \mathrm{CDCl}_{3}\right): \delta 8.48\left(\mathrm{~s}, 1 \mathrm{H}, \mathrm{N}_{3} \mathrm{C}=\mathrm{CH}\right), 7.72(\mathrm{~d}, J=8.3 \mathrm{~Hz}, 2 \mathrm{H}, \mathrm{Ar} p-$ tolyl), 7.47 (m, 2H, Ar), 7.39 (m, 5H, 3H Ar + 2H Ar p-tolyl), 5.74 (d, J=14.4 Hz, 1H, $\left.\mathrm{NCH}_{2}\right), 5.70\left(\mathrm{~d}, J=14.4 \mathrm{~Hz}, 1 \mathrm{H}, \mathrm{NCH}_{2}\right), 4.28$ (s, 3H, $\left.\mathrm{NCH}_{3}\right), 2.40$ (s, 3H, $\mathrm{CH}_{3}, p$-tolyl). ${ }^{13} \mathrm{C}$ NMR (100 MHz, $\left.\mathrm{CDCl}_{3}\right): \delta 145.8\left(\mathrm{C}, \mathrm{N}_{3} \mathrm{C}=\mathrm{CH}\right), 145.0(\mathrm{C}, \mathrm{Ar}), 134.5(\mathrm{C}, \mathrm{Ar})$, $131.3(2 \mathrm{CH}, \mathrm{Ar}), 131.1\left(\mathrm{CH}, \mathrm{N}_{3} \mathrm{C}=\mathrm{CH}\right), 130.8(\mathrm{C}, \mathrm{Ar}) 130.2(\mathrm{CH}, \mathrm{Ar}), 129.9(2 \mathrm{CH}$, Ar), $129.6(2 \mathrm{CH}, \mathrm{Ar}), 125.6(2 \mathrm{CH}, \mathrm{Ar}), 58.4\left(\mathrm{NCH}_{2}\right), 39.6\left(\mathrm{NCH}_{3}\right), 21.7\left(\mathrm{CH}_{3}, p\right.$-tolyl).

IR (KBr): $\boldsymbol{v}_{\text {máx }} 3420,3147,2923,1060,809,737 .[\boldsymbol{\alpha}]_{\mathbf{D}}^{\mathbf{2 5}}+32.4\left(c 0.2, \mathrm{CHCl}_{3}\right) . \mathbf{M S}$ (ESI) $m / z$ calculated for $\mathrm{C}_{17} \mathrm{H}_{18} \mathrm{~N}_{3} \mathrm{OS}$ : $312.1165\left[\mathrm{M}-\mathrm{BF}_{4}\right]^{+}$; found 312.1175 . mp: 115-118 ${ }^{\circ} \mathrm{C}$. 


\section{Preparation of compound 4ab}

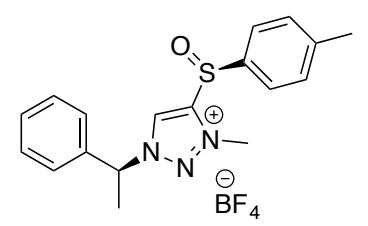

Following the general procedure a mixture of $\mathbf{3 a b}(350 \mathrm{mg}, 1.12 \mathrm{mmol}, 1.00$ equiv) and $\mathrm{Me}_{3} \mathrm{OBF}_{4}$ (216 mg, $1.46 \mathrm{mmol}, 1.30$ equiv) in $\mathrm{CH}_{2} \mathrm{Cl}_{2}(35 \mathrm{~mL})$ was stirred under argon at $\mathrm{rt}$ overnight. The reaction was quenched with methanol and filtered through a plug of $\mathrm{NaHCO}_{3}$. The volatiles were removed under vacuum to yield 4ab as a white solid (421 mg, $91 \%)$.

${ }^{1} \mathbf{H}$ NMR $\left(400 \mathrm{MHz}, \mathrm{CDCl}_{3}\right): \delta 8.48\left(\mathrm{~s}, 1 \mathrm{H}, \mathrm{N}_{3} \mathrm{C}=\mathrm{CH}\right), 7.72(\mathrm{~d}, J=8.0 \mathrm{~Hz}, 2 \mathrm{H}, \mathrm{Ar} p$ tolyl), 7.39 (m, 7H, 5H Ar + 2H Ar p-tolyl), 6.08 (q, J=7.0 Hz, 1H, CH), 4.27 (s, 3H, $\mathrm{NCH}_{3}$ ), $2.38\left(\mathrm{~s}, 3 \mathrm{H}, \mathrm{CH}_{3}, p\right.$-tolyl), $2.02\left(\mathrm{~d}, J=7.0 \mathrm{~Hz}, 3 \mathrm{H}, \mathrm{CH}_{3}\right) .{ }^{13} \mathbf{C} \mathbf{N M R}(100 \mathrm{MHz}$, $\left.\mathrm{CDCl}_{3}\right) \delta 145.4\left(\mathrm{C}, \mathrm{N}_{3} \mathrm{C}=\mathrm{CH}\right), 144.6(\mathrm{C}, \mathrm{Ar}), 136.2(\mathrm{C}, \mathrm{Ar}), 134.9(\mathrm{C}, \mathrm{Ar}), 131.2(2 \mathrm{CH}$, $\mathrm{Ar}), 130.3\left(\mathrm{CH}, \mathrm{N}_{3} \mathrm{C}=\mathrm{CH}\right), 130.0(\mathrm{CH}, \mathrm{Ar}), 129.5$ (2CH, Ar), 127.5 (2CH, Ar), 125.5 (2CH, Ar), $66.2(\mathrm{CH}), 39.7\left(\mathrm{NCH}_{3}\right), 21.6\left(\mathrm{CH}_{3}, p\right.$-tolyl), $20.3\left(\mathrm{CH}_{3}\right)$. IR $(\mathbf{K B r}): v_{\text {máx }}$ $3434,3130,3039,1594,1496,1458,1058,814,732,705,521 .[\alpha]_{\mathbf{D}}^{25}+3.6(c 1.0$, $\mathrm{CHCl}_{3}$ ). MS (ESI) $m / z$ calculated for $\mathrm{C}_{18} \mathrm{H}_{20} \mathrm{~N}_{3} \mathrm{OS}$ : $321.1322\left[\mathrm{M}-\mathrm{BF}_{4}^{-}\right]^{+}$; found: 326.1308. mp: $56-59^{\circ} \mathrm{C}$.

\section{Preparation of compound 4ac}

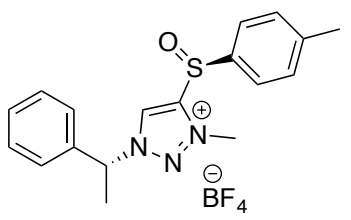

Following the general procedure a mixture of 3ac (274 mg, $0.88 \mathrm{mmol}, 1.00$ equiv) and $\mathrm{Me}_{3} \mathrm{OBF}_{4}$ (169 mg, $1.14 \mathrm{mmol}, 1.30$ equiv) in $\mathrm{CH}_{2} \mathrm{Cl}_{2}(27 \mathrm{~mL})$ was stirred under argon at $\mathrm{rt}$ overnight. The reaction was quenched with methanol and filtered through a plug of $\mathrm{NaHCO}_{3}$. The volatiles were removed under vacuum to yield 4ac as a white solid (307 $\mathrm{mg}, 85 \%)$. 
${ }^{1} \mathbf{H}$ NMR $\left(400 \mathrm{MHz}, \mathrm{CDCl}_{3}\right): \delta 8.43\left(\mathrm{~s}, 1 \mathrm{H}, \mathrm{N}_{3} \mathrm{C}=\mathrm{CH}\right), 7.73(\mathrm{~d}, J=8.0 \mathrm{~Hz}, 2 \mathrm{H}, \mathrm{Ar} p-$ tolyl), 7.38 (m, 7H, 5H Ar + 2H Ar p-tolyl), 6.04 (q, $J=6.8 \mathrm{~Hz}, 1 \mathrm{H}, \mathrm{CH}), 4.30$ (s, 3H, $\mathrm{NCH}_{3}$ ), 2.38 (s, 3H, $\mathrm{CH}_{3}, p$-tolyl), 2.00 (d, $\left.J=6.8 \mathrm{~Hz}, 3 \mathrm{H}, \mathrm{CH}_{3}\right) .{ }^{13} \mathbf{C}$ NMR (100 MHz, $\left.\mathrm{CDCl}_{3}\right)$ : $\delta 145.9\left(\mathrm{C}, \mathrm{N}_{3} \mathrm{C}=\mathrm{CH}\right), 144.7(\mathrm{C}, \mathrm{Ar}), 136.1$ (C, Ar), 134.7 (C, Ar), $131.1(2 \mathrm{CH}$, Ar), $130.2\left(\mathrm{CH}, \mathrm{N}_{3} \mathrm{C}=\mathrm{CH}\right), 129.9(\mathrm{CH}, \mathrm{Ar}), 129.4(2 \mathrm{CH}, \mathrm{Ar}), 127.5$ (2CH, Ar), 125.6 (2CH, Ar), $66.1(\mathrm{CH}), 39.7\left(\mathrm{NCH}_{3}\right), 21.6\left(\mathrm{CH}_{3}, p\right.$-tolyl), $20.4\left(\mathrm{CH}_{3}\right)$. IR $(\mathbf{K B r}): \boldsymbol{v}_{\text {máx }}$ $3427,3127,3039,1594,1496,1457,1249,1087,1058,814,734,704,521 .[\alpha]_{\mathbf{D}}^{25}+$

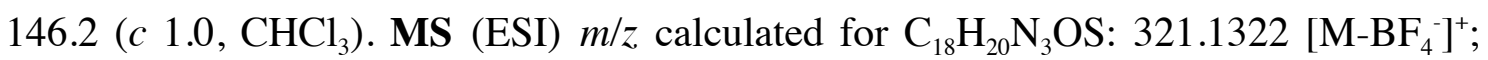
found: 326.1315 . mp: below $30^{\circ} \mathrm{C}$.

\section{Preparation of compound $4 \mathrm{ba}$}

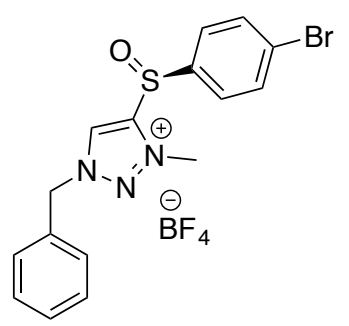

Following the general procedure a mixture of $\mathbf{3 b a}(100 \mathrm{mg}, 0.28 \mathrm{mmol}, 1.00$ equiv) and $\mathrm{Me}_{3} \mathrm{OBF}_{4}$ (61 mg, $0.41 \mathrm{mmol}, 1.50$ equiv) in $\mathrm{CH}_{2} \mathrm{Cl}_{2}(17 \mathrm{~mL})$ was stirred under argon at rt overnight. The reaction was quenched with methanol (5 equiv). The volatiles were removed under vacuum. The crude was precipitated in $\mathrm{Et}_{2} \mathrm{O}$ to yield $\mathbf{4 b a}$ as a white solid (114 mg, 89\%).

${ }^{1} \mathbf{H}$ NMR $\left(400 \mathrm{MHz}, \mathrm{CDCl}_{3}\right): \delta 8.55\left(\mathrm{~s}, 1 \mathrm{H}, \mathrm{N}_{3} \mathrm{C}=\mathrm{CH}\right), 7.75\left(\mathrm{~s}, 4 \mathrm{H}, \mathrm{Ar} p-\mathrm{BrC}_{6} \mathrm{H}_{4}\right), 7.47$ (m, 2H, Ar), 7.40 (m, 3H, Ar), 5.75 (d, $\left.J=14.5 \mathrm{~Hz}, 1 \mathrm{H}, \mathrm{NCH}_{2}\right), 5.71$ (d, $J=14.5 \mathrm{~Hz}$, $\left.1 \mathrm{H}, \mathrm{NCH}_{2}\right), 4.31\left(\mathrm{~s}, 3 \mathrm{H}, \mathrm{NCH}_{3}\right) .{ }^{13} \mathbf{C} \mathbf{N M R}\left(100 \mathrm{MHz}, \mathrm{CDCl}_{3}\right): \delta 145.0\left(\mathrm{C}, \mathrm{N}_{3} \mathrm{C}=\mathrm{CH}\right)$, $137.6(\mathrm{C}, \mathrm{Ar}), 133.9$ (2CH, Ar), $131.5\left(\mathrm{CH}, \mathrm{N}_{3} \mathrm{C}=\mathrm{CH}\right), 130.6(\mathrm{C}, \mathrm{Ar}), 130.3(\mathrm{CH}, \mathrm{Ar})$, 129.9 (2CH, Ar), 129.7 (2CH, Ar), $128.6(\mathrm{C}, \mathrm{Ar}), 127.1(2 \mathrm{CH}, \mathrm{Ar}), 58.6\left(\mathrm{NCH}_{2}\right), 39.8$ $\left(\mathrm{NCH}_{3}\right)$. IR (KBr): $\boldsymbol{v}_{\text {máx }} 3430,3128,1568,1499,1389,1085,1065,822,738 .[\boldsymbol{\alpha}]_{\mathbf{D}}^{\mathbf{2 5}} \ell$

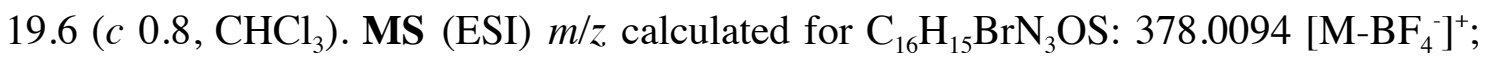
found $378.0097 . \mathbf{m p :} 136-138^{\circ} \mathrm{C}$. 


\section{Preparation of compound $4 \mathrm{ca}$}

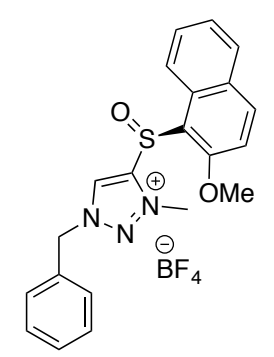

Following the general procedure a mixture of $3 \mathbf{c a}(250 \mathrm{mg}, 0.69 \mathrm{mmol}, 1.00$ equiv) and $\mathrm{Me}_{3} \mathrm{OBF}_{4}$ (132 mg, $0.89 \mathrm{mmol}, 1.30$ equiv) in $\mathrm{CH}_{2} \mathrm{Cl}_{2}(22 \mathrm{~mL})$ was stirred under argon at $\mathrm{rt}$ overnight. The reaction was quenched with methanol and filtered through a plug of $\mathrm{NaHCO}_{3}$. The volatiles were removed under vacuum to yield 4ca as a white solid (321 mg, quantitative).

${ }^{1} \mathrm{H}$ NMR $\left(400 \mathrm{MHz}, \mathrm{CDCl}_{3}\right): \delta 8.58\left(\mathrm{~s}, 1 \mathrm{H}, \mathrm{N}_{3} \mathrm{C}=\mathrm{CH}\right), 8.48(\mathrm{dd}, J=8.8 \mathrm{~Hz}, 0.8 \mathrm{~Hz}$, $1 \mathrm{H}, \mathrm{Ar}), 8.06(\mathrm{~d}, J=9.1 \mathrm{~Hz}, 1 \mathrm{H}, \mathrm{Ar}), 7.78(\mathrm{~d}, J=8.1 \mathrm{~Hz}, 1 \mathrm{H}, \mathrm{Ar}), 7.55(\mathrm{ddd}, J=8.5$ $\mathrm{Hz}, 7.0 \mathrm{~Hz}, 1.4 \mathrm{~Hz}, 1 \mathrm{H}, \mathrm{Ar}), 7.41$ (m, 2H, Ar), 7.36 (ddd, J=8.1 Hz, 6.9 Hz, $1.1 \mathrm{~Hz}$, $1 \mathrm{H}, \mathrm{Ar}), 7.31(\mathrm{~m}, 4 \mathrm{H}, \mathrm{Ar}), 5.65\left(\mathrm{~d}, J=14.4 \mathrm{~Hz}, 1 \mathrm{H}, \mathrm{NCH}_{2}\right), 5.61(\mathrm{~d}, J=14.4 \mathrm{~Hz}, 1 \mathrm{H}$, $\left.\mathrm{NCH}_{2}\right), 4.15$ (s, 3H, $\left.\mathrm{NCH}_{3}\right), 3.93$ (s, 3H, 2-OCH $\mathrm{OCH}_{3}$ na). ${ }^{13} \mathbf{C ~ N M R}\left(100 \mathrm{MHz}, \mathrm{CDCl}_{3}\right): \delta$ $158.7(\mathrm{C}, \mathrm{Ar}), 145.8\left(\mathrm{C}, \mathrm{N}_{3} \mathrm{C}=\mathrm{CH}\right), 138.1$ (CH, Ar), 131.6 (C, Ar), 131.3 (C, Ar), 130.8 $\left(\mathrm{CH}, \mathrm{N}_{3} \mathrm{C}=\mathrm{CH}\right), 129.1(\mathrm{CH}, \mathrm{Ar}), 129.8(\mathrm{CH}, \mathrm{Ar}) 129.7$ (2CH, Ar), $129.5(\mathrm{CH}, \mathrm{Ar})$, 129.4 (2CH, Ar), 128.9 (C, Ar), 125.2 (CH, Ar), 121.2 (CH, Ar), 115.9 (C, Ar), 113.3 $(\mathrm{CH}, \mathrm{Ar}), 58.3\left(\mathrm{NCH}_{2}\right), 57.4\left(2-\mathrm{OCH}_{3} \mathrm{naph}\right), 39.9\left(\mathrm{NCH}_{3}\right)$. IR (KBr): $\boldsymbol{v}_{\text {máx }} 3435,3134$, $1620,1593,1508,1471,1278,1255,1062,820,739 .[\alpha]_{\mathbf{D}}^{25}+94.5\left(c 0.2, \mathrm{CHCl}_{3}\right) . \mathbf{M S}$

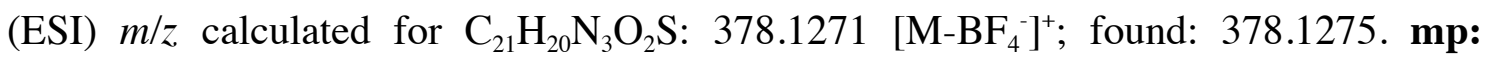
$79-82{ }^{\circ} \mathrm{C}$.

\section{Preparation of compound $4 \mathrm{~cd}$}

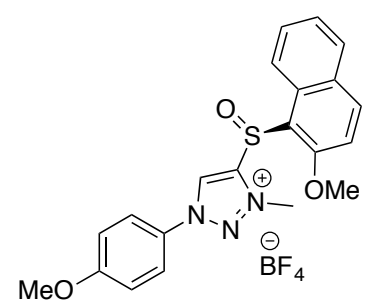


Following the general procedure a mixture of $\mathbf{3 c d}(300 \mathrm{mg}, 0.79 \mathrm{mmol}, 1.00$ equiv) and $\mathrm{Me}_{3} \mathrm{OBF}_{4}$ (152 mg, $1.03 \mathrm{mmol}, 1.30$ equiv) in $\mathrm{CH}_{2} \mathrm{Cl}_{2}(26 \mathrm{~mL})$ was stirred under argon at $\mathrm{rt}$ overnight. The reaction was quenched with methanol and filtered through a plug of $\mathrm{NaHCO}_{3}$. The volatiles were removed under vacuum to yield 4cd as a white solid (366 $\mathrm{mg}, 96 \%)$.

${ }^{1} \mathrm{H}$ NMR $\left(400 \mathrm{MHz}, \mathrm{DMSO}-d_{6}\right): \delta 9.87\left(\mathrm{~s}, 1 \mathrm{H}, \mathrm{N}_{3} \mathrm{C}=\mathrm{CH}\right), 8.67(\mathrm{dd}, J=8.4 \mathrm{~Hz}, 0.8 \mathrm{~Hz}$, $1 \mathrm{H}, \mathrm{Ar}), 8.39$ (d, $J=9.0 \mathrm{~Hz}, 1 \mathrm{H}, \mathrm{Ar}), 8.08(\mathrm{dt}, J=8.0 \mathrm{~Hz}, 1.2 \mathrm{~Hz}, 1 \mathrm{H}, \mathrm{Ar}), 7.89$ (d, $J=$ $\left.9.2 \mathrm{~Hz}, 1 \mathrm{H}, \mathrm{Ar} p-\mathrm{OCH}_{3} \mathrm{C}_{6} \mathrm{H}_{4}\right), 7.69(\mathrm{~m}, 2 \mathrm{H}, \mathrm{Ar}), 7.54$ (ddd, $J=8.1 \mathrm{~Hz}, 6.9 \mathrm{~Hz}, 1.0 \mathrm{~Hz}$, $1 \mathrm{H}, \mathrm{Ar}), 7.22\left(\mathrm{~d}, J=9.2 \mathrm{~Hz}, 2 \mathrm{H}, \mathrm{Ar} p-\mathrm{OCH}_{3} \mathrm{C}_{6} \mathrm{H}_{4}\right), 4.36\left(\mathrm{~s}, 3 \mathrm{H}, \mathrm{NCH}_{3}\right), 4.10(\mathrm{~s}, 3 \mathrm{H}, 2-$ $\mathrm{OCH}_{3}$ naph), 3.86 (s, 3H, $p$ - $\left.\mathrm{OCH}_{3} \mathrm{C}_{6} \mathrm{H}_{4}\right) .{ }^{13} \mathrm{C}$ NMR (125 MHz, DMSO- $\left.d_{6}\right): \delta 161.6(\mathrm{C}$, $\mathrm{Ar}), 158.5(\mathrm{C}, \mathrm{Ar}), 145.6\left(\mathrm{C}, \mathrm{N}_{3} \mathrm{C}=\mathrm{C}\right), 137.7(\mathrm{CH}, \mathrm{Ar}), 130.7(\mathrm{C}, \mathrm{Ar}), 129.5(\mathrm{CH}, \mathrm{Ar})$, $129.2(\mathrm{C}, \mathrm{Ar}), 129.0(\mathrm{CH}, \mathrm{Ar}), 128.7\left(\mathrm{CH}, \mathrm{N}_{3} \mathrm{C}=\mathrm{C}\right), 127.6(\mathrm{C}, \mathrm{Ar}), 124.9(\mathrm{CH}, \mathrm{Ar})$ 123.3 (2CH, Ar), 121.9 (CH, Ar), 116.3 (C, Ar), 115.3 (2CH, Ar), 114.1 (CH, Ar), 57.3 $\left(\mathrm{CH}_{3}, 2-\mathrm{OCH}_{3}\right.$ naph $), 56.0\left(\mathrm{CH}_{3}, p-\mathrm{OCH}_{3} \mathrm{C}_{6} \mathrm{H}_{4}\right), 39.5\left(\mathrm{NCH}_{3}\right.$ overlapped with DMSO- $\left.d_{6}\right)$. IR (KBr): $\boldsymbol{v}_{\text {máx }} 3436,3120,1594,1509,1470,1276,1256,1183,1059,835,820,755$, 608. $[\alpha]_{\mathbf{D}}^{25}+273.3\left(c 0.5, \mathrm{CHCl}_{3}\right) . \mathbf{M S}(\mathrm{ESI}) \mathrm{m} / \mathrm{z}$, calculated for $\mathrm{C}_{21} \mathrm{H}_{20} \mathrm{~N}_{3} \mathrm{O}_{3} \mathrm{~S}: 394.1220$ [M-BF $\left.{ }_{4}^{-}\right]^{+}$; found: $394.1220 . \mathbf{m p :} 97-100{ }^{\circ} \mathrm{C}$.

\section{Preparation of compound 4ce}

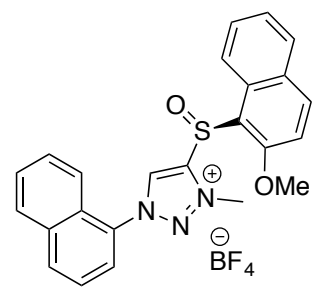

Following the general procedure a mixture of 3ce (400 mg, $1.00 \mathrm{mmol}, 1.00$ equiv) and $\mathrm{Me}_{3} \mathrm{OBF}_{4}$ (193 mg, $1.30 \mathrm{mmol}, 1.30$ equiv) in $\mathrm{CH}_{2} \mathrm{Cl}_{2}(30 \mathrm{~mL})$ was stirred under argon at $\mathrm{rt}$ overnight. The reaction was quenched with methanol and filtered through a plug of $\mathrm{NaHCO}_{3}$. The volatiles were removed under vacuum to yield 4ce as a white solid (501 mg, quantitative). 
${ }^{1} \mathbf{H}$ NMR (400 MHz, $\left.\mathrm{CDCl}_{3}\right): \delta 8.48(\mathrm{~d}, J=8.6 \mathrm{~Hz}, 1 \mathrm{H}, \mathrm{Ar}), 8.33\left(\mathrm{~s}, 1 \mathrm{H}, \mathrm{N}_{3} \mathrm{C}=\mathrm{CH}\right)$, $8.07(\mathrm{~d}, J=9.2 \mathrm{~Hz}, 1 \mathrm{H}, \mathrm{Ar}), 7.94(\mathrm{~d}, J=8.2 \mathrm{~Hz}, 1 \mathrm{H}, \mathrm{Ar}), 7.83$ (m, $2 \mathrm{H}, \mathrm{Ar}), 7.74$ (d, $J=$ $8.1 \mathrm{~Hz}, 1 \mathrm{H}, \mathrm{Ar}), 7.53$ (m, 3H, Ar), 7.43 (m, 2H, Ar), 7.32 (m, 2H, Ar), 4.38 (s, 3H, $\left.\mathrm{NCH}_{3}\right), 4.01$ (s, 3H, 2-OCH 3 naph). ${ }^{13} \mathrm{C}$ NMR (100 MHz, $\left.\mathrm{CDCl}_{3}\right): \delta 159.2(\mathrm{C}, \mathrm{Ar})$, $146.9\left(\mathrm{C}, \mathrm{N}_{3} \mathrm{C}=\mathrm{CH}\right), 138.2(\mathrm{CH}, \mathrm{Ar}), 133.8(\mathrm{C}, \mathrm{Ar}), 133.0(\mathrm{CH}, \mathrm{Ar}), 132.3(\mathrm{CH}$, $\left.\mathrm{N}_{3} \mathrm{C}=\mathrm{CH}\right), 131.6(\mathrm{C}, \mathrm{Ar}), 130.6(\mathrm{C}, \mathrm{Ar}), 129.7(\mathrm{CH}, \mathrm{Ar}), 129.6(\mathrm{CH}, \mathrm{Ar}), 129.3(\mathrm{CH}$, Ar), 129.1 (C, Ar), 128.7 (CH, Ar), 127.8 (CH, Ar), 126.7 (C, Ar), 125.5 (CH, Ar), 125.2 (CH, Ar), 125.1 (CH, Ar), 121.5 (CH, Ar), 120.5 (CH, Ar), 116.1 (C, Ar), 113.6 $(\mathrm{CH}, \mathrm{Ar}), 57.2\left(2-\mathrm{OCH}_{3} \mathrm{naph}\right), 40.1\left(\mathrm{NCH}_{3}\right)$. IR (KBr): $\boldsymbol{v}_{\text {máx }} 3435,3066,2950,1593$, $1508,1470,1277,1254,1059,806,772,651,461 .[\alpha]_{\mathbf{D}}^{25}+174.3\left(c 0.8, \mathrm{CHCl}_{3}\right) . \mathbf{M S}$ (ESI) $m / z$ calculated for $\mathrm{C}_{24} \mathrm{H}_{20} \mathrm{~N}_{3} \mathrm{O}_{2} \mathrm{~S}: 414.1271\left[\mathrm{M}-B F_{4}^{-}\right]^{+}$; found: 414.1281. mp: 109$112^{\circ} \mathrm{C}$.

\section{Preparation of compound $12 \mathrm{~b}$}

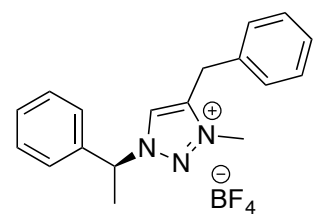

Following the general procedure a mixture of $\mathbf{1 1 b}(643 \mathrm{mg}, 2.44 \mathrm{mmol}, 1.00$ equiv) and $\mathrm{Me}_{3} \mathrm{OBF}_{4}$ (470 mg, $3.17 \mathrm{mmol}, 1.30$ equiv) in $\mathrm{CH}_{2} \mathrm{Cl}_{2}(75 \mathrm{~mL})$ was stirred under argon at $\mathrm{rt}$ overnight. The reaction was quenched with methanol and filtered through a plug of $\mathrm{NaHCO}_{3}$. The volatiles were removed under vacuum to yield 12b as a white solid (740 $\mathrm{mg}, 83 \%)$.

${ }^{1} \mathbf{H}$ NMR $\left(400 \mathrm{MHz}, \mathrm{CDCl}_{3}\right): \delta 8.10\left(\mathrm{~s}, 1 \mathrm{H}, \mathrm{N}_{3} \mathrm{C}=\mathrm{CH}\right), 7.39(\mathrm{~m}, 5 \mathrm{H}, \mathrm{Ar}), 7.28(\mathrm{~m}, 5 \mathrm{H}$, Ar), $5.91(\mathrm{q}, J=7.0 \mathrm{~Hz}, 1 \mathrm{H}, \mathrm{CH}), 4.19\left(\mathrm{~s}, 2 \mathrm{H}, \mathrm{NCH}_{2}\right), 4.11\left(\mathrm{~s}, 3 \mathrm{H}, \mathrm{NCH}_{3}\right), 2.01(\mathrm{~d}, J=$ $\left.7.0 \mathrm{~Hz}, 3 \mathrm{H}, \mathrm{CH}_{3}\right) .{ }^{13} \mathrm{C}$ NMR $\left(100 \mathrm{MHz}, \mathrm{CDCl}_{3}\right): \delta 144.2\left(\mathrm{C}, \mathrm{N}_{3} \mathrm{C}=\mathrm{CH}\right), 136.7(\mathrm{C}, \mathrm{Ar})$, 133.2 (C, Ar), 129.9 (CH, Ar), 129.6 (2CH, Ar), 129.5 (2CH, Ar), 129.1 (2CH, Ar), $128.1(\mathrm{CH}, \mathrm{Ar}), 127.9\left(\mathrm{CH}, \mathrm{N}_{3} \mathrm{C}=\mathrm{CH}\right), 127.3(2 \mathrm{CH}, \mathrm{Ar}), 65.1(\mathrm{CH}), 38.1\left(\mathrm{NCH}_{3}\right), 29.3$ $\left(\mathrm{NCH}_{2}\right), 20.6\left(\mathrm{CH}_{3}\right) . \mathbf{I R}(\mathbf{K B r}): \boldsymbol{v}_{\text {máx }} 3430,3052,1455,1083,749,702 .[\boldsymbol{\alpha}]_{\mathbf{D}}^{25}+7.9(c$ 1.0, $\mathrm{CHCl}_{3}$ ). MS (ESI) $\mathrm{m} / \mathrm{z}$ calculated for $\mathrm{C}_{18} \mathrm{H}_{20} \mathrm{~N}_{3}: 278.1652\left[\mathrm{M}-\mathrm{BF}_{4}\right]^{+}$; found: 278.1656. mp: $80-82{ }^{\circ} \mathrm{C}$. 


\section{Preparation of compound 12c}

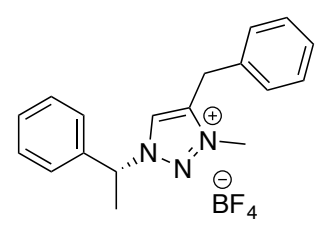

Following the general procedure a mixture of $11 \mathrm{c}(560 \mathrm{mg}, 2.13 \mathrm{mmol}, 1.00$ equiv) and $\mathrm{Me}_{3} \mathrm{OBF}_{4}$ (409 mg, $2.76 \mathrm{mmol}, 1.30$ equiv) in $\mathrm{CH}_{2} \mathrm{Cl}_{2}(65 \mathrm{~mL})$ was stirred under argon at $\mathrm{rt}$ overnight. The reaction was quenched with methanol and filtered through a plug of $\mathrm{NaHCO}_{3}$. The volatiles were removed under vacuum to yield $\mathbf{1 2 c}$ as a white solid $(720$ mg, 90\%).

${ }^{1}$ H NMR $\left(400 \mathrm{MHz}, \mathrm{CDCl}_{3}\right): \delta 8.10\left(\mathrm{~s}, 1 \mathrm{H}, \mathrm{N}_{3} \mathrm{C}=\mathrm{CH}\right), 7.36(\mathrm{~m}, 5 \mathrm{H}, \mathrm{Ar}), 7.26(\mathrm{~m}, 5 \mathrm{H}$, $\operatorname{Ar}), 5.91(\mathrm{q}, J=7.0 \mathrm{~Hz}, 1 \mathrm{H}, \mathrm{CH}), 4.18\left(\mathrm{~s}, 2 \mathrm{H}, \mathrm{NCH}_{2}\right), 4.10\left(\mathrm{~s}, 3 \mathrm{H}, \mathrm{NCH}_{3}\right), 1.99(\mathrm{~d}, J=$ $\left.7.0 \mathrm{~Hz}, 3 \mathrm{H}, \mathrm{CH}_{3}\right) .{ }^{13} \mathrm{C}$ NMR $\left(100 \mathrm{MHz}, \mathrm{CDCl}_{3}\right): \delta 144.1\left(\mathrm{C}, \mathrm{N}_{3} \mathrm{C}=\mathrm{CH}\right), 136.7(\mathrm{C}, \mathrm{Ar})$, 133.2 (C, Ar), 129.8 (CH, Ar), 129.5 (2CH, Ar), 129.4 (2CH, Ar), 129.1 (2CH, Ar), $128.0(\mathrm{CH}, \mathrm{Ar}), 127.8\left(\mathrm{CH}, \mathrm{N}_{3} \mathrm{C}=\mathrm{CH}\right), 127.2(2 \mathrm{CH}, \mathrm{Ar}), 64.9(\mathrm{CH}), 38.1\left(\mathrm{NCH}_{3}\right), 29.2$ $\left(\mathrm{NCH}_{2}\right), 20.5\left(\mathrm{CH}_{3}\right)$. IR (KBr): $\boldsymbol{v}_{\text {máx }}$ 3430, 3128, 3034, 1592, 1497, 1455, 1278, 1071, 749, 701, 522. $[\boldsymbol{\alpha}]_{\mathbf{D}}^{25}-8.0\left(c 1.0, \mathrm{CHCl}_{3}\right)$. MS (ESI) $\mathrm{m} / z$ calculated for $\mathrm{C}_{18} \mathrm{H}_{20} \mathrm{~N}_{3}$ :

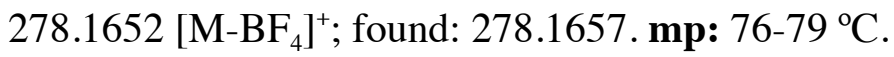

\section{General procedure for the synthesis of gold carbenes}

In a schlenk flask charged with $4 \AA$ molecular sieves, a mixture of triazolium salt (1.00 equiv), $\mathrm{NMe}_{4} \mathrm{Cl}$ (1.50 equiv) and $\mathrm{Ag}_{2} \mathrm{O}$ (0.75 equiv) was stirred at $\mathrm{rt}$ in the dark in $\mathrm{CH}_{3} \mathrm{CN}: \mathrm{CH}_{2} \mathrm{Cl}_{2}$ until the formation of the silver carbene $\left({ }^{1} \mathrm{H}\right.$ NMR analysis). The $\left[\mathrm{AuCl}\left(\mathrm{SMe}_{2}\right)\right]$ (1.00 equiv) was added and the reaction was stirred at $\mathrm{rt}$ until completion of the reaction ( ${ }^{1} \mathrm{H}$ NMR analysis). The reaction was filtered through a pad of Celite and the volatiles were removed under vacuum to afford the corresponding reaction products, which were purified through a short pad of $\mathrm{SiO}_{2}$. 


\section{Preparation of compound 5aa}

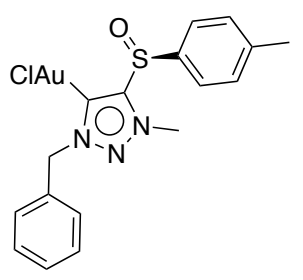

Following the general procedure a mixture of $4 \mathbf{a a}(255 \mathrm{mg}, 0.64 \mathrm{mmol}, 1.00$ equiv), $\mathrm{NMe}_{4} \mathrm{Cl}$ (105 mg, $0.96 \mathrm{mmol}, 1.50$ equiv) and $\mathrm{Ag}_{2} \mathrm{O}$ (111 mg, $0.48 \mathrm{mmol}, 0.75$ equiv) in $\mathrm{CH}_{3} \mathrm{CN}: \mathrm{CH}_{2} \mathrm{Cl}_{2}$ 1:1 (13 mL) was stirred under argon at $\mathrm{rt}$ for $3 \mathrm{~h}$. [AuCl$\left.\left(\mathrm{SMe}_{2}\right)\right](188$ $\mathrm{mg}, 0.64 \mathrm{mmol}, 1.00$ equiv) was added and the reaction was stirred for two more hours. The resulting residue was purified $\left(\mathrm{SiO}_{2}, \mathrm{Hex} / \mathrm{AcOEt}\right.$ 3:7) to yield 5aa as a white solid (243 mg, 98\%).

${ }^{1} \mathbf{H}$ NMR $\left(400 \mathrm{MHz}, \mathrm{CDCl}_{3}\right): \delta 7.67(\mathrm{~d}, J=7.9 \mathrm{~Hz}, 2 \mathrm{H}, \mathrm{Ar} p$-tolyl), 7.52 (m, 2H, Ar), $7.37(\mathrm{~m}, 5 \mathrm{H}, 3 \mathrm{H} \mathrm{Ar}+2 \mathrm{H} p$-tolyl $), 5.67\left(\mathrm{~d}, J=14 \mathrm{~Hz}, 1 \mathrm{H}, \mathrm{NCH}_{2}\right), 5.61(\mathrm{~d}, J=14 \mathrm{~Hz}$, $\left.1 \mathrm{H}, \mathrm{NCH}_{2}\right), 4.15\left(\mathrm{~s}, 3 \mathrm{H}, \mathrm{NCH}_{3}\right), 2.41$ (s, 3H, $\mathrm{CH}_{3}, p$-tolyl). ${ }^{13} \mathbf{C}$ NMR (100 MHz, $\left.\mathrm{CDCl}_{3}\right): \delta 160.7\left(\mathrm{C}, \mathrm{N}_{3} \mathrm{C}=C \mathrm{Au}\right), 146.8\left(\mathrm{C}, \mathrm{N}_{3} \mathrm{C}=\mathrm{CAu}\right), 143.1(\mathrm{C}, \mathrm{Ar}), 136.9(\mathrm{C}, \mathrm{Ar} \mathrm{S})$, 132.8 (C, Ar), 130.8 (2CH, Ar), 129.7 (CH, Ar), 129.4 (2CH, Ar), 129.2 (2CH, Ar), $124.5(2 \mathrm{CH}, \mathrm{Ar}), 59.8\left(\mathrm{NCH}_{2}\right), 38.5\left(\mathrm{NCH}_{3}\right), 21.6\left(\mathrm{CH}_{3}, p\right.$-tolyl). IR (KBr): $v_{\text {máx }} 3436$, $3031,2920,1491,1455,1426,1321,1084,1053,811,748,709,566 .[\alpha]_{\mathbf{D}}^{25}-170.8(c$

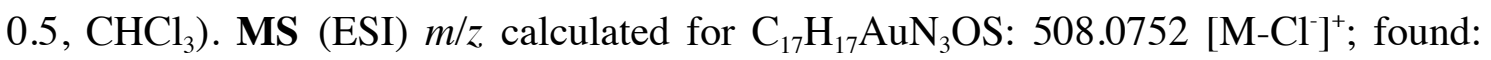
508.0756. mp: decomposed before melting.

\section{Preparation of compound 5ab}

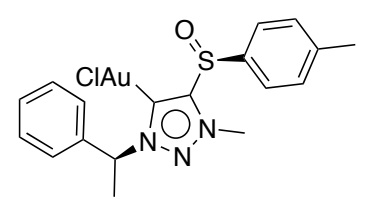

Following the general procedure a mixture of $\mathbf{4 a b}(300 \mathrm{mg}, 0.73 \mathrm{mmol}, 1.00$ equiv), $\mathrm{NMe}_{4} \mathrm{Cl}$ (119 mg, 1.09 mmol, 1.50 equiv) and $\mathrm{Ag}_{2} \mathrm{O}$ (126 mg, $0.54 \mathrm{mmol}, 0.75$ equiv) in $\mathrm{CH}_{3} \mathrm{CN}: \mathrm{CH}_{2} \mathrm{Cl}_{2}$ 1:10 (35 mL) was stirred under argon at $\mathrm{rt}$ overnight. [AuCl$\left(\mathrm{SMe}_{2}\right)$ ] 
(213 mg, $0.73 \mathrm{mmol}, 1.00$ equiv) was added and the reaction was stirred for two more hours. The resulting residue was purified $\left(\mathrm{SiO}_{2}, \mathrm{MeOH}: \mathrm{CH}_{2} \mathrm{Cl}_{2} 2 \%\right)$ to yield $\mathbf{6 a b}$ as a white solid (354 mg, 87\%).

${ }^{1}$ H NMR (400 MHz, $\mathrm{CDCl}_{3}$ ): $\delta 7.69$ (d, $J=8.3 \mathrm{~Hz}, 2 \mathrm{H}, \mathrm{Ar} p$-tolyl), 7.48 (m, 2H, Ar), $7.36(\mathrm{~m}, 5 \mathrm{H}, 3 \mathrm{H} \mathrm{Ar}+2 \mathrm{H}$ Ar p-tolyl), 6.23 (q, $J=7.1 \mathrm{~Hz}, 1 \mathrm{H}, \mathrm{CH}), 4.14\left(\mathrm{~s}, 3 \mathrm{H}, \mathrm{NCH}_{3}\right)$, 2.42 (s, 3H, $\mathrm{CH}_{3}, p$-tolyl), 1.99 (d, $\left.J=7.1 \mathrm{~Hz}, 3 \mathrm{H}, \mathrm{CH}_{3}\right) .{ }^{13} \mathbf{C} \mathbf{N M R}\left(100 \mathrm{MHz}, \mathrm{CDCl}_{3}\right)$ : ठ $160.2\left(\mathrm{C}, \mathrm{N}_{3} \mathrm{C}=\mathrm{CAu}\right), 146.4\left(\mathrm{C}, \mathrm{N}_{3} \mathrm{C}=\mathrm{CAu}\right), 143.0$ (C, Ar), 137.7 (C, Ar), 136.9 (C, Ar), 130.7 (2CH, Ar), 129.5 (CH, Ar), 129.2 (2CH, Ar), 127.2 (2CH, Ar), $124.5(2 \mathrm{CH}$, Ar), $66.8(\mathrm{CH}), 38.6\left(\mathrm{NCH}_{3}\right), 21.6\left(\mathrm{CH}_{3}\right.$, p-tolyl), $20.7\left(\mathrm{CH}_{3}\right)$. IR $(\mathbf{K B r}): \boldsymbol{v}_{\text {máx }} 3032$, $2982,1594,1492,1451,1425,1381,1333,1237,1083,1049,813,757,709,540 .[\alpha]_{\mathbf{D}}^{25}$ - $246.4\left(c \text { 1.0, } \mathrm{CHCl}_{3} \text { ). MS (ESI) } \mathrm{m} / z \text { calculated for } \mathrm{C}_{18} \mathrm{H}_{19} \mathrm{AuN}_{3} \mathrm{OS}: 522.0909 \text { [M-Cl}\right]^{+}$; found: 522.0929. mp: decomposed before melting.

\section{Preparation of compound 5ac}

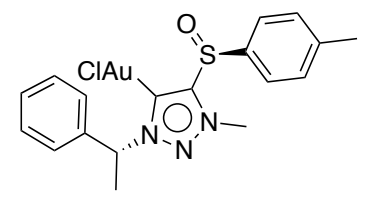

Following the general procedure a mixture of $\mathbf{4 a c}$ (206 $\mathrm{mg}, 0.50 \mathrm{mmol}, 1.00$ equiv), $\mathrm{NMe}_{4} \mathrm{Cl}$ (82 mg, $0.75 \mathrm{mmol}, 1.50$ equiv) and $\mathrm{Ag}_{2} \mathrm{O}(87 \mathrm{mg}, 0.37 \mathrm{mmol}, 0.75$ equiv) in $\mathrm{CH}_{3} \mathrm{CN}: \mathrm{CH}_{2} \mathrm{Cl}_{2}$ 1:1 (24 mL) was stirred under argon at rt overnight. [ $\left.\mathrm{AuCl}\left(\mathrm{SMe}_{2}\right)\right](147$ $\mathrm{mg}, 0.50 \mathrm{mmol}, 1.00$ equiv) was added and the reaction was stirred for two more hours. The resulting residue was purified $\left(\mathrm{SiO}_{2}, \mathrm{MeOH}: \mathrm{CH}_{2} \mathrm{Cl}_{2} 1 \%\right)$ to yield 5ac as a white solid (220 mg, $79 \%)$.

${ }^{1}$ H NMR (400 MHz, $\left.\mathrm{CDCl}_{3}\right): \delta 7.63$ (d, $J=8.6 \mathrm{~Hz}, 2 \mathrm{H}, \mathrm{Ar} p$-tolyl), 7.48 (m, 2H, Ar), $7.35\left(\mathrm{~m}, 5 \mathrm{H}, 3 \mathrm{H} \mathrm{Ar}+2 \mathrm{H}\right.$ Ar $p$-tolyl), $6.28(\mathrm{q}, J=7.1 \mathrm{~Hz}, 1 \mathrm{H}, \mathrm{CH}), 4.15\left(\mathrm{~s}, 3 \mathrm{H}, \mathrm{NCH}_{3}\right)$, 2.39 (s, 3H, $\mathrm{CH}_{3}, p$-tolyl), 2.02 (d, $\left.J=7.1 \mathrm{~Hz}, 3 \mathrm{H}, \mathrm{CH}_{3}\right) .{ }^{13} \mathbf{C} \mathbf{N M R}\left(100 \mathrm{MHz}, \mathrm{CDCl}_{3}\right)$ :

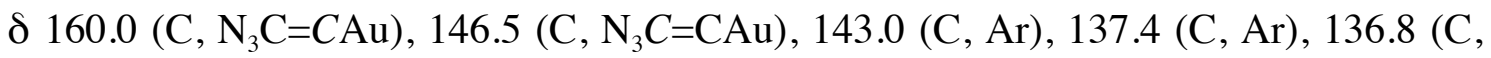
$\mathrm{Ar}), 130.7$ (2CH, Ar), 129.5 (CH, Ar), 129.2 (2CH, Ar), 127.5 (2CH, Ar), 124.5 (2CH, Ar), $66.7(\mathrm{CH}), 38.6\left(\mathrm{NCH}_{3}\right), 21.6\left(\mathrm{CH}_{3}\right.$, p-tolyl), $21.0\left(\mathrm{CH}_{3}\right)$. IR (KBr): $\boldsymbol{v}_{\text {máx }} 3032$, 
$2982,1593,1491,1456,1424,1381,1326,1083,1052,809,754,709,562,542 .[\alpha]_{D}^{25}-$

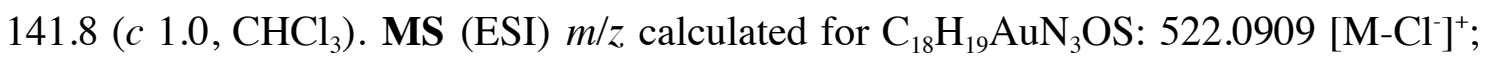
found: 522.0899. mp: decomposed before melting.

\section{Preparation of compound 5ba}

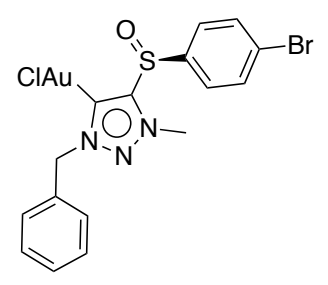

Following the general procedure a mixture of $4 \mathbf{b a}(70 \mathrm{mg}, 0.15 \mathrm{mmol}, 1.00$ equiv), $\mathrm{NMe}_{4} \mathrm{Cl}$ (37 mg, $0.34 \mathrm{mmol}, 2.25$ equiv) and $\mathrm{Ag}_{2} \mathrm{O}$ (39 mg, $0.17 \mathrm{mmol}, 1.13$ equiv) in $\mathrm{CH}_{3} \mathrm{CN}: \mathrm{CH}_{2} \mathrm{Cl}_{2}$ 1:10 (5.5 mL) was stirred under argon at $\mathrm{rt}$ for 3 days. [ $\mathrm{AuCl}\left(\mathrm{SMe}_{2}\right)$ ] (45 mg, $0.15 \mathrm{mmol}, 1.00$ equiv) was added and the reaction was stirred for two more hours. The resulting residue was purified $\left(\mathrm{SiO}_{2}\right.$, hexane:EtAcO 4:6) to yield $\mathbf{5 b a}$ as a white solid (61 mg, $69 \%)$.

${ }^{1} \mathbf{H}$ NMR $\left(400 \mathrm{MHz}, \mathrm{CDCl}_{3}\right): \delta 7.70\left(\mathrm{~s}, 4 \mathrm{H}, \mathrm{Ar} p-\mathrm{BrC}_{6} \mathrm{H}_{4}\right), 7.52(\mathrm{~m}, 2 \mathrm{H}, \mathrm{Ar}), 7.39(\mathrm{~m}$, $3 \mathrm{H}, \mathrm{Ar}), 5.68\left(\mathrm{~d}, J=14.0 \mathrm{~Hz}, 1 \mathrm{H}, \mathrm{NCH}_{2}\right), 5.62$ (d, $\left.J=14.0 \mathrm{~Hz}, 1 \mathrm{H}, \mathrm{NCH}_{2}\right), 4.15(\mathrm{~s}, 3 \mathrm{H}$, $\left.\mathrm{NCH}_{3}\right) .{ }^{13} \mathrm{C}$ NMR $\left(100 \mathrm{MHz}, \mathrm{CDCl}_{3}\right): \delta 160.9\left(\mathrm{C}, \mathrm{N}_{3} \mathrm{C}=C \mathrm{Au}\right), 145.9\left(\mathrm{C}, \mathrm{N}_{3} C=\mathrm{CAu}\right)$, 139.4 (C, Ar), 133.3 (2CH, Ar), 132.6 (C, Ar), 129.8 (CH, Ar), 129.4 (2CH, Ar), 129.2 (2CH, Ar), $127.1(\mathrm{C}, \mathrm{Ar}), 126.1(2 \mathrm{CH}, \mathrm{Ar}), 60.0\left(\mathrm{NCH}_{2}\right), 38.6\left(\mathrm{NCH}_{3}\right)$. IR $(\mathbf{K B r}): v_{\text {máx }}$ 3436, 3031, 1568, 1496, 1469, 1455, 1385, 1322, 1086, 1054, 1006, 817, 722, 709. $[\alpha]_{\mathbf{D}}^{25} \& 19.6\left(c \quad 0.8, \mathrm{CHCl}_{3}\right)$. MS (ESI) $m / z$ calculated for $\mathrm{C}_{16} \mathrm{H}_{18} \mathrm{AuBrClN}_{4} \mathrm{OS}$ : $624.9733\left[\mathrm{M}+\mathrm{NH}_{4}\right]^{+}$; found 624.9776. mp: decomposed before melting.

\section{Preparation of compound 5ca}

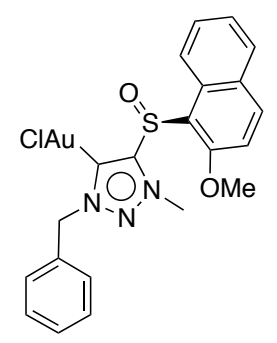


Following the general procedure a mixture of $4 \mathbf{c a}(250 \mathrm{mg}, 0.54 \mathrm{mmol}, 1.00$ equiv), $\mathrm{NMe}_{4} \mathrm{Cl}$ (88 mg, $0.81 \mathrm{mmol}, 1.50$ equiv) and $\mathrm{Ag}_{2} \mathrm{O}(93 \mathrm{mg}, 0.40 \mathrm{mmol}, 0.75$ equiv) in $\mathrm{CH}_{3} \mathrm{CN}: \mathrm{CH}_{2} \mathrm{Cl}_{2}$ 1:1 (29 mL) was stirred under argon at rt overnight. [ $\left.\mathrm{AuCl}\left(\mathrm{SMe}_{2}\right)\right](158$ $\mathrm{mg}, 0.54 \mathrm{mmol}, 1.00$ equiv) was added and the reaction was stirred for two more hours. The resulting residue was purified $\left(\mathrm{SiO}_{2}, \mathrm{Hex} / \mathrm{AcOEt} 3: 7\right)$ to yield 5ca as a white solid (287 mg, $87 \%)$.

${ }^{1} \mathbf{H}$ NMR $\left(400 \mathrm{MHz}, \mathrm{CDCl}_{3}\right): \delta 8.56(\mathrm{dd}, J=8.7 \mathrm{~Hz}, 0.9 \mathrm{~Hz}, 1 \mathrm{H}, \mathrm{Ar}), 8.11(\mathrm{~d}, J=9.1$ $\mathrm{Hz}, 1 \mathrm{H}, \mathrm{Ar}), 7.84(\mathrm{~d}, J=8.1 \mathrm{~Hz}, 1 \mathrm{H}, \mathrm{Ar}), 7.60$ (ddd, $J=8.5 \mathrm{~Hz}, 6.9 \mathrm{~Hz}, 1.3 \mathrm{~Hz}, 1 \mathrm{H}$, Ar), 7.44 (ddd, $J=8.0 \mathrm{~Hz}, 6.9 \mathrm{~Hz}, 1.0 \mathrm{~Hz}, 1 \mathrm{H}, \mathrm{Ar}), 7.40$ (m, 2H, Ar), 7.33 (m, 4H, Ar), $5.50\left(\mathrm{~d}, J=14.1 \mathrm{~Hz}, 1 \mathrm{H}, \mathrm{NCH}_{2}\right), 5.45\left(\mathrm{~d}, J=14.1 \mathrm{~Hz}, 1 \mathrm{H}, \mathrm{NCH}_{2}\right), 4.53\left(\mathrm{~s}, 3 \mathrm{H}, \mathrm{NCH}_{3}\right)$, 4.00 (s, 3H, 2-OCH 3 naph). ${ }^{13} \mathrm{C}$ NMR (100 MHZ, $\left.\mathrm{CDCl}_{3}\right): \delta 159.0\left(\mathrm{C}, \mathrm{N}_{3} \mathrm{C}=C \mathrm{Au}\right)$, $158.1(\mathrm{C}, \mathrm{Ar}), 146.3\left(\mathrm{C}, \mathrm{N}_{3} \mathrm{C}=\mathrm{CAu}\right), 137.2(\mathrm{CH}, \mathrm{Ar}), 133.1(\mathrm{C}, \mathrm{Ar}), 132.0(\mathrm{C}, \mathrm{Ar})$, 129.5 (C, Ar), 129.4 (CH, Ar), 129.4 (CH, Ar), 129.3 (CH, Ar), 129.1 (2CH, Ar), 128.9 (2CH, Ar), 125.1 ( $\mathrm{CH}, \mathrm{Ar}), 122.5(\mathrm{CH}, \mathrm{Ar}), 120.7$ (C, Ar), $113.1(\mathrm{CH}, \mathrm{Ar}), 59.7$ $\left(\mathrm{NCH}_{2}\right), 57.0$ (2-OCH $\mathrm{OCH}_{3}$ naph), $39.2\left(\mathrm{NCH}_{3}\right)$. IR (KBr): $\boldsymbol{v}_{\text {máx }}$ 3436, 2943, 1619, 1592, $1507,1430,1274,1251,1057,814,747,708,650 . .[\alpha]_{\mathbf{D}}^{25}-3.3\left(c 0.6, \mathrm{CHCl}_{3}\right) . \mathbf{M S}$

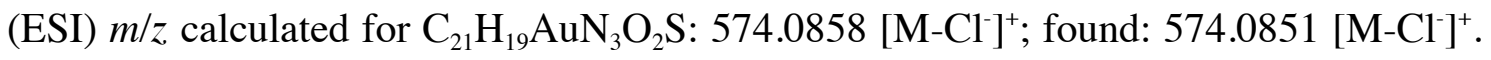
mp: decomposed before melting.

\section{Preparation of compound 5cd}

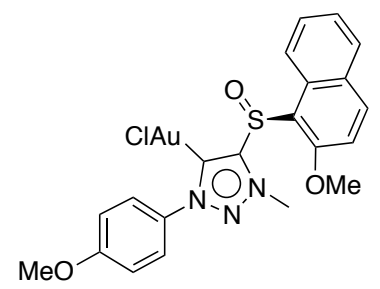

Following the general procedure a mixture of $4 \mathbf{c d}$ (250 $\mathrm{mg}, 0.52 \mathrm{mmol}, 1.00$ equiv), $\mathrm{NMe}_{4} \mathrm{Cl}$ (85 mg, $0.78 \mathrm{mmol}, 1.50$ equiv) and $\mathrm{Ag}_{2} \mathrm{O}$ (90 mg, $0.39 \mathrm{mmol}, 0.75$ equiv) in $\mathrm{CH}_{3} \mathrm{CN}: \mathrm{CH}_{2} \mathrm{Cl}_{2}$ 1:1 (29 mL) was stirred under argon at rt for overnight. [ $\mathrm{AuCl}\left(\mathrm{SMe}_{2}\right)$ ] (153 mg, $0.52 \mathrm{mmol}, 1.00$ equiv) was added and the reaction was stirred for two more hours. The resulting residue was purified $\left(\mathrm{SiO}_{2}, \mathrm{Hex} / \mathrm{AcOEt} 3: 7\right)$ to yield $\mathbf{5 c d}$ as a white solid (297 mg, $91 \%)$. 
${ }^{1}$ H NMR (400 MHz, $\left.\mathrm{CDCl}_{3}\right): \delta 8.69(\mathrm{dd}, J=8.4 \mathrm{~Hz}, 0.8 \mathrm{~Hz}, 1 \mathrm{H}, \mathrm{Ar}), 8.13(\mathrm{~d}, J=9.2$ $\mathrm{Hz}, 1 \mathrm{H}, \mathrm{Ar}), 7.84\left(\mathrm{~m}, 3 \mathrm{H}, 1 \mathrm{H} \mathrm{Ar}+2 \mathrm{H} p-\mathrm{OCH}_{3} \mathrm{C}_{6} \mathrm{H}_{4}\right), 7.66(\mathrm{ddd}, J=8.5 \mathrm{~Hz}, 7.0 \mathrm{~Hz}, 1.3$ $\mathrm{Hz}, 1 \mathrm{H}, \mathrm{Ar}$ ), 7.47 (ddd, $J=8.0 \mathrm{~Hz}, 7.0 \mathrm{~Hz}, 1.0 \mathrm{~Hz}, 1 \mathrm{H}, \mathrm{Ar}$ ), 7.32 (d, $J=9.2 \mathrm{~Hz}, 1 \mathrm{H}$, Ar), $6.91\left(\mathrm{~d}, J=9.2 \mathrm{~Hz}, 2 \mathrm{H}, \mathrm{Ar} p-\mathrm{OCH}_{3} \mathrm{C}_{6} \mathrm{H}_{4}\right), 4.68$ (s, 3H, $\left.\mathrm{NCH}_{3}\right), 4.09$ (s, 3H, 2$\mathrm{OCH}_{3}$ naph), 3.81 (s, $\left.3 \mathrm{H}, p-\mathrm{OCH}_{3} \mathrm{C}_{6} \mathrm{H}_{4}\right) .{ }^{13} \mathrm{C} \mathrm{NMR}\left(100 \mathrm{MHz}, \mathrm{CDCl}_{3}\right): \delta 161.2(\mathrm{C}, \mathrm{Ar})$, 158.0 (C, Ar), 157.5 (C, $\left.\mathrm{N}_{3} \mathrm{C}=\mathrm{CAu}\right), 146.3\left(\mathrm{C}, \mathrm{N}_{3} \mathrm{C}=\mathrm{CAu}\right), 137.2$ (CH, Ar), 132.1 (C, Ar), 131.8 (C, Ar), 129.5 (CH, Ar), 129.3 (CH, Ar), 129.4 (C, Ar), 125.2 (CH, Ar), 125.1 (2CH, Ar), 122.7 (CH, Ar), 121.1 (C, Ar), 114.6 (2CH, Ar), 113.1 (CH, Ar), 57.1 (2-OCH $\mathrm{H}_{3}$ naph), $55.8\left(p-\mathrm{OCH}_{3} \mathrm{C}_{6} \mathrm{H}_{4}\right), 39.2\left(\mathrm{NCH}_{3}\right)$. IR (KBr): $\boldsymbol{v}_{\text {máx }} 3435,2936,2838$, $1592,1509,1467,1274,1253,1175,1152,1065,835,809,741 .[\alpha]_{\mathbf{D}}^{25}-10.0(c 0.5$, $\mathrm{CHCl}_{3}$ ). MS (ESI) $\mathrm{m} / z$ calculated for $\mathrm{C}_{21} \mathrm{H}_{19} \mathrm{AuN}_{3} \mathrm{O}_{3} \mathrm{~S}: 590.0807$ [M-Cl$]^{+}$; found: 590.0808. mp: decomposed before melting.

\section{Preparation of compound 5ce}

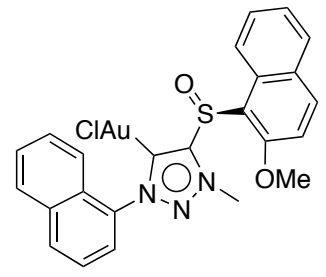

Following the general procedure a mixture of 4 ce $(300 \mathrm{mg}, 0.60 \mathrm{mmol}, 1.00$ equiv), $\mathrm{NMe}_{4} \mathrm{Cl}$ (98 mg, $0.9 \mathrm{mmol}, 1.50$ equiv) and $\mathrm{Ag}_{2} \mathrm{O}$ (104 mg, $0.45 \mathrm{mmol}, 0.75$ equiv) in $\mathrm{CH}_{3} \mathrm{CN}: \mathrm{CH}_{2} \mathrm{Cl}_{2}$ 1:1 (34 mL) was stirred under argon at rt overnight. [ $\left.\mathrm{AuCl}\left(\mathrm{SMe}_{2}\right)\right](176$ $\mathrm{mg}, 0.6 \mathrm{mmol}, 1.00$ equiv) was added and the reaction was stirred for two more hours. The resulting residue was purified $\left(\mathrm{SiO}_{2}\right.$, Hex/AcOEt 3:7) to yield 5ce as a white solid (339 mg, $87 \%)$.

${ }^{1} \mathbf{H}$ NMR $\left(400 \mathrm{MHz}, \mathrm{CDCl}_{3}\right): \delta 8.68(\mathrm{~d}, J=8.6 \mathrm{~Hz}, 1 \mathrm{H}, \mathrm{Ar}), 8.14(\mathrm{~d}, J=9.1 \mathrm{~Hz}, 1 \mathrm{H}$, Ar), $8.00(\mathrm{~d}, J=8.2 \mathrm{~Hz}, 1 \mathrm{H}, \mathrm{Ar}), 7.90(\mathrm{~d}, J=12.2 \mathrm{~Hz}, 1 \mathrm{H}, \mathrm{Ar}), 7.88(\mathrm{~d}, J=12.2 \mathrm{~Hz}$, $1 \mathrm{H}, \mathrm{Ar}), 7.68$ (t, $J=7.8 \mathrm{~Hz}, 1 \mathrm{H}, \mathrm{Ar}), 7.60$ (d, $J=7.4 \mathrm{~Hz}, 1 \mathrm{H}, \mathrm{Ar}), 7.48$ (m, 4H, Ar), $7.35(\mathrm{~d}, J=9.2 \mathrm{~Hz}, 1 \mathrm{H}, \mathrm{Ar}), 7.11$ (d, $J=8.5 \mathrm{~Hz}, 1 \mathrm{H}, \mathrm{Ar}), 4.75$ (s, 3H, $\left.\mathrm{NCH}_{3}\right), 4.14$ (s, $3 \mathrm{H}, 2-\mathrm{OCH}_{3}$ naph). ${ }^{13} \mathrm{C}$ NMR (100 MHz, $\left.\mathrm{CDCl}_{3}\right): \delta 162.3\left(\mathrm{C}, \mathrm{N}_{3} \mathrm{C}=C \mathrm{Au}\right), 158.3(\mathrm{C}$, Ar), $146.2\left(\mathrm{C}, \mathrm{N}_{3} \mathrm{C}=\mathrm{CAu}\right), 137.4(\mathrm{CH}, \mathrm{Ar}), 135.3$ (C, Ar), 134.1 (C, Ar), 132.1 (C, Ar), 
$131.8(\mathrm{CH}, \mathrm{Ar}), 129.6$ (C, Ar), $129.5(\mathrm{CH}, \mathrm{Ar}), 129.4(\mathrm{CH}, \mathrm{Ar}), 128.6(\mathrm{CH}, \mathrm{Ar}), 128.3$ (CH, Ar), 128.2 (C, Ar), 127.4 (CH, Ar), 125.1 (CH, Ar), 124.9 (CH, Ar), 124.9 (CH, Ar), 122.5 (CH, Ar) $121.4(\mathrm{CH}, \mathrm{Ar}) 121.1$ (C, Ar), 113.2 (CH, Ar), 57.1 (2-OCH $\mathrm{CH}_{3}$ nap), $39.6\left(\mathrm{NCH}_{3}\right)$. IR (KBr): v máx $^{2436, ~ 3056, ~ 2987, ~ 1620, ~ 1592, ~ 1508, ~ 1468, ~ 1274, ~ 1251, ~}$

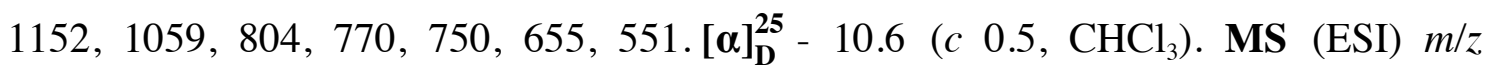

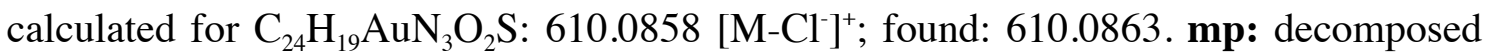
before melting.

\section{Preparation of compound 9b}

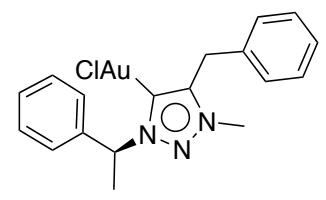

Following the general procedure a mixture of $\mathbf{1 2 b}(200 \mathrm{mg}, 0.55 \mathrm{mmol}, 1.00$ equiv), $\mathrm{NMe}_{4} \mathrm{Cl}$ (90 mg, $0.82 \mathrm{mmol}, 1.50$ equiv) and $\mathrm{Ag}_{2} \mathrm{O}$ (95 mg, $0.42 \mathrm{mmol}, 0.75$ equiv) in $\mathrm{CH}_{3} \mathrm{CN}: \mathrm{CH}_{2} \mathrm{Cl}_{2}$ 1:10 (33 mL) was stirred under argon at $\mathrm{rt}$ overnight. [AuCl$\left(\mathrm{SMe}_{2}\right)$ ] (160 mg, $0.55 \mathrm{mmol}, 1.00$ equiv) was added and the reaction was stirred for two more hours. The resulting residue was purified $\left(\mathrm{SiO}_{2}, \mathrm{CH}_{2} \mathrm{Cl}_{2}\right)$ to yield $\mathbf{9 b}$ as a white solid (213 mg, $76 \%)$.

${ }^{1} \mathbf{H}$ NMR $\left(400 \mathrm{MHz}, \mathrm{CDCl}_{3}\right): \delta 7.50(\mathrm{~d}, J=7.7 \mathrm{~Hz}, 2 \mathrm{H}, \mathrm{Ar}), 7.28(\mathrm{~m}, 8 \mathrm{H}, \mathrm{Ar}), 6.26$ (q, $J=7.2 \mathrm{~Hz}, 1 \mathrm{H}, \mathrm{CH}), 4.17\left(\mathrm{~d}, J=16.1 \mathrm{~Hz}, 1 \mathrm{H}, \mathrm{NCH}_{2}\right), 4.08\left(\mathrm{~d}, J=16.1 \mathrm{~Hz}, 1 \mathrm{H}, \mathrm{NCH}_{2}\right)$, $3.85\left(\mathrm{~s}, 3 \mathrm{H}, \mathrm{NCH}_{3}\right), 1.98\left(\mathrm{~d}, J=7.2 \mathrm{~Hz}, 3 \mathrm{H}, \mathrm{CH}_{3}\right) .{ }^{13} \mathbf{C} \mathbf{N M R}\left(125 \mathrm{MHz}, \mathrm{CDCl}_{3}\right): \delta$ $157.7\left(\mathrm{C}, \mathrm{N}_{3} \mathrm{C}=\mathrm{CAu}\right), 145.5\left(\mathrm{C}, \mathrm{N}_{3} \mathrm{C}=\mathrm{CAu}\right), 138.5(\mathrm{C}, \mathrm{Ar}), 135.1(\mathrm{C}, \mathrm{Ar}), 129.2(2 \mathrm{CH}$, $\mathrm{Ar}), 129.0(3 \mathrm{CH}, \mathrm{Ar}), 128.5(2 \mathrm{CH}, \mathrm{Ar}), 127.6(\mathrm{CH}, \mathrm{Ar}), 127.3(2 \mathrm{CH}, \mathrm{Ar}), 65.4(\mathrm{CH})$, $37.3\left(\mathrm{NCH}_{3}\right), 31.3\left(\mathrm{NCH}_{2}\right), 20.9\left(\mathrm{CH}_{3}\right)$. IR (KBr): $\boldsymbol{v}_{\text {máx }}$ 3435, 3028, 1494, 1455, 1321,

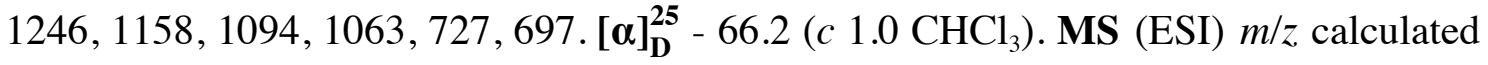
for $\mathrm{C}_{18} \mathrm{H}_{19} \mathrm{AuN}_{3}$ : 474.1239 [M-Cl$]^{+}$; found: 474.1236. mp: decomposed before melting.

\section{Preparation of compound 9c}

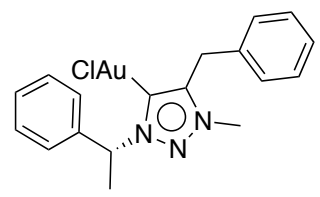


Following the general procedure a mixture of $12 \mathrm{c}$ (200 $\mathrm{mg}, 0.55 \mathrm{mmol}, 1.00$ equiv), $\mathrm{NMe}_{4} \mathrm{Cl}$ (90 mg, $0.82 \mathrm{mmol}, 1.50$ equiv) and $\mathrm{Ag}_{2} \mathrm{O}(95 \mathrm{mg}, 0.42 \mathrm{mmol}, 0.75$ equiv) in $\mathrm{CH}_{3} \mathrm{CN}: \mathrm{CH}_{2} \mathrm{Cl}_{2}$ 1:10 (33 mL) was stirred under argon at $\mathrm{rt}$ overnight. [ $\mathrm{AuCl}\left(\mathrm{SMe}_{2}\right)$ ] (160 mg, $0.55 \mathrm{mmol}, 1.00$ equiv) was added and the reaction was stirred for two more hours. The resulting residue was purified $\left(\mathrm{SiO}_{2}, \mathrm{CH}_{2} \mathrm{Cl}_{2}\right)$ to yield $9 \mathrm{c}$ as a white solid (173 mg, $62 \%)$.

${ }^{1} \mathbf{H}$ NMR $\left(400 \mathrm{MHz}, \mathrm{CDCl}_{3}\right): \delta 7.50(\mathrm{~d}, J=7.7 \mathrm{~Hz}, 2 \mathrm{H}, \mathrm{Ar}), 7.28(\mathrm{~m}, 8 \mathrm{H}, \mathrm{Ar}), 6.26$ (q, $J=7.2 \mathrm{~Hz}, 1 \mathrm{H}, \mathrm{CH}), 4.17\left(\mathrm{~d}, J=16.1 \mathrm{~Hz}, 1 \mathrm{H}, \mathrm{NCH}_{2}\right), 4.08\left(\mathrm{~d}, J=16.1 \mathrm{~Hz}, 1 \mathrm{H}, \mathrm{NCH}_{2}\right)$, $3.85\left(\mathrm{~s}, 3 \mathrm{H}, \mathrm{NCH}_{3}\right), 1.98\left(\mathrm{~d}, J=7.2 \mathrm{~Hz}, 3 \mathrm{H}, \mathrm{CH}_{3}\right) \cdot{ }^{13} \mathbf{C} \mathbf{N M R}\left(125 \mathrm{MHz}, \mathrm{CDCl}_{3}\right): \delta$ $157.8\left(\mathrm{C}, \mathrm{N}_{3} \mathrm{C}=\mathrm{CAu}\right), 145.5\left(\mathrm{C}, \mathrm{N}_{3} C=\mathrm{CAu}\right), 138.5$ (C, Ar), $135.0(\mathrm{C}, \mathrm{Ar}), 129.2(2 \mathrm{CH}$, Ar), 129.1 (CH, Ar), 129.0 (2CH, Ar), 128.6 (2CH, Ar), $127.6(\mathrm{CH}, \mathrm{Ar}), 127.3(2 \mathrm{CH}$, Ar), $65.4(\mathrm{CH}), 37.3\left(\mathrm{NCH}_{3}\right), 31.3\left(\mathrm{NCH}_{2}\right), 20.9\left(\mathrm{CH}_{3}\right)$. IR (KBr): $\boldsymbol{v}_{\text {máx }} 3435,3028$, $1495,1455,1321,1247,1063,727,697 .[\alpha]_{\mathbf{D}}^{25}+65.5\left(c 1.0, \mathrm{CHCl}_{3}\right) . \mathbf{M S}(\mathrm{ESI}) \mathrm{m} / \mathrm{z}$ calculated for $\mathrm{C}_{18} \mathrm{H}_{19} \mathrm{Au} \mathrm{N} \mathrm{N}_{3}$ : 474.1239 [M-Cl$]^{+}$; found: 474.1249. mp: decomposed before melting.

\section{Preparation of enynes}

Enynes 6, 13, 16, 21, 23, 25 were prepared from dimethyl malonate following the general methods previously described. ${ }^{5}$

Enyne 18 was prepared from 2-buten-1-ol following the method described in literature. ${ }^{6}$

The structure of cycloisomerization products $7^{7}, \mathbf{1 4}^{8}, \mathbf{1 5}^{8}, \mathbf{1 7}^{9}, \mathbf{1 9}^{\mathbf{1 0}}$ was confirmed by ${ }^{1} \mathrm{H}$ NMR according to literature data.

\footnotetext{
${ }^{5}$ Buisine, O.; Aubert, C.; Malacria, M. Chem. Eur. J. 2001, 7, 3517.

${ }^{6}$ Hoye, T. R.; Baire, B.; Niu, D.; Willoughby, P. H.; Woods, B. P. Nature 2012, 490, 208.

${ }^{7}$ Inagaki, F.; Matsumoto, C.; Okada, Y.; Maruyama, N.; Mukai, C. Angew. Chem. Int. Ed. 2015, 54,818 .

${ }^{8}$ Nieto-Oberhuber, C.; Muñoz, M. P.; López, S.; Jiménez-Núñez, E.; Nevado, C.; HerreroGómez, E.; Raducan, M.; Echavarren, A. M. Chem. Eur. J. 2006, 12, 1677.

${ }^{9}$ Gryparis, C.; Efe, C.; Raptis, C.; Lykakis, I. N.; Stratakis, M. Org. Lett. 2012, 14, 2956.

${ }^{10}$ Hart-Cooper, W. M.; Clary, K. N.; Toste, F. D.; Bergman, R. G.; Raymond, K. N. J. Am. Chem. Soc. 2012, 134, 17873.
} 


\section{Synthesis of enynes 21}

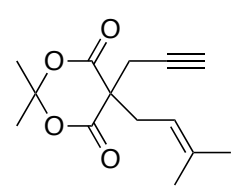

2,2-dimethyl-1,3-dioxane-4,6-dione ( $1451 \mathrm{mg}, 10 \mathrm{mmol}, 1.00$ equiv) were dissolved in $15 \mathrm{~mL}$ of anhydrous THF and added over a suspension of $\mathrm{NaH}(60 \%$ dispersion in mineral oil) (443 mg, $11.07 \mathrm{mmol}, 1.1$ equiv) in anhydrous $\mathrm{THF}(6 \mathrm{~mL})$ at $0{ }^{\circ} \mathrm{C}$. The mixture was stirred at $\mathrm{rt}$ for $30 \mathrm{~min}$. 3,3-dimethylallyl bromide (1.6 mL, $11.07 \mathrm{mmol}$, 1.10 equiv) was added at $0{ }^{\circ} \mathrm{C}$. After being stirred at $\mathrm{rt}$ overnight, the reaction was quenched with an aqueous solution of $\mathrm{HCl} 0.1 \mathrm{M}$ to reach an acid $\mathrm{pH}$. The mixture was extracted with $\mathrm{Et}_{2} \mathrm{O}$ (three times) and the combined organic layers were washed with brine, dried over $\mathrm{MgSO}_{4}$ and filtered. The volatiles were evaporated under vacuum and the residue was purified by a short pad of $\mathrm{SiO}_{2}(\mathrm{Hex}$ :AcOEt 5:1) obtaining a white solid (782 mg, 37\%).

The allylated intermediate (782 mg, $3.68 \mathrm{mmol}, 1.00$ equiv) was dissolved in anhydrous DMF (5 mL) and added over a suspension of $\mathrm{NaH}$ (60\% dispersion in mineral oil) (177 $\mathrm{mg}, 4.43 \mathrm{mmol}, 1.20$ equiv) in anhydrous DMF $(5 \mathrm{~mL})$ at $0{ }^{\circ} \mathrm{C}$. The mixture was stirred at $\mathrm{rt}$ for $30 \mathrm{~min}$ before propargyl bromide $(0.40 \mathrm{~mL}, 3.68 \mathrm{mmol}, 1.00$ equiv) was added at $0{ }^{\circ} \mathrm{C}$. After being stirred at $\mathrm{rt}$ overnight, the reaction was quenched with $\mathrm{H}_{2} \mathrm{O}$. An aqueous solution of $\mathrm{HCl} 0.1 \mathrm{M}$ is added dropwise to reach an acidic $\mathrm{pH}$. The mixture was extracted with $\mathrm{Et}_{2} \mathrm{O}$ (three times) and the combined organic layers were washed with brine, dried over $\mathrm{MgSO}_{4}$ and filtered. The volatiles were evaporated under vacuum and the residue was purified by a short pad of $\mathrm{SiO}_{2}$ (Hex:AcOEt 9:1) obtaining a white solid (430 mg, 47\%).

${ }^{1} \mathbf{H}$ NMR $\left(400 \mathrm{MHz}, \mathrm{CDCl}_{3}\right): \delta 5.02(\mathrm{t}, J=8.7 \mathrm{~Hz}, 1 \mathrm{H}, \mathrm{CH}), 2.89(\mathrm{~d}, J=2.6 \mathrm{~Hz}, 2 \mathrm{H}$, $\left.\mathrm{CH}_{2}\right), 2.67\left(\mathrm{~d}, J=8.0 \mathrm{~Hz}, 2 \mathrm{H}, \mathrm{CH}_{2}\right), 2.10(\mathrm{t}, J=2.6 \mathrm{~Hz}, 1 \mathrm{H}, \mathrm{CH}), 1.78\left(\mathrm{~s}, 3 \mathrm{H}, \mathrm{CH}_{3}\right)$, $1.69\left(\mathrm{~s}, 6 \mathrm{H}, 2 \mathrm{CH}_{3}\right), 1.60\left(\mathrm{~s}, 3 \mathrm{H}, \mathrm{CH}_{3}\right) .{ }^{13} \mathrm{C} \mathrm{NMR}\left(100 \mathrm{MHz}, \mathrm{CDCl}_{3}\right): \delta 168.3(2 \mathrm{C}=\mathrm{O})$, 139.1 (C), $116.4(\mathrm{CH}), 106.4(\mathrm{C}), 78.9(\mathrm{C}), 72.7(\mathrm{CH}), 54.7(\mathrm{C}), 38.6\left(\mathrm{CH}_{2}\right), 30.7\left(\mathrm{CH}_{3}\right)$, $29.2\left(\mathrm{CH}_{3}\right), 26.8\left(\mathrm{CH}_{2}\right), 26.0\left(\mathrm{CH}_{3}\right), 17.9\left(\mathrm{CH}_{3}\right)$. IR $(\mathbf{K B r}): \boldsymbol{v}_{\text {máx }} 3291,1772,1725$, 1341, 1265, 1104, 1051, 664. MS (ESI) $\mathrm{m} / z$ calculated for $\mathrm{C}_{14} \mathrm{H}_{18} \mathrm{NaO}_{4}: 273.1097$ $[\mathrm{M}+\mathrm{Na}]^{+}$; found: 273.1094 . mp: below $30{ }^{\circ} \mathrm{C}$. 


\section{General procedure for the cycloisomerization of enynes}

Gold carbene 5ab ( $0.01 \mathrm{mmol}, 0.03$ equiv) and $\operatorname{NaBArF}(0.01 \mathrm{mmol}, 0.03$ equiv) were stirred under $\mathrm{Ar}$ in $2 \mathrm{~mL}$ of anhydrous $\mathrm{CH}_{2} \mathrm{Cl}_{2}$ for $15 \mathrm{~min}$. A solution of enyne $(0.33$ mmol, 1.00 equiv) in $2 \mathrm{~mL}$ of anhydrous $\mathrm{CH}_{2} \mathrm{Cl}_{2}$ was added in one portion. The reaction mixture was stirred at rt until completion of the reaction. The crude was filtered through a short pad of Celite and $\mathrm{SiO}_{2}$. Solvent was removed under pressure. The crude was purified through a short pad of $\mathrm{SiO}_{2}$.

\section{Cycloisomerization of enyne 6}

Following the general procedure, gold carbene $5 \mathbf{a b}(6 \mathrm{mg}, 0.01 \mathrm{mmol}, 0.03$ equiv) and NaBArF ( $9 \mathrm{mg}, 0.01 \mathrm{mmol}, 0.03$ equiv) were dissolved in $2 \mathrm{~mL}$ anhydrous $\mathrm{CH}_{2} \mathrm{Cl}_{2}$ under argon for $15 \mathrm{~min}$. Enyne 6 (79 $\mathrm{mg}, 0.33 \mathrm{mmol}, 1.00$ equiv) was added dissolved in $2 \mathrm{~mL}$ of anhydrous $\mathrm{CH}_{2} \mathrm{Cl}_{2}$. After 10 min of stirring at $\mathrm{rt}$, the reaction was filtered through a short pad of Celite and $\mathrm{SiO}_{2}$. The crude was purified through a short pad of $\mathrm{SiO}_{2}$ (hexanes) to obtain 7 as colourless oil (79 mg, 100\%).

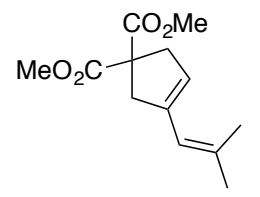

${ }^{1}$ H NMR (400 MHz, $\mathrm{CDCl}_{3}$ ): $\delta 5.72(\mathrm{~s}, 1 \mathrm{H}), 5.37$ (s, 1H), 3.72 (s, 6H), 3.18 (br d, $J$ $=1.7 \mathrm{~Hz}, 2 \mathrm{H}), 3.03(\mathrm{~s}, 2 \mathrm{H}), 1.81(\mathrm{~s}, 3 \mathrm{H}), 1.77(\mathrm{~s}, 3 \mathrm{H})$.

\section{Cycloisomerization of enyne 13}

Following the general procedure, gold carbene $5 \mathbf{a b}(6 \mathrm{mg}, 0.01 \mathrm{mmol}, 0.03$ equiv) and NaBArF (9 mg, $0.01 \mathrm{mmol}, 0.03$ equiv) were dissolved in $2 \mathrm{~mL}$ anhydrous $\mathrm{CH}_{2} \mathrm{Cl}_{2}$ under argon for $15 \mathrm{~min}$. Enyne 13 (94 mg, $0.33 \mathrm{mmol}, 1.00$ equiv) was added dissolved in $2 \mathrm{~mL}$ of anhydrous $\mathrm{CH}_{2} \mathrm{Cl}_{2}$. After $1 \mathrm{~h}$ of stirring at $\mathrm{rt}$, the reaction was filtered 
through a short pad of Celite and $\mathrm{SiO}_{2}$. The crude was purified through a short pad of $\mathrm{SiO}_{2}$ (hexane:AcOEt 9:1) to obtain $\mathbf{1 4}$ and $\mathbf{1 5}$ as a mixture (5:1) as white solid (84 $\mathrm{mg}$, $89 \%)$.

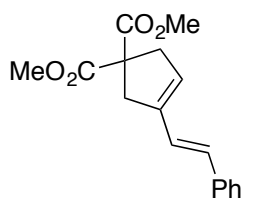

${ }^{1}$ H NMR $\left(300 \mathrm{MHz}, \mathrm{CDCl}_{3}\right): 7.23(\mathrm{~m}, 5 \mathrm{H}), 6.82(\mathrm{~d}, J=16.2 \mathrm{~Hz}, 1 \mathrm{H}), 6.37$ (d, $J=16.2$ $\mathrm{Hz}, 1 \mathrm{H}), 5.63$ (br s, 1H), 3.70 (s, 6H), 3.20 (s, 2H), 3.09 (s, 2H).

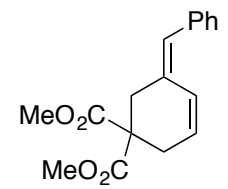

${ }^{1} \mathbf{H}$ NMR (300 MHz, $\mathrm{CDCl}_{3}$ ): 7.23 (m, 5H, overlapped signal), 6.53 (d, $J=10.1 \mathrm{~Hz}$, 1H), 6.27 (br s, 1H), $5.80(\mathrm{~m}, 1 \mathrm{H}), 3.66$ (s, 6H), 2.89 (br s, 2H), 2.69 (br s, 2H).

\section{Cycloisomerization of enyne 16}

Following the general procedure, gold carbene $\mathbf{5 a b}(6 \mathrm{mg}, 0.01 \mathrm{mmol}, 0.03$ equiv $)$ and $\mathrm{NaBArF}\left(9 \mathrm{mg}, 0.01 \mathrm{mmol}, 0.03\right.$ equiv) were dissolved in $2 \mathrm{~mL}$ anhydrous $\mathrm{CH}_{2} \mathrm{Cl}_{2}$ under argon for $15 \mathrm{~min}$. Enyne 16 (74 $\mathrm{mg}, 0.33 \mathrm{mmol}, 1.00$ equiv) was added dissolved in $2 \mathrm{~mL}$ of anhydrous $\mathrm{CH}_{2} \mathrm{Cl}_{2}$. After 60 min of stirring at $\mathrm{rt}$, the reaction was filtered through a short pad of Celite and $\mathrm{SiO}_{2}$. The crude was purified through a short pad of $\mathrm{SiO}_{2}$ (hexane:AcOEt 96:4) to obtain 17 as yellowish oil in 5:1 E/Z mixture (64 $\mathrm{mg}$, $86 \%)$.

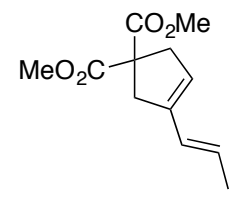

${ }^{1} \mathbf{H}$ NMR $\left(300 \mathrm{MHz}, \mathrm{CDCl}_{3}\right): \delta 6.18(\mathrm{br} \mathrm{d}, J=15.6 \mathrm{~Hz}, 1 \mathrm{H}), 5.60(\mathrm{dq}, J=15.6 \mathrm{~Hz}, 6.6$ $\mathrm{Hz}, 1 \mathrm{H}), 5.40$ (br m, 1H), 3.74 (s, 6H), 3.10 (br s, 2H), 3.07 (br s, 2H), 1.77 (br d, $J=$ $6.6 \mathrm{~Hz}, 3 \mathrm{H})$.

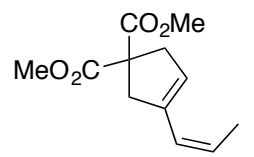


${ }^{1} \mathbf{H}$ NMR $\left(300 \mathrm{MHz}, \mathrm{CDCl}_{3}\right): \delta 5.93(\mathrm{dm}, J=11.6 \mathrm{~Hz}, 1 \mathrm{H}), 5.50(\mathrm{~m}, 2 \mathrm{H}$, overlapped signals), 3.75 (s, 6H), 3.28 (br s, 2H), 3.07 (s, 2H, overlapped signal), 1.83 (d, $J=7.5$ $\mathrm{Hz}, 3 \mathrm{H})$.

\section{Cycloisomerization of enyne 18}

Following the general procedure, gold carbene $\mathbf{5 a b}(14 \mathrm{mg}, 0.02 \mathrm{mmol}, 1.00$ equiv) and $\mathrm{NaBArF}$ (21 mg, $0.02 \mathrm{mmol}, 0.03$ equiv) were dissolved in $2.5 \mathrm{~mL}$ anhydrous $\mathrm{CH}_{2} \mathrm{Cl}_{2}$ under argon for $15 \mathrm{~min}$. Enyne 18 (100 mg, $0.81 \mathrm{mmol}, 1.00$ equiv) was added dissolved in $2.5 \mathrm{~mL}$ of anhydrous $\mathrm{CH}_{2} \mathrm{Cl}_{2}$. After 60 min of stirring at $\mathrm{rt}$, the reaction was filtered through a short pad of celite and $\mathrm{SiO}_{2}$. The crude was purified through a short pad of $\mathrm{SiO}_{2}\left(\mathrm{CH}_{2} \mathrm{Cl}_{2}\right)$ to obtain $\mathbf{1 9}$ and $\mathbf{2 0}$ as yellow oil in a mixture (3:1) (100 $\mathrm{mg}$, $100 \%)$.

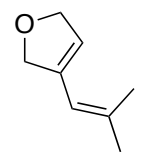

${ }^{1}$ H NMR $\left(400 \mathrm{MHz}, \mathrm{CDCl}_{3}\right): \delta 6.54(\mathrm{~d}, J=10.4 \mathrm{~Hz}, 1 \mathrm{H}, \mathrm{CH}), 5.73(\mathrm{br} \mathrm{d}, J=10.4 \mathrm{~Hz}$, $1 \mathrm{H}, \mathrm{CH}), 4.34\left(\mathrm{~s}, 2 \mathrm{H}, \mathrm{CH}_{2}\right), 4.18\left(\mathrm{~s}, 2 \mathrm{H}, \mathrm{CH}_{2}\right), 1.76\left(\mathrm{~s}, 3 \mathrm{H}, \mathrm{CH}_{3}\right), 1.69\left(\mathrm{~s}, 3 \mathrm{H}, \mathrm{CH}_{3}\right) .{ }^{13} \mathrm{C}$ NMR (100 MHz, $\left.\mathrm{CDCl}_{3}\right): \delta 127.2(\mathrm{C}), 124.8(\mathrm{CH}), 124.6(\mathrm{C}), 123.3(\mathrm{C}), 66.1\left(\mathrm{CH}_{2}\right)$, $65.5\left(\mathrm{CH}_{2}\right), 19.7\left(\mathrm{CH}_{3}\right), 19.4\left(\mathrm{CH}_{3}\right)$.

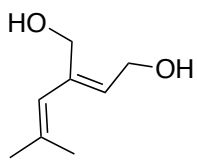

${ }^{1} \mathbf{H}$ NMR $\left(400 \mathrm{MHz}, \mathrm{CDCl}_{3}\right): \delta 5.78(\mathrm{~s}, 1 \mathrm{H}, \mathrm{CH}), 5.62(\mathrm{~s}, 1 \mathrm{H}, \mathrm{CH}), 4.75(\mathrm{br} \mathrm{s}, 2 \mathrm{H}$, $\mathrm{CH}_{2}$ ), 4.67 (br s, $2 \mathrm{H}, \mathrm{CH}_{2}$ ), 1.85 (s, 3H, $\left.\mathrm{CH}_{3}\right), 1.76$ (s, 3H, $\left.\mathrm{CH}_{3}\right) .{ }^{13} \mathbf{C}$ NMR (100 MHz, $\left.\mathrm{CDCl}_{3}\right): \delta 137.7(\mathrm{C}), 136.9(\mathrm{C}), 122.5(\mathrm{CH}), 117.7(\mathrm{CH}), 76.5\left(\mathrm{CH}_{2}\right), 75.4\left(\mathrm{CH}_{2}\right), 27.2$ $\left(\mathrm{CH}_{3}\right), 20.1\left(\mathrm{CH}_{3}\right)$. MS (EI) $\mathrm{m} / z$ calculated for $\mathrm{C}_{8} \mathrm{H}_{14} \mathrm{O}_{2}: 142.0994[\mathrm{M}]^{+}$; found: 142.0835 . 


\section{Cycloisomerization of enyne 21}

Following the general procedure, gold carbene 5ab (4 mg, $0.005 \mathrm{mmol}, 0.03$ equiv) and NaBArF (5 mg, 0.005 mmol, 0.03 equiv) were dissolved in $1 \mathrm{~mL}$ anhydrous $\mathrm{CH}_{2} \mathrm{Cl}_{2}$ under argon for $15 \mathrm{~min}$. Enyne 21 (43 mg, $0.17 \mathrm{mmol}, 1.00$ equiv) was added dissolved in $1 \mathrm{~mL}$ of anhydrous $\mathrm{CH}_{2} \mathrm{Cl}_{2}$. After $3 \mathrm{~h}$ of stirring at $\mathrm{rt}$, the reaction was filtered through a short pad of celite and $\mathrm{SiO}_{2}$. The crude was purified through a short pad of $\mathrm{SiO}_{2}$ (hexane:AcOEt 98:2) to obtain 22 as white solid (21 mg, 50\%).

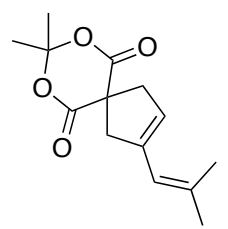

${ }^{1} \mathbf{H}$ NMR $\left(400 \mathrm{MHz}, \mathrm{CDCl}_{3}\right): \delta 5.73,(\mathrm{~s}, 1 \mathrm{H}, \mathrm{CH}), 5.43(\mathrm{~s}, 1 \mathrm{H}, \mathrm{CH}), 3.27\left(\mathrm{~s}, 2 \mathrm{H}, \mathrm{CH}_{2}\right)$, $3.16\left(\mathrm{~s}, 2 \mathrm{H}, \mathrm{CH}_{2}\right), 1.82\left(\mathrm{~s}, 3 \mathrm{H}, \mathrm{CH}_{3}\right), 1.80\left(\mathrm{~s}, 3 \mathrm{H}, \mathrm{CH}_{3}\right), 1.76\left(\mathrm{~s}, 6 \mathrm{H}, 2 \mathrm{CH}_{3}\right) .{ }^{13} \mathbf{C} \mathbf{N M R}$ $\left(100 \mathrm{MHz}, \mathrm{CDCl}_{3}\right): \delta 171.0(2 \mathrm{C}=\mathrm{O}), 138.7(\mathrm{C}), 137.2(\mathrm{C}), 123.0(\mathrm{C}), 119.6(\mathrm{CH}), 105.0$ (C), $51.6(\mathrm{C}), 48.6\left(\mathrm{CH}_{2}\right), 46.5\left(\mathrm{CH}_{2}\right), 29.1\left(\mathrm{CH}_{3}\right), 29.0\left(\mathrm{CH}_{3}\right), 27.4\left(\mathrm{CH}_{3}\right), 20.1\left(\mathrm{CH}_{3}\right)$. IR (KBr): $\boldsymbol{v}_{\text {máx }} 3283,1765,1738,1345,1269,1102,959,692$. MS (ESI) $\mathrm{m} / z$ calculated for $\mathrm{C}_{14} \mathrm{H}_{18} \mathrm{NaO}_{4}: 273.1097$ [M+Na] $]^{+}$; found: 273.1095. mp: melts below $30{ }^{\circ} \mathrm{C}$.

\section{Crystal data:}

All data were collected at low temperature using oil-coated shock-cooled crystals on a Bruker-AXS APEX II diffractometer with MoKa radiation $(\lambda=0.71073 \AA)$. The structures were solved by direct methods ${ }^{11}$ and all non hydrogen atoms were refined anisotropically using the least-squares method on $\mathrm{F}^{2} .{ }^{12}$ The absolute structure parameters have been refined using the Flack-method. ${ }^{13}$ CCDC 1463903 (5aa), 1463904 (5ab), 1463905 (5ac) and 1463906 (5ca) contain the supplementary crystallographic data for this paper. These data are provided free of charge by The Cambridge Crystallographic Data Centre.

\footnotetext{
${ }^{11}$ Sheldrick, G. M.; Acta Crystallogr. A46, 1990 467-473.

${ }^{12}$ Sheldrick, G. M. Acta Crystallogr. A64, 2008, 112-122.

${ }^{13}$ (a) Flack, H. D. Acta Crystallogr. A39, 1983, 876; (b) Parsons, S.; Flack, H. D.; Wagner, T. Acta Cryst. B69, 2013, 249.
} 
Crystal data for 5aa:

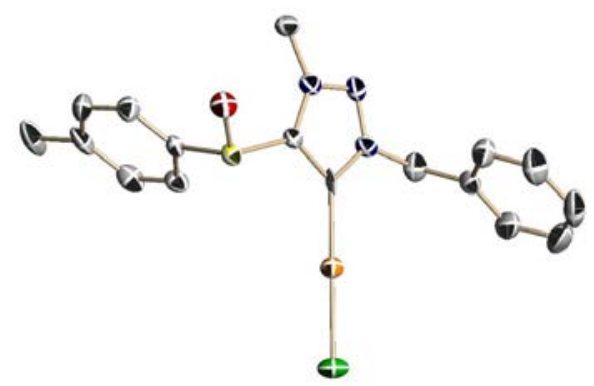

$\mathrm{C}_{17} \mathrm{H}_{17} \mathrm{AuClN}_{3} \mathrm{OS}, \mathrm{Mr}=543.81$, crystal dimensions $0.5 \times 0.3 \times 0.3 \mathrm{~mm}^{3}$, triclinic, $P \overline{\mathrm{I}}, a$ $=5.087(1) \AA, b=7.868(1) \AA, c=12.413(1) \AA, \alpha=81.440(3)^{\circ}, \beta=85.701(4)^{\circ}, \gamma=$ $73.075(3)^{\circ}$, cell volume $=469.72(6) \AA^{3}, Z=1, \varrho_{\text {calcd }}=1.922 \mathrm{Mg} / \mathrm{m}^{3}, \mu=8.092 \mathrm{~mm}^{-1}, \mathrm{~T}$ $=173(2) \mathrm{K}, 2 \theta_{\max }=56.5^{\circ}, 22411$ reflections collected, 3885 independent, $\mathrm{R}_{\mathrm{int}}=0.0466$, $\mathrm{R} 1=0.0258$ and $\mathrm{wR} 2=0.0548$ for $\mathrm{I}>2 \sigma(\mathrm{I}), \mathrm{R} 1=0.0261$ and $\mathrm{wR} 2=0.0550$ for all data, residual electron density $=1.877 \mathrm{eA}^{-3}$, absolute structure parameter $\mathrm{x}=-0.013(7)$.

Crystal data for $\mathbf{5 a b}$

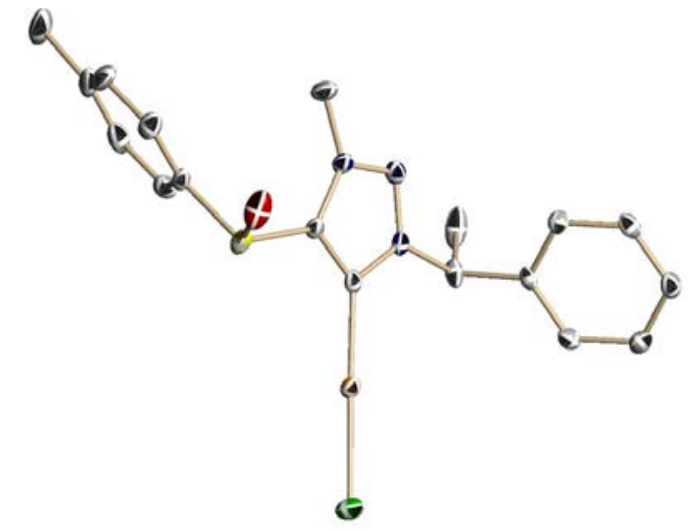

$\mathrm{C}_{18} \mathrm{H}_{19} \mathrm{AuClN}{ }_{3} \mathrm{OS}, \mathrm{Mr}=557.84$, crystal dimensions $0.4 \times 0.3 \times 0.3 \mathrm{~mm}^{3}$, orthorhombic, $P 2{ }_{1}{ }_{1}{ }_{1}, a=7.5537(2) \AA, b=14.1240(5) \AA, c=18.0020(6) \AA$, cell volume $=1920.6(2)$ $\AA^{3}, \mathrm{Z}=4, \varrho_{\text {calcd }}=1.929 \mathrm{Mg} / \mathrm{m}^{3}, \mu=7.918 \mathrm{~mm}^{-1}, \mathrm{~T}=100(2) \mathrm{K}, 2 \theta_{\max }=56.5^{\circ}, 19840$ reflections collected, 4737 independent, $\mathrm{R}_{\mathrm{int}}=0.0208, \mathrm{R} 1=0.0104$ and $\mathrm{wR} 2=0.0240$ for $\mathrm{I}>2 \sigma(\mathrm{I}), \mathrm{R} 1=0.0108$ and $\mathrm{wR} 2=0.0241$ for all data, residual electron density $=$ $0.302 \mathrm{eA}^{-3}$, absolute structure parameter $\mathrm{x}=-0.019(2)$. 
Crystal data for 5ac:

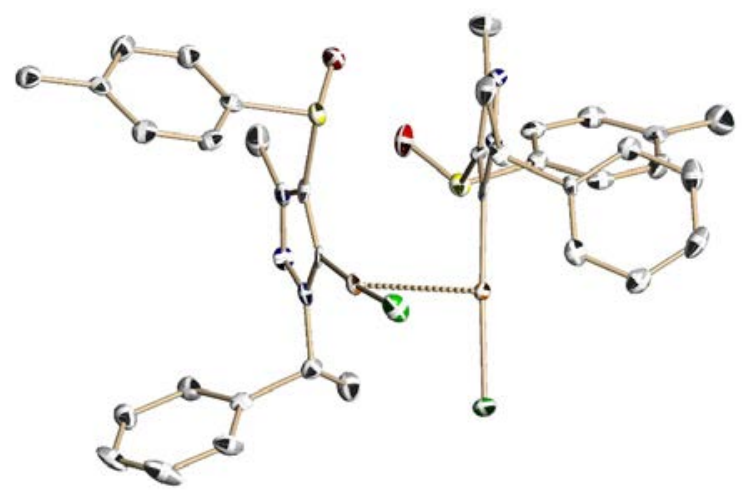

$\mathrm{C}_{18} \mathrm{H}_{19} \mathrm{AuClN}{ }_{3} \mathrm{OS}, \mathrm{Mr}=557.84$, crystal dimensions $0.4 \times 0.2 \times 0.2 \mathrm{~mm}^{3}$, orthorhombic, $P 2{ }_{1}{ }_{1}{ }_{1}, a=10.9737(4) \AA, b=17.4658(7) \AA, c=19.9371(7) \AA$, cell volume = $3821.2(2) \AA^{3}, \mathrm{Z}=8, \varrho_{\text {calcd }}=1.939 \mathrm{Mg} / \mathrm{m}^{3}, \mu=7.960 \mathrm{~mm}^{-1}, \mathrm{~T}=100(2) \mathrm{K}, 2 \theta_{\max }=55.5^{\circ}$, 39141 reflections collected, 7919 independent, $\mathrm{R}_{\text {int }}=0.0795, \mathrm{R} 1=0.0498$ and $\mathrm{wR} 2=$ 0.0607 for $\mathrm{I}>2 \sigma(\mathrm{I}), \mathrm{R} 1=0.0649$ and $\mathrm{wR} 2=0.0639$ for all data, residual electron density $=3.896 \mathrm{eA}^{-3}$, absolute structure parameter $\mathrm{x}=-0.019(6)$.

Crystal data for 5ca:

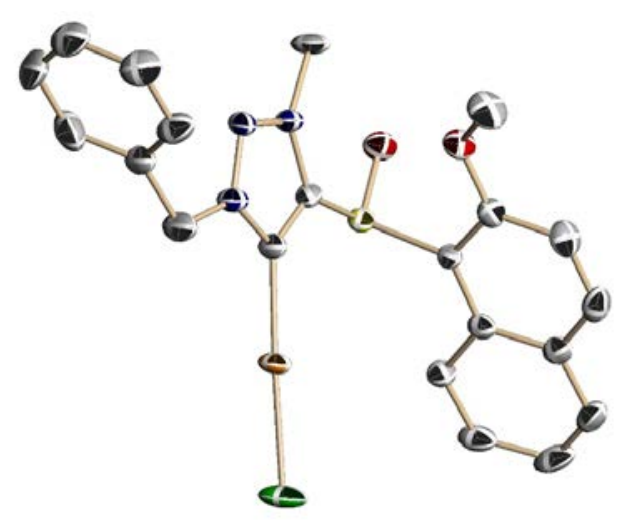

$\mathrm{C}_{21} \mathrm{H}_{19} \mathrm{AuClN}_{3} \mathrm{O}_{2} \mathrm{~S}, \mathrm{Mr}=609.87$, crystal dimensions $0.5 \times 0.1 \times 0.05 \mathrm{~mm}^{3}$,

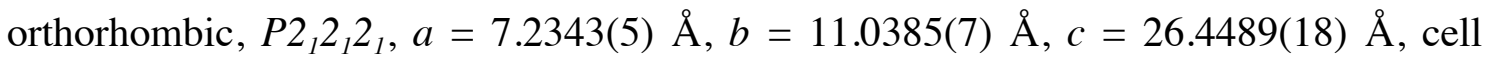
volume $=2112.1(2) \AA^{3}, \mathrm{Z}=4, \varrho_{\text {calcd }}=1.918 \mathrm{Mg} / \mathrm{m}^{3}, \mu=7.213 \mathrm{~mm}^{-1}, \mathrm{~T}=173(2) \mathrm{K}$, $2 \theta_{\max }=56.5^{\circ}, 42989$ reflections collected, 5159 independent, $\mathrm{R}_{\mathrm{int}}=0.0788, \mathrm{R} 1=0.0305$ and $w R 2=0.0512$ for $\mathrm{I}>2 \sigma(\mathrm{I}), \mathrm{R} 1=0.0470$ and $\mathrm{wR} 2=0.0549$ for all data, residual electron density $=1.449 \mathrm{eA}^{-3}$, absolute structure parameter $\mathrm{x}=-0.011(6)$. 

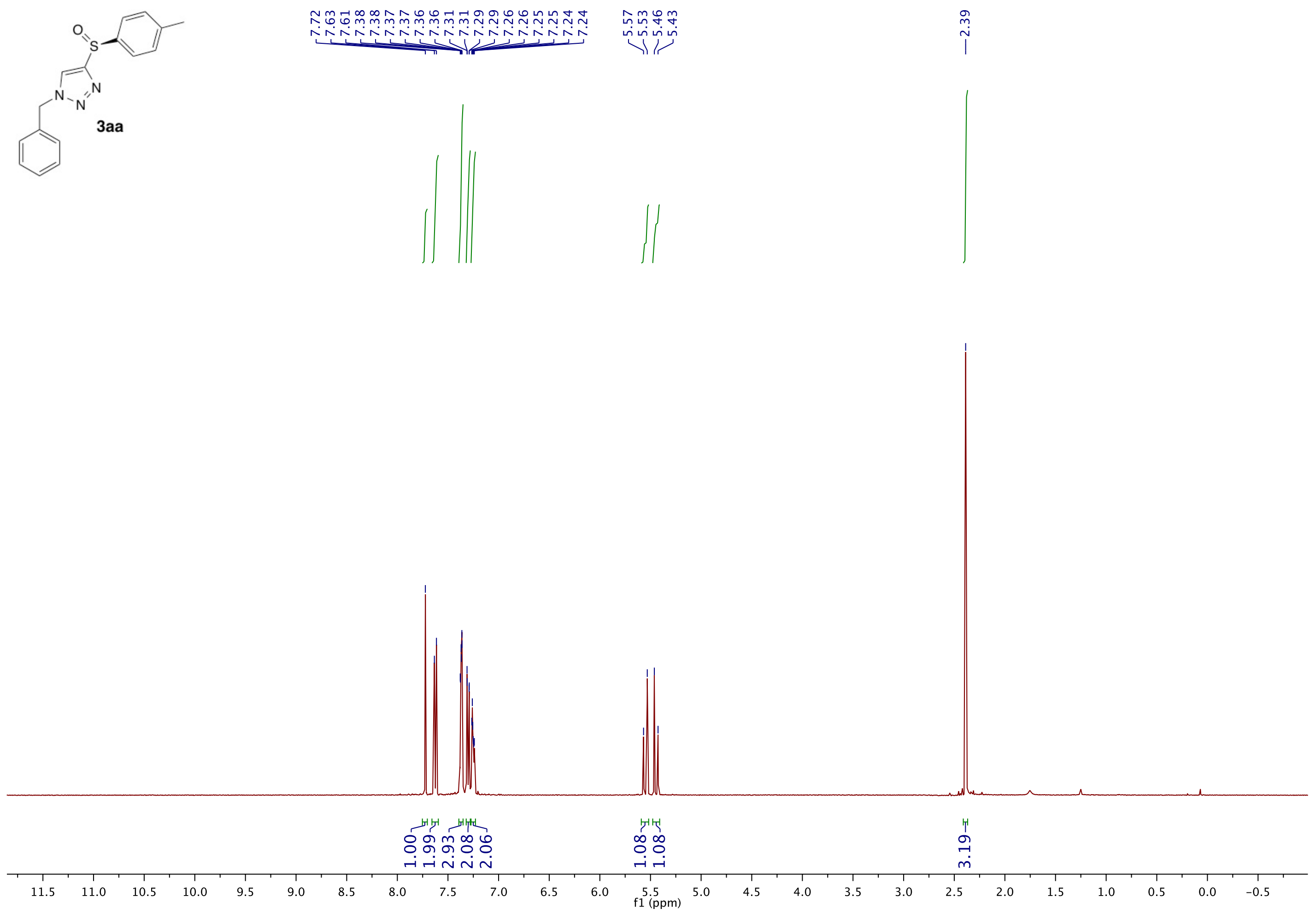

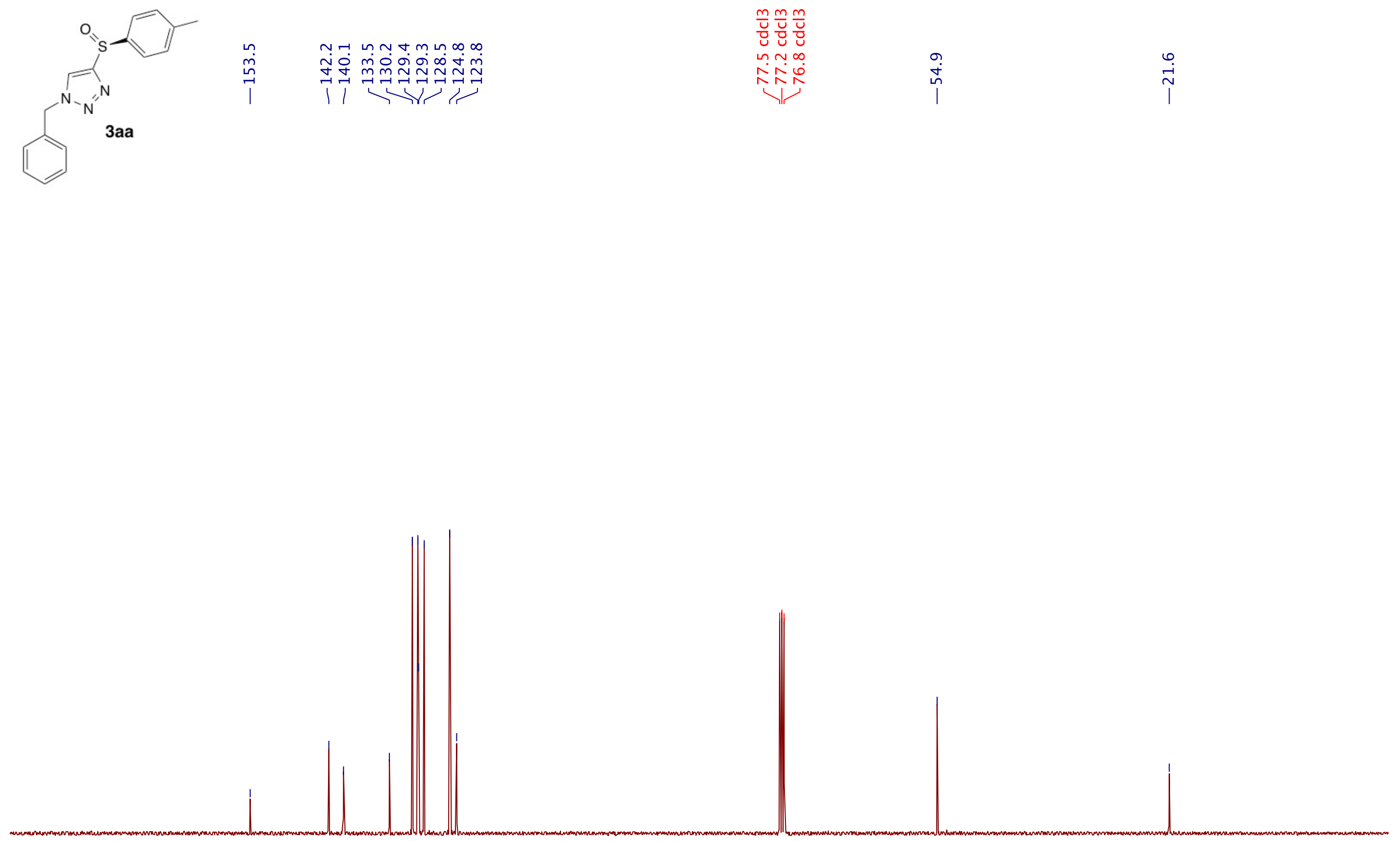

\begin{tabular}{|c|c|c|c|c|c|c|c|c|c|c|c|c|c|c|c|c|c|c|}
\hline & 1 & & 1 & 1 & 1 & 1 & 1 & 1 & 1 & 1 & 1 & 1 & 1 & 1 & 1 & 1 & 1 & $T$ \\
\hline 180 & 170 & 160 & 150 & 140 & 130 & 120 & 110 & 100 & $\begin{array}{c}90 \\
\mathrm{f} 1(\mathrm{ppm})\end{array}$ & 80 & 70 & 60 & 50 & 40 & 30 & 20 & 10 & 0 \\
\hline
\end{tabular}




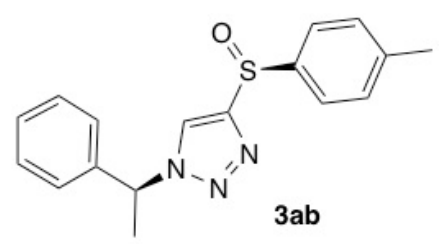

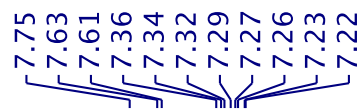

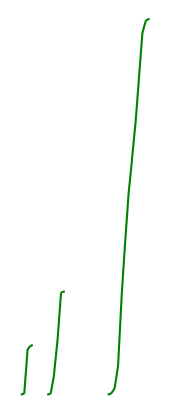

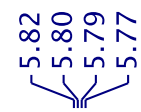

f
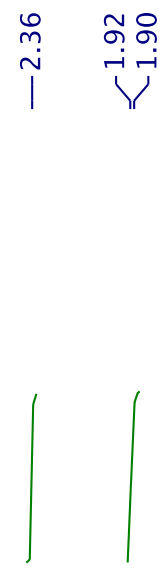

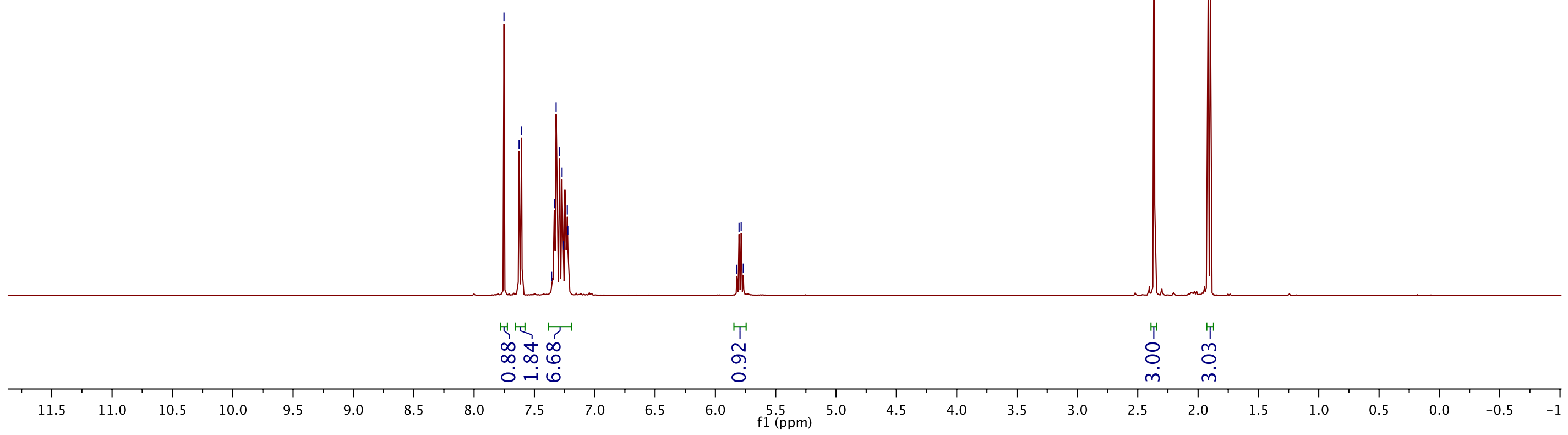




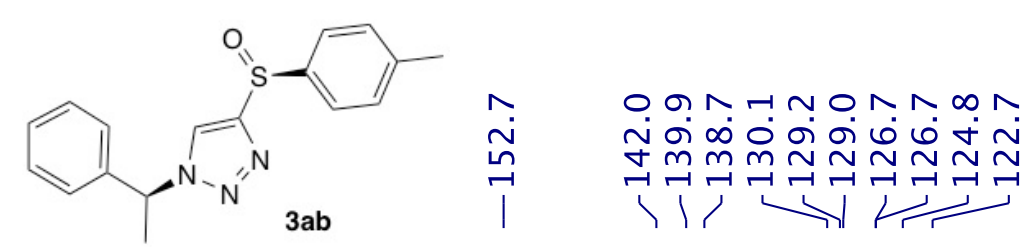

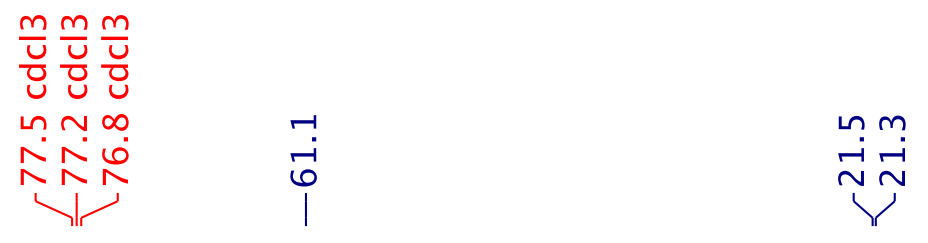

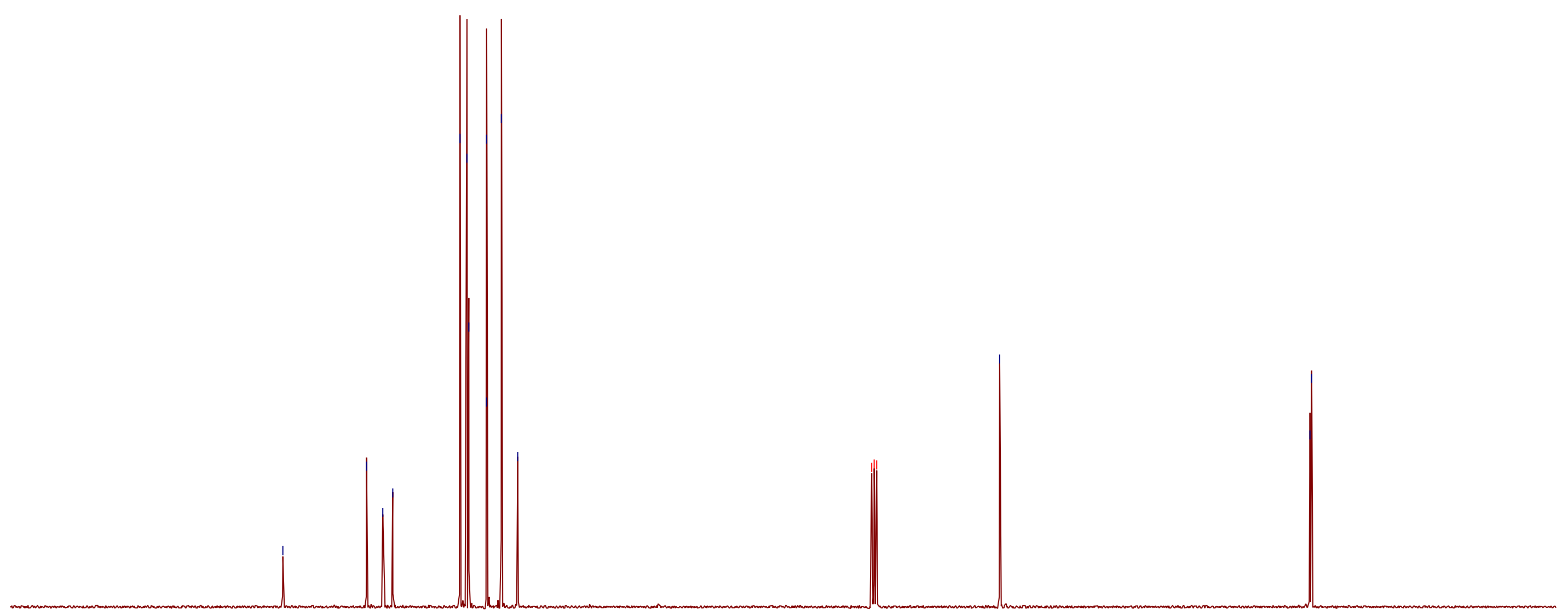

\begin{tabular}{|c|c|c|c|c|c|c|c|c|c|c|c|c|c|c|c|c|c|c|}
\hline 1 & 1 & 1 & 1 & 1 & 1 & 1 & 1 & 1 & 1 & 1 & 1 & 1 & 1 & 1 & 1 & 1 & 1 & $T$ \\
\hline 180 & 170 & 160 & 150 & 140 & 130 & 120 & 110 & 100 & $\begin{array}{c}90 \\
\mathrm{f} 1(\mathrm{ppm})\end{array}$ & 80 & 70 & 60 & 50 & 40 & 30 & 20 & 10 & 0 \\
\hline
\end{tabular}



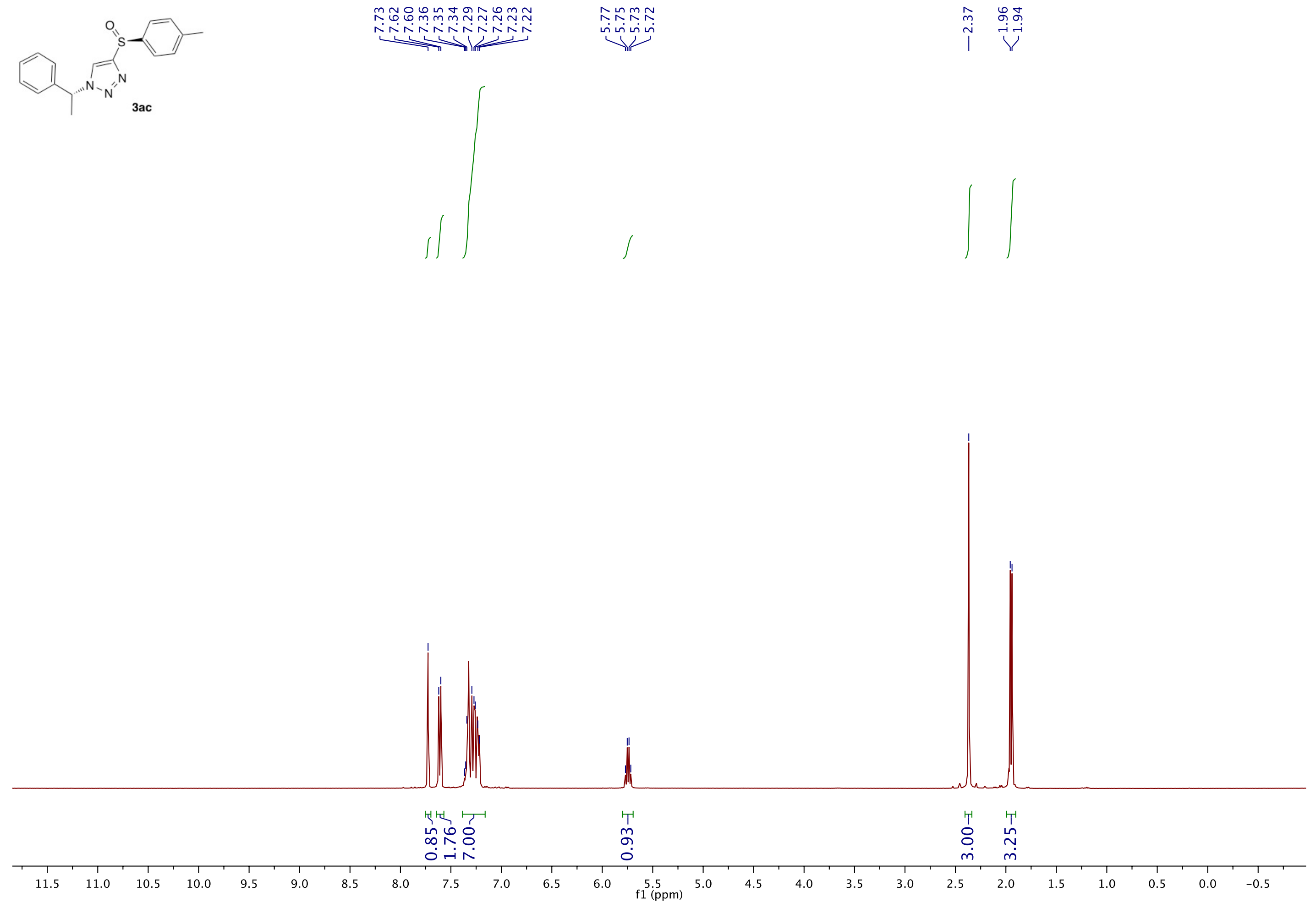

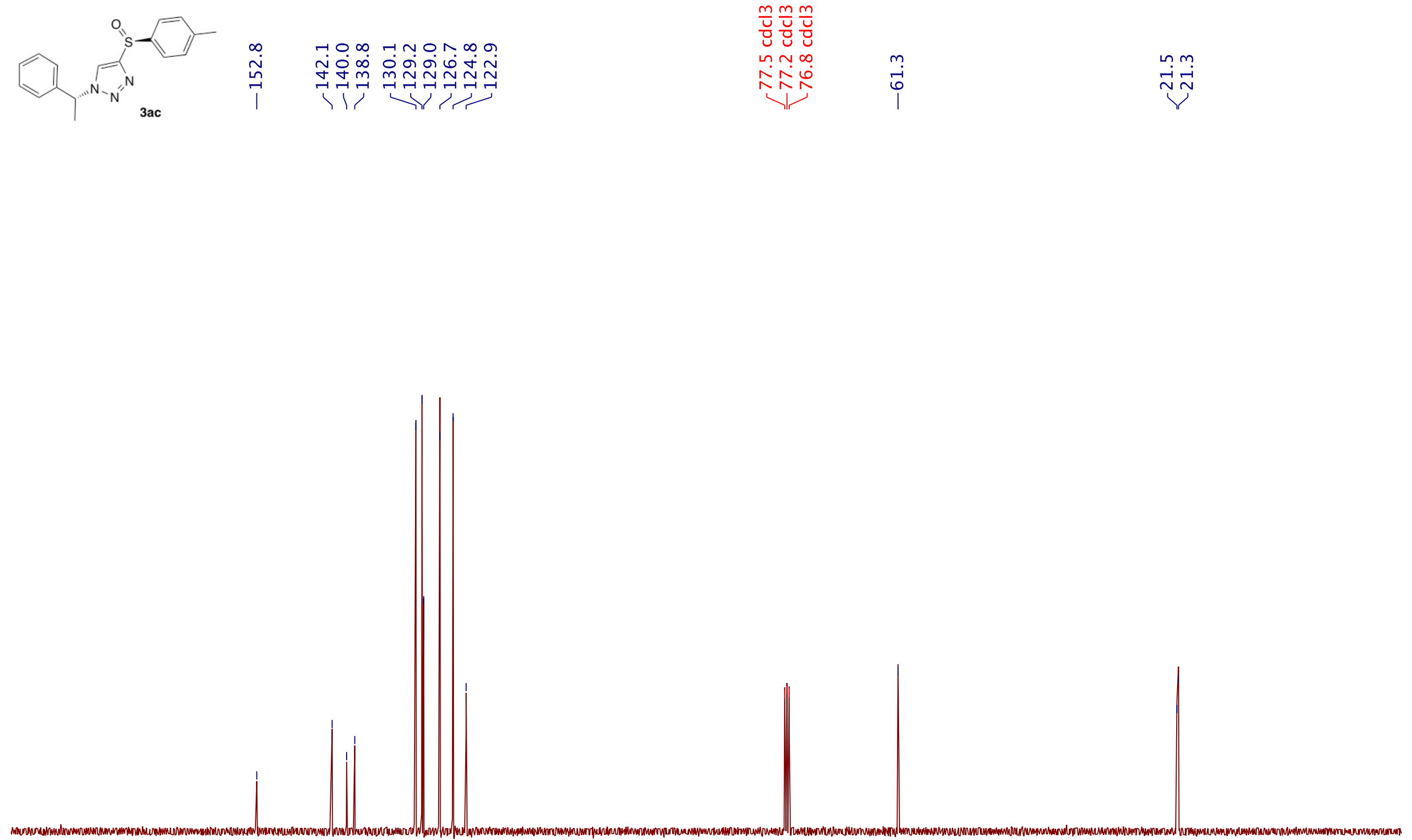

\begin{tabular}{|c|c|c|c|c|c|c|c|c|c|c|c|c|c|c|c|c|c|c|c|}
\hline 1 & 17 & 1 & 1 & 1 & 11 & 11 & 11 & 1 & 1 & 1 & 7 & 1 & 1 & 1 & 11 & 12 & 10 & 1 & -1 \\
\hline 180 & 170 & 160 & 150 & 140 & 130 & 120 & 110 & 100 & $\begin{array}{l}90 \\
\mathrm{f} 1(\mathrm{ppm})\end{array}$ & 80 & 70 & 60 & 50 & 40 & 30 & 20 & 10 & 0 & -1 \\
\hline
\end{tabular}




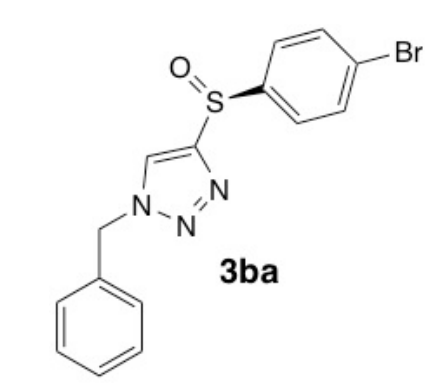

$\int_{11}^{1}$

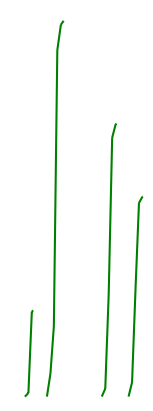

$\left.\int\right\}$

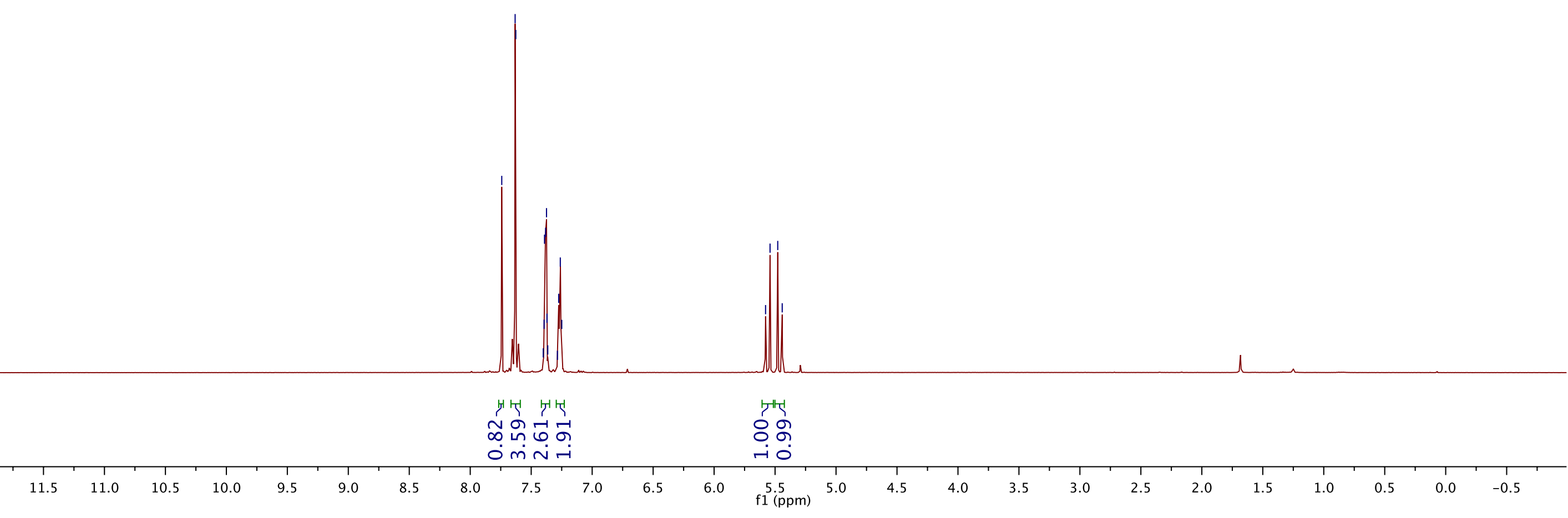




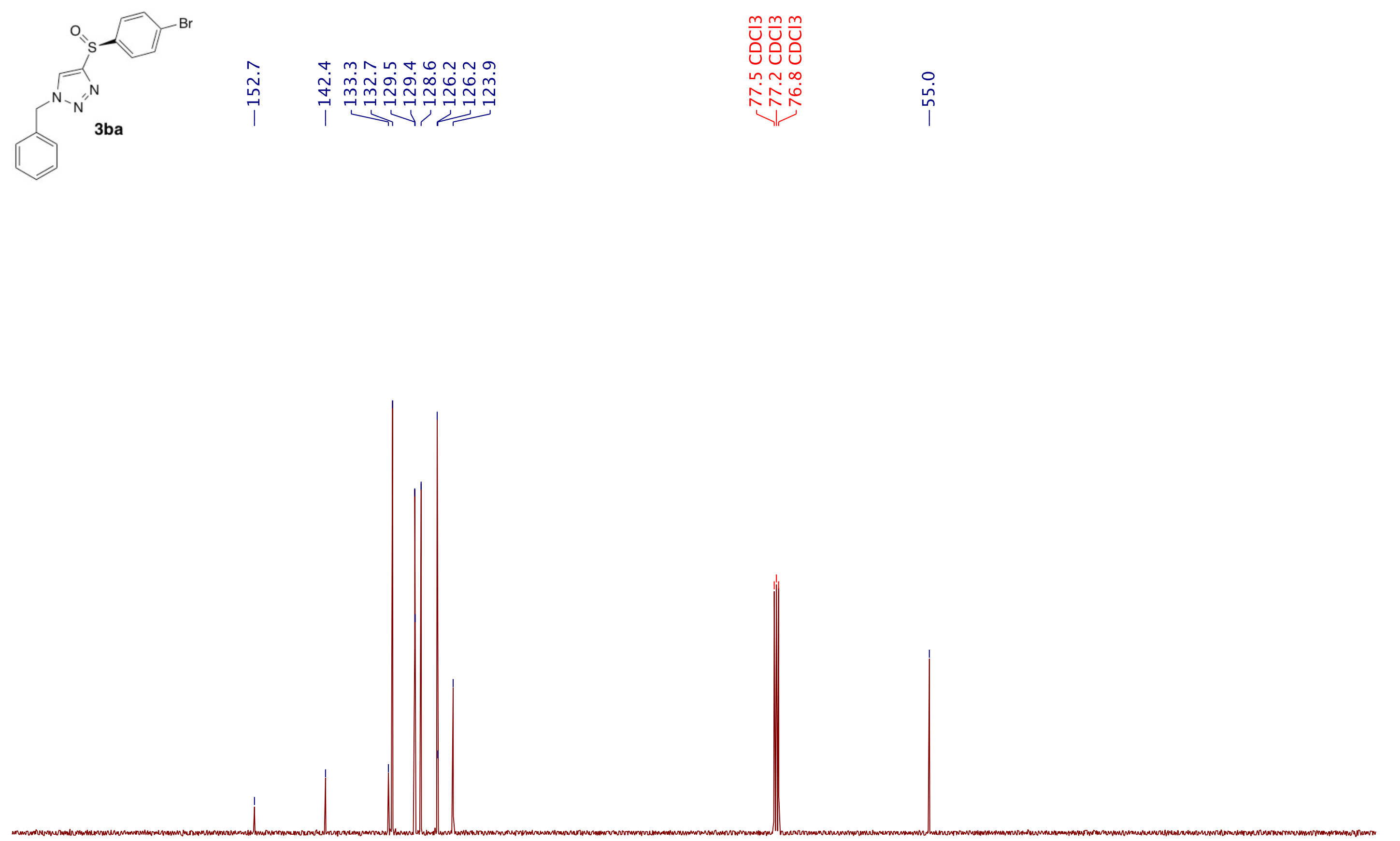



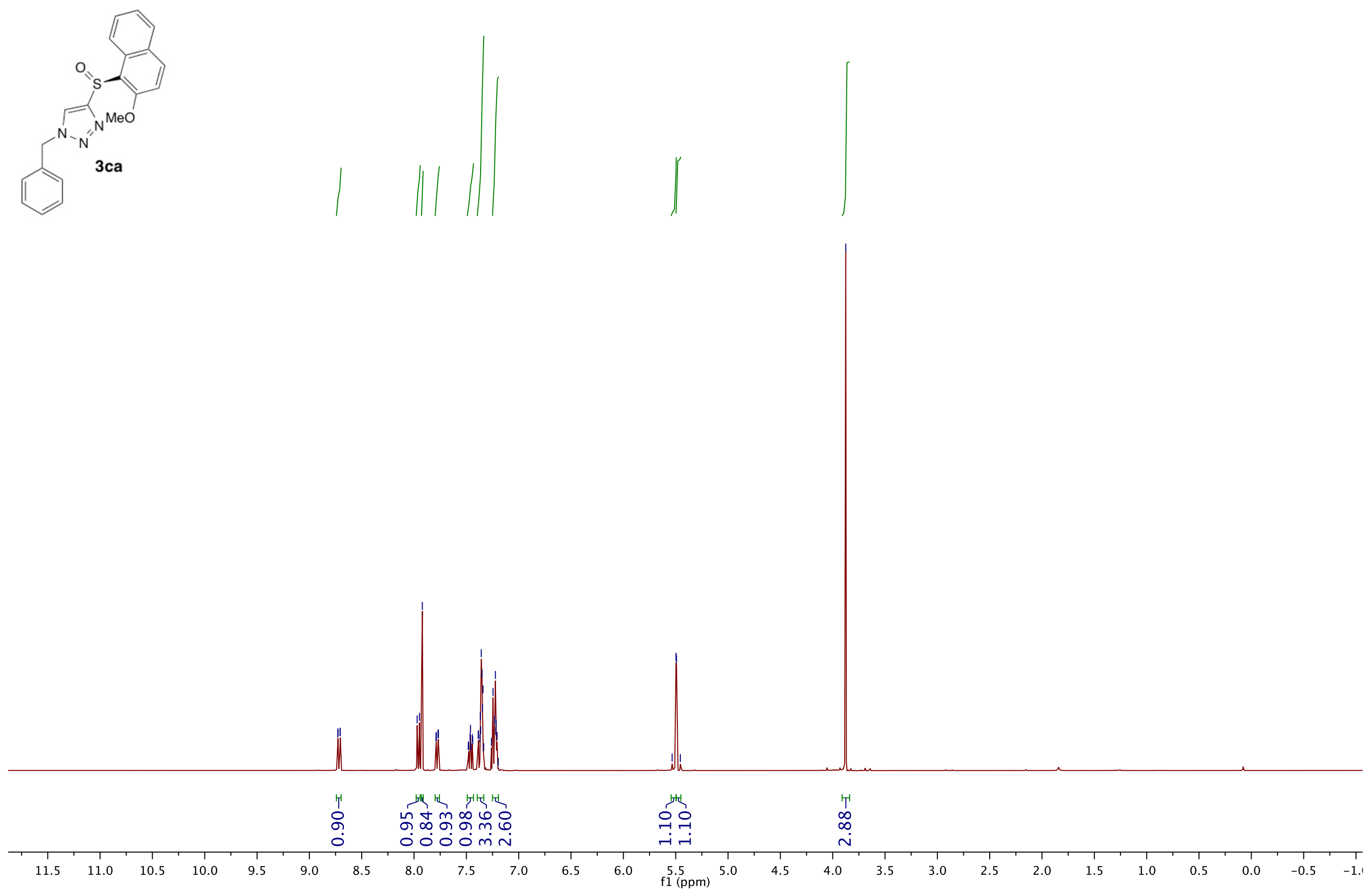


$$
\begin{aligned}
& \text { 6) ๆ } \\
& \text { 슴 듬 }
\end{aligned}
$$

3ca

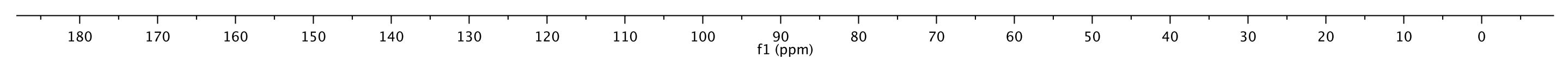




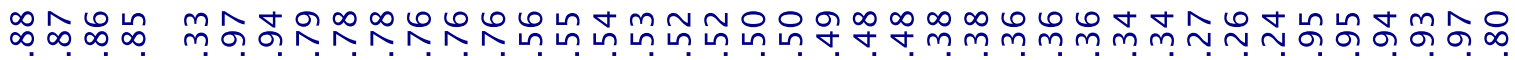

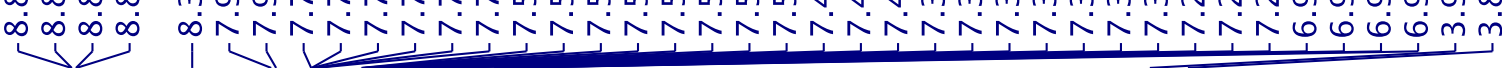
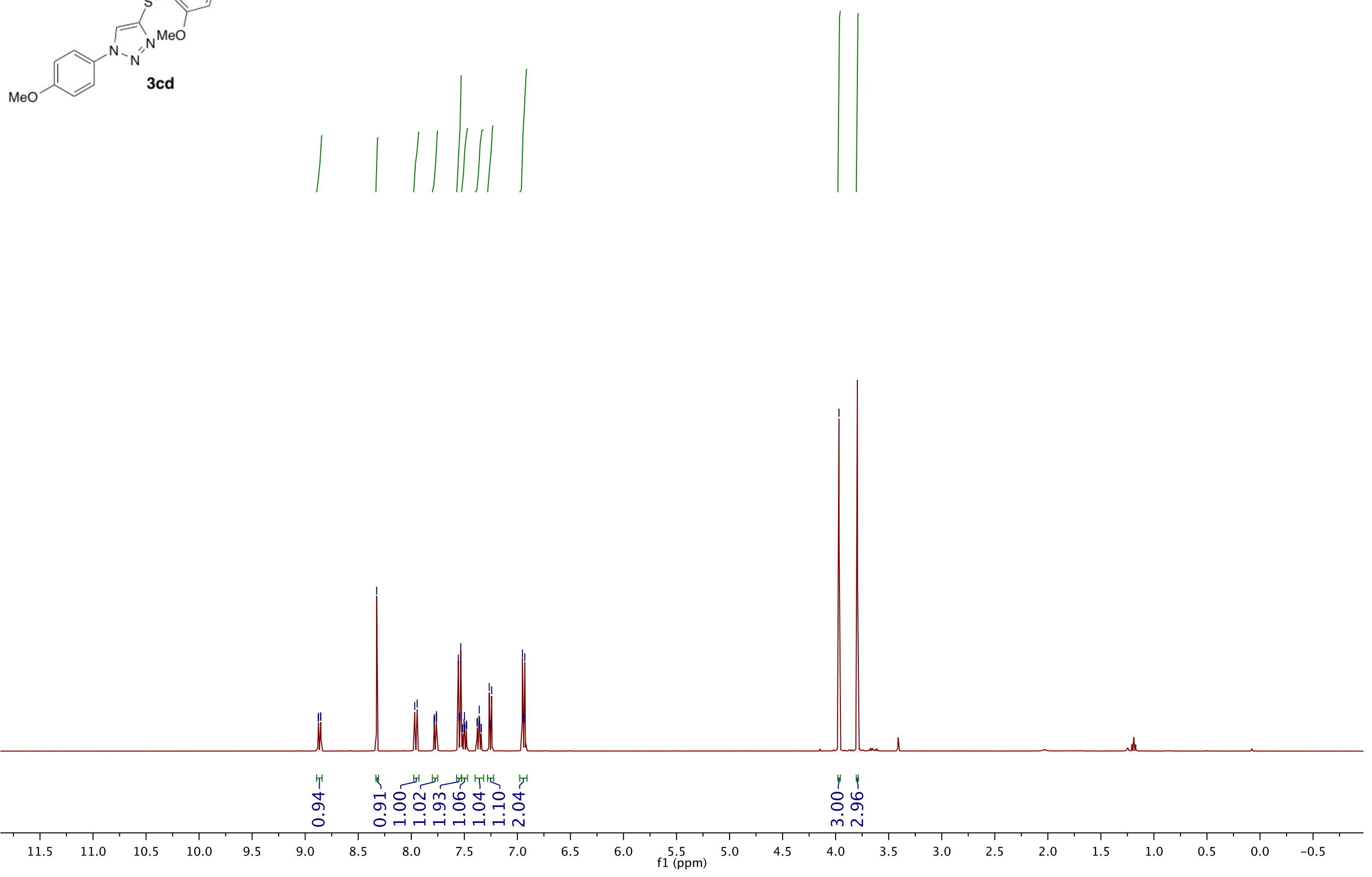


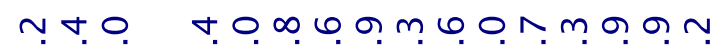

ชิ่น

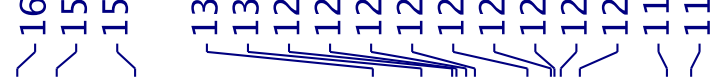
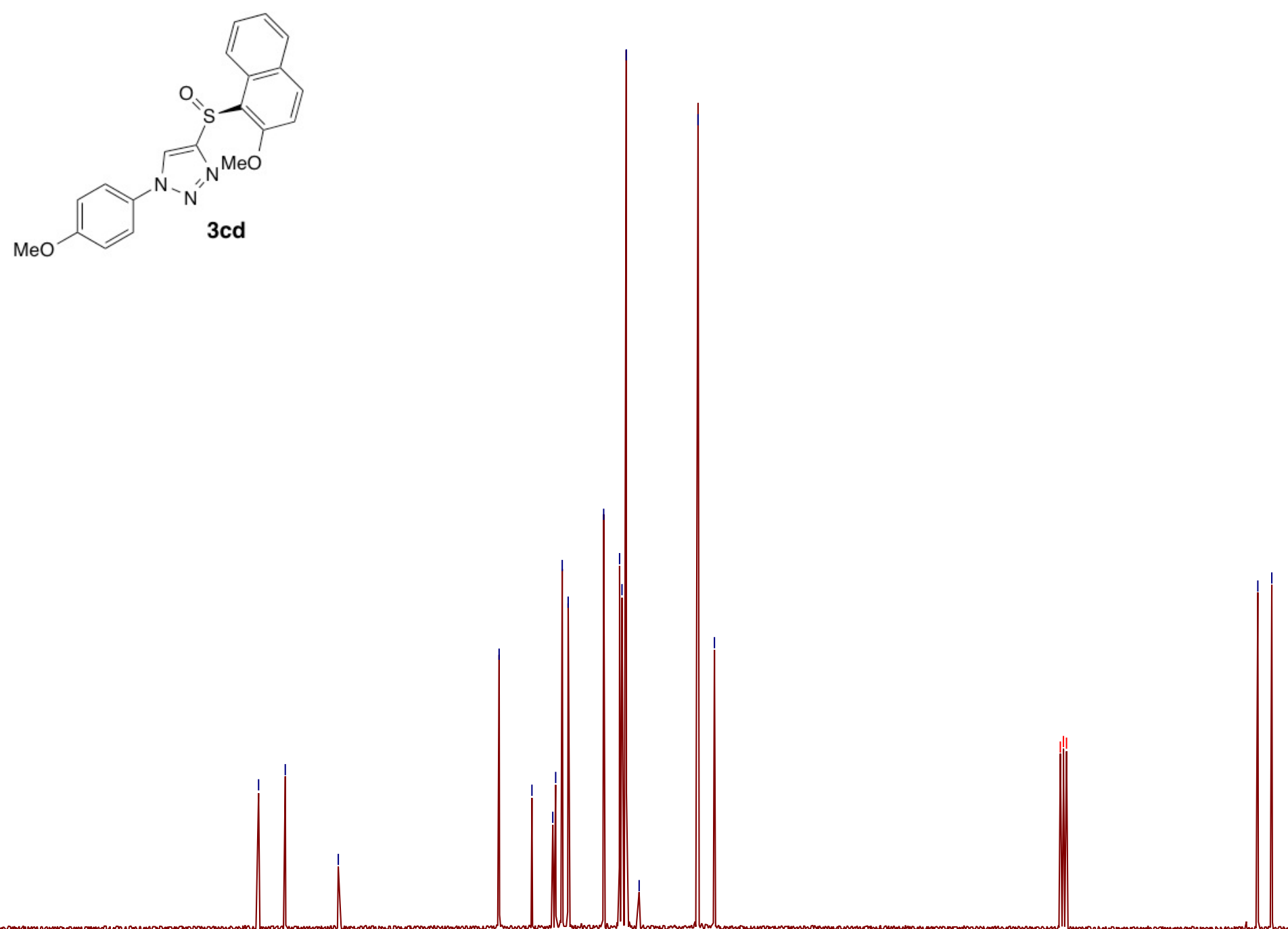

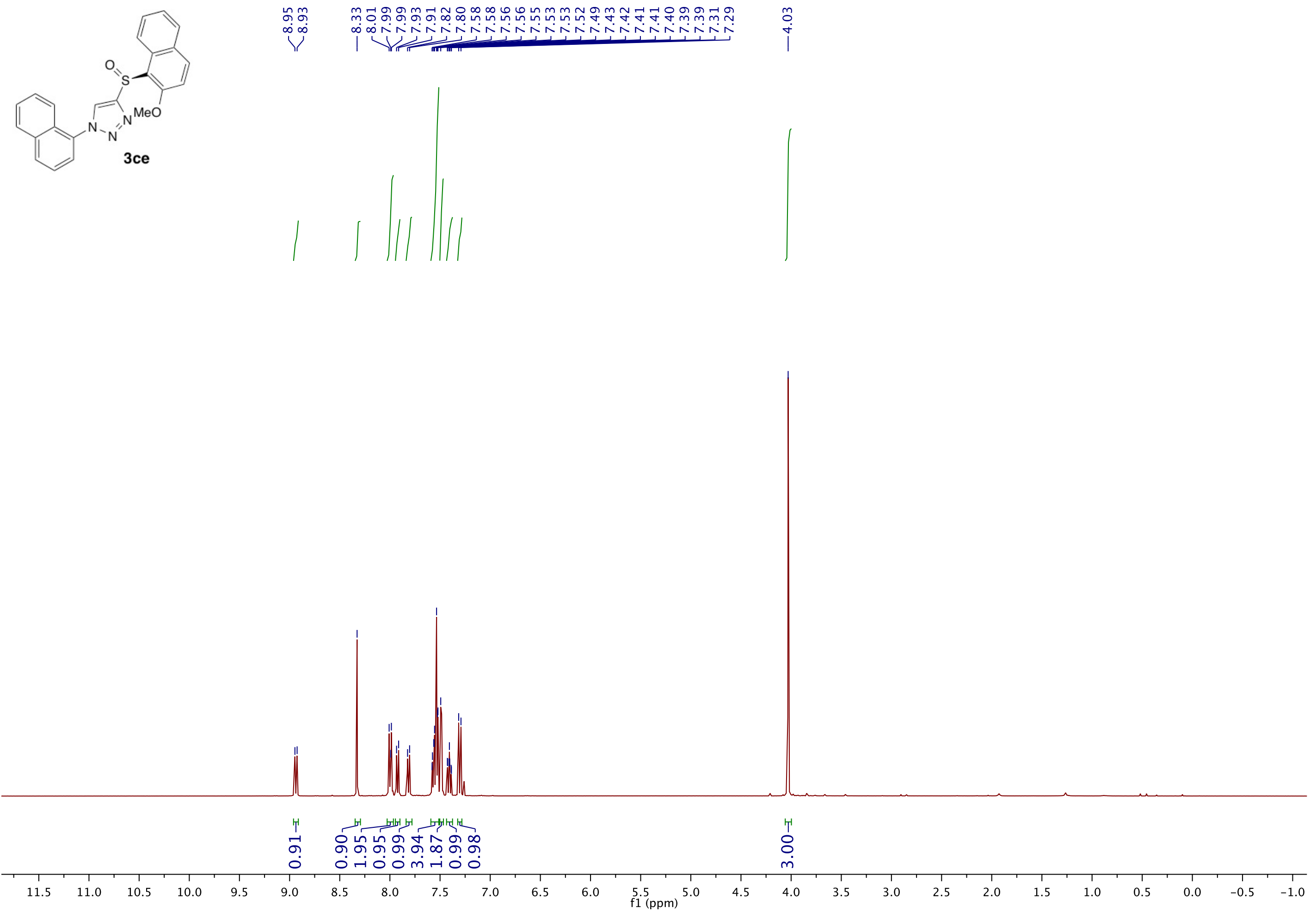

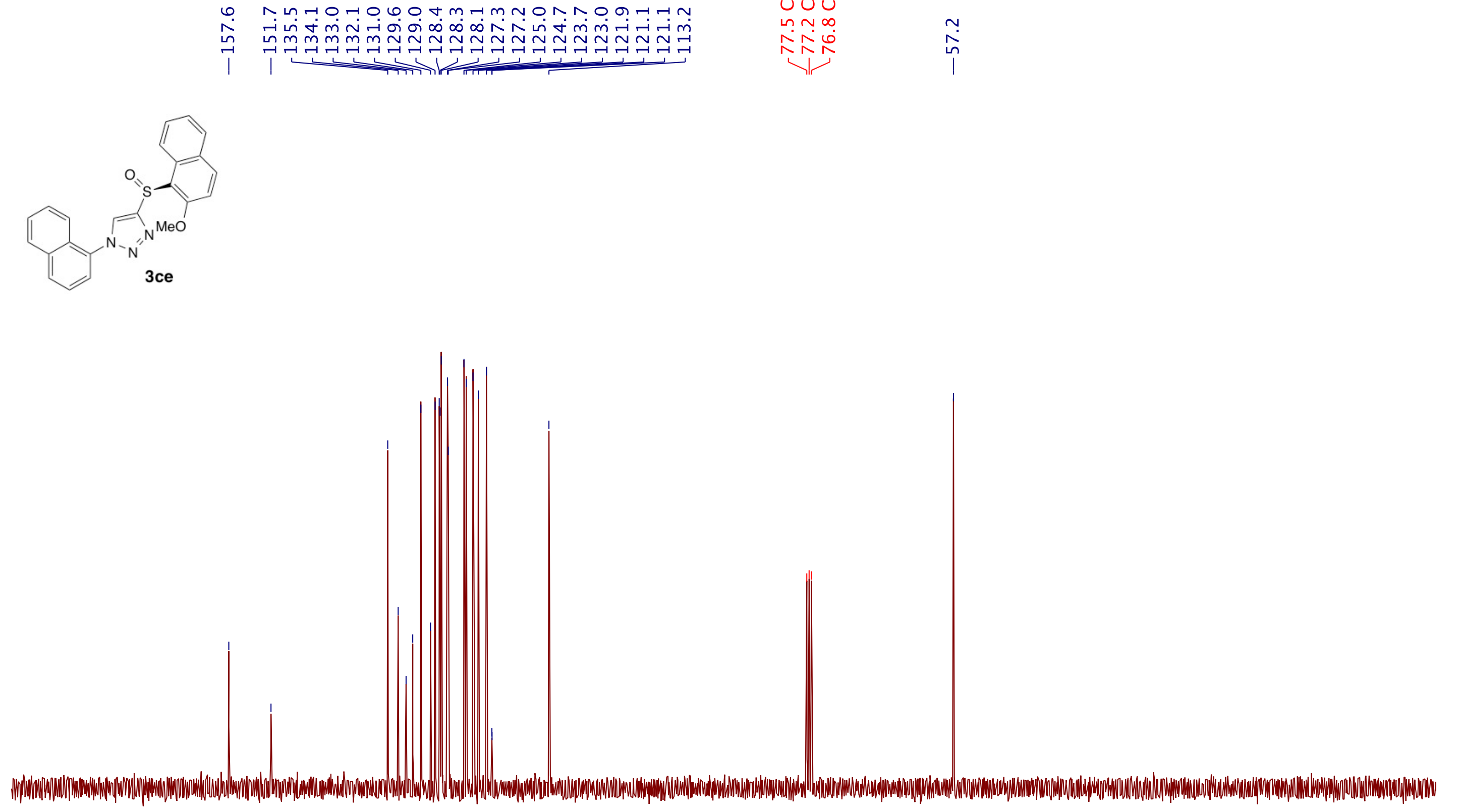

\begin{tabular}{|c|c|c|c|c|c|c|c|c|c|c|c|c|c|c|c|c|c|c|}
\hline 1 & 1 & 1 & 1 & 1 & 1 & 1 & 1 & 1 & 1 & 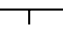 & $T$ & 1 & $T$ & 1 & 1 & 1 & 1 & $T$ \\
\hline 180 & 170 & 160 & 150 & 140 & 130 & 120 & 110 & 100 & $\begin{array}{c}90 \\
\mathrm{f1}(\mathrm{ppm})\end{array}$ & 80 & 70 & 60 & 50 & 40 & 30 & 20 & 10 & 0 \\
\hline
\end{tabular}



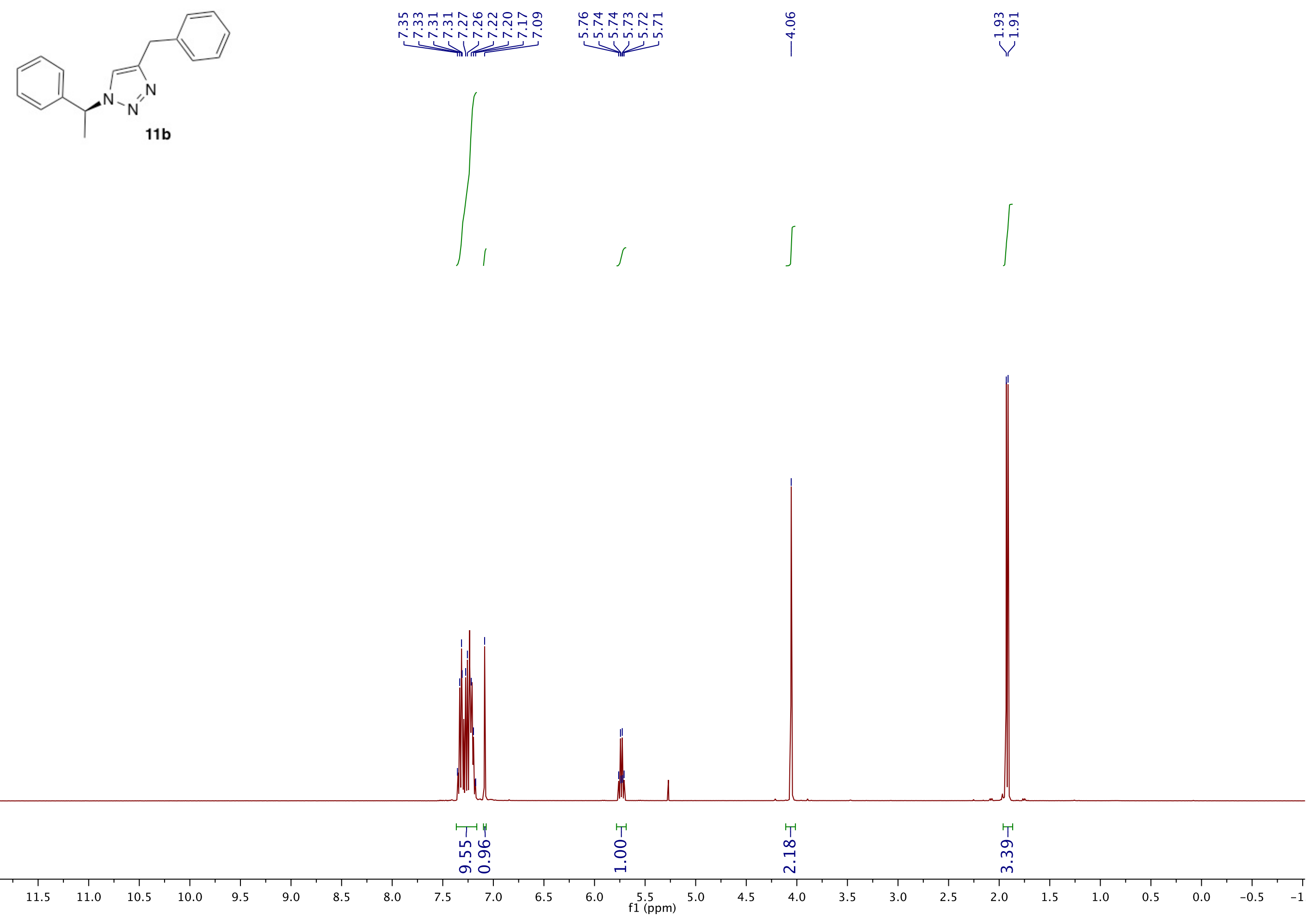

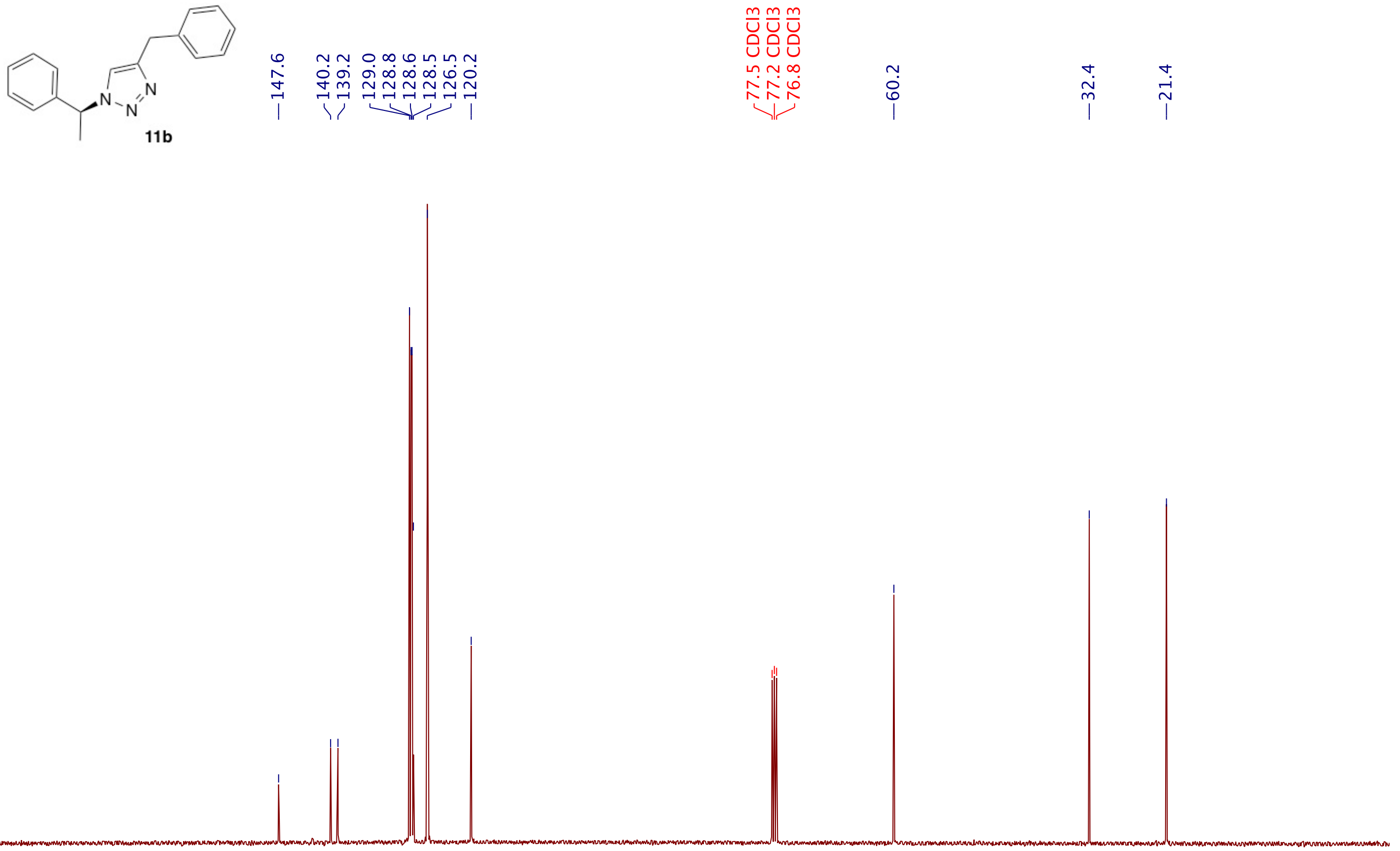

\begin{tabular}{|c|c|c|c|c|c|c|c|c|c|c|c|c|c|c|c|c|c|c|}
\hline 180 & 170 & 160 & 150 & 140 & 130 & 120 & 110 & 100 & $\begin{array}{c}90 \\
\text { f1 (ppm) }\end{array}$ & 80 & 70 & 60 & 50 & 40 & 30 & 20 & 10 & -1 \\
\hline
\end{tabular}




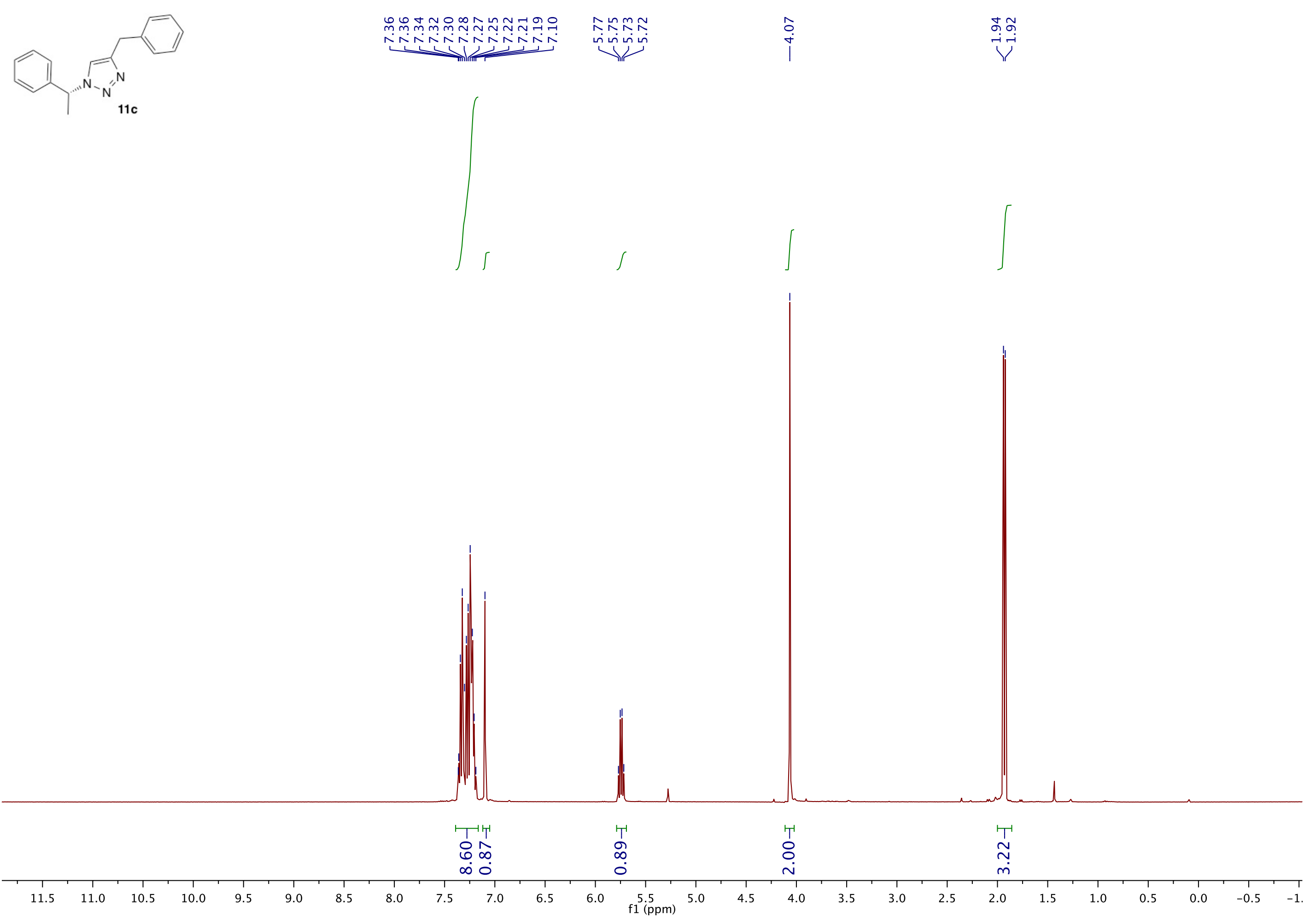



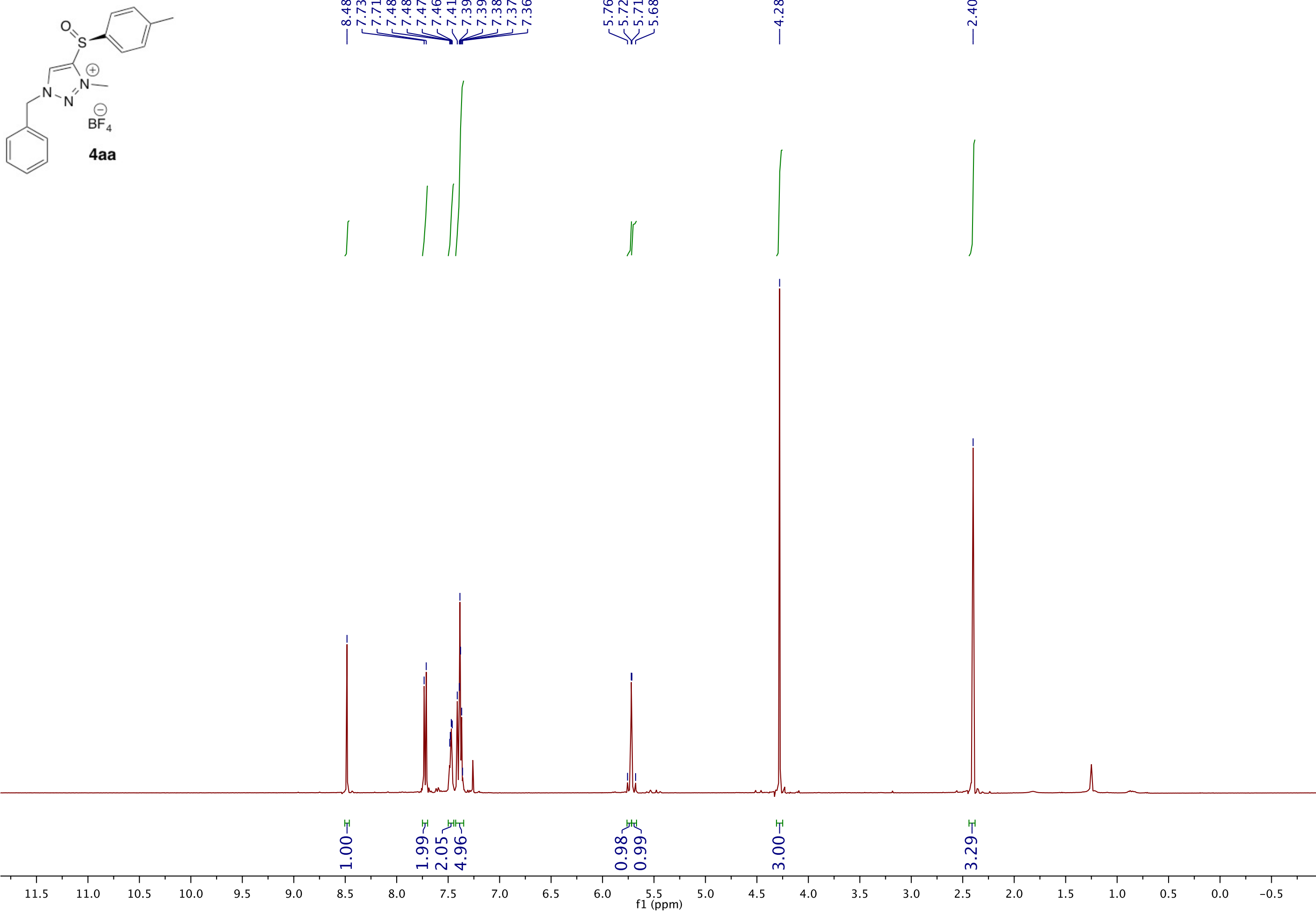
<smiles></smiles>

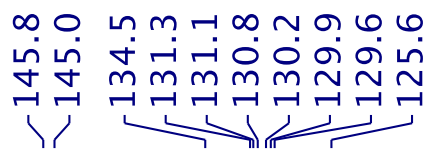

$\stackrel{+}{\infty}$

$\stackrel{0}{m}$

$\stackrel{\sim}{\sim}$
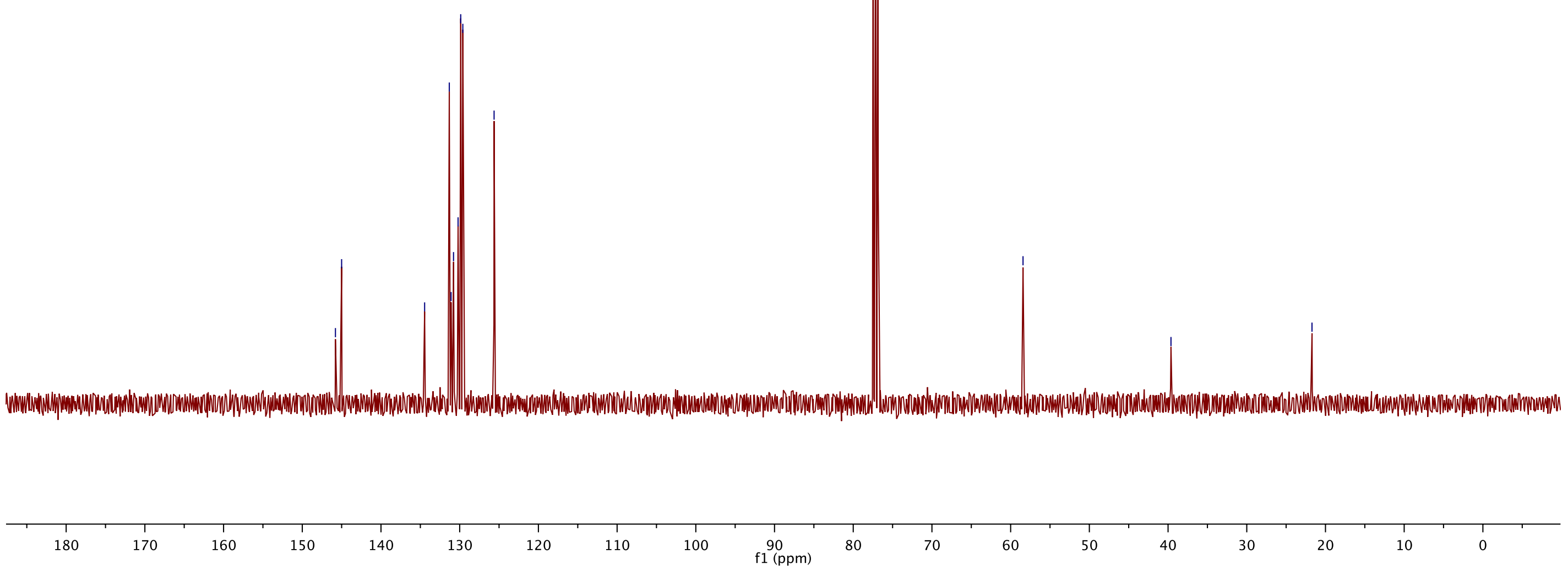

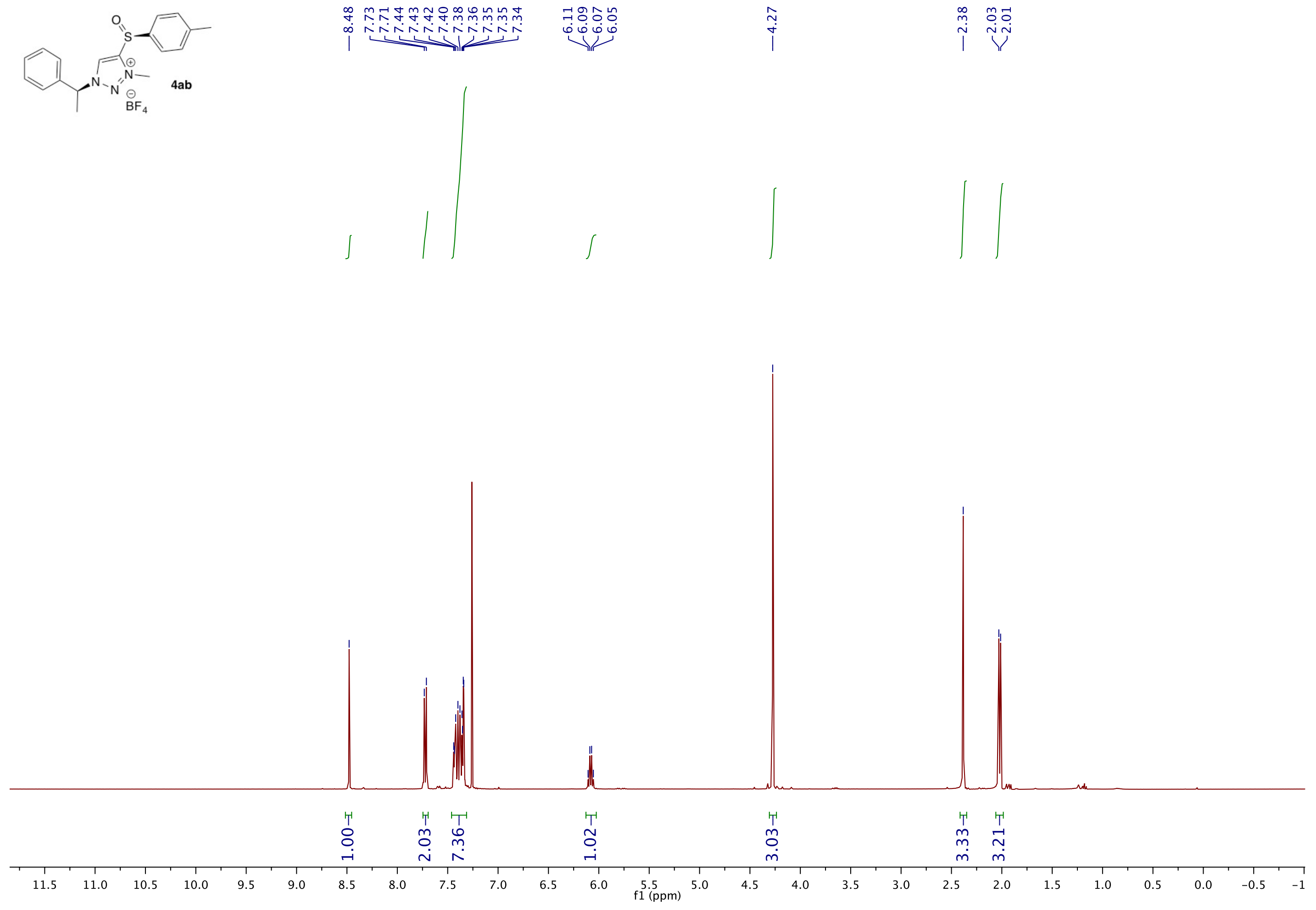

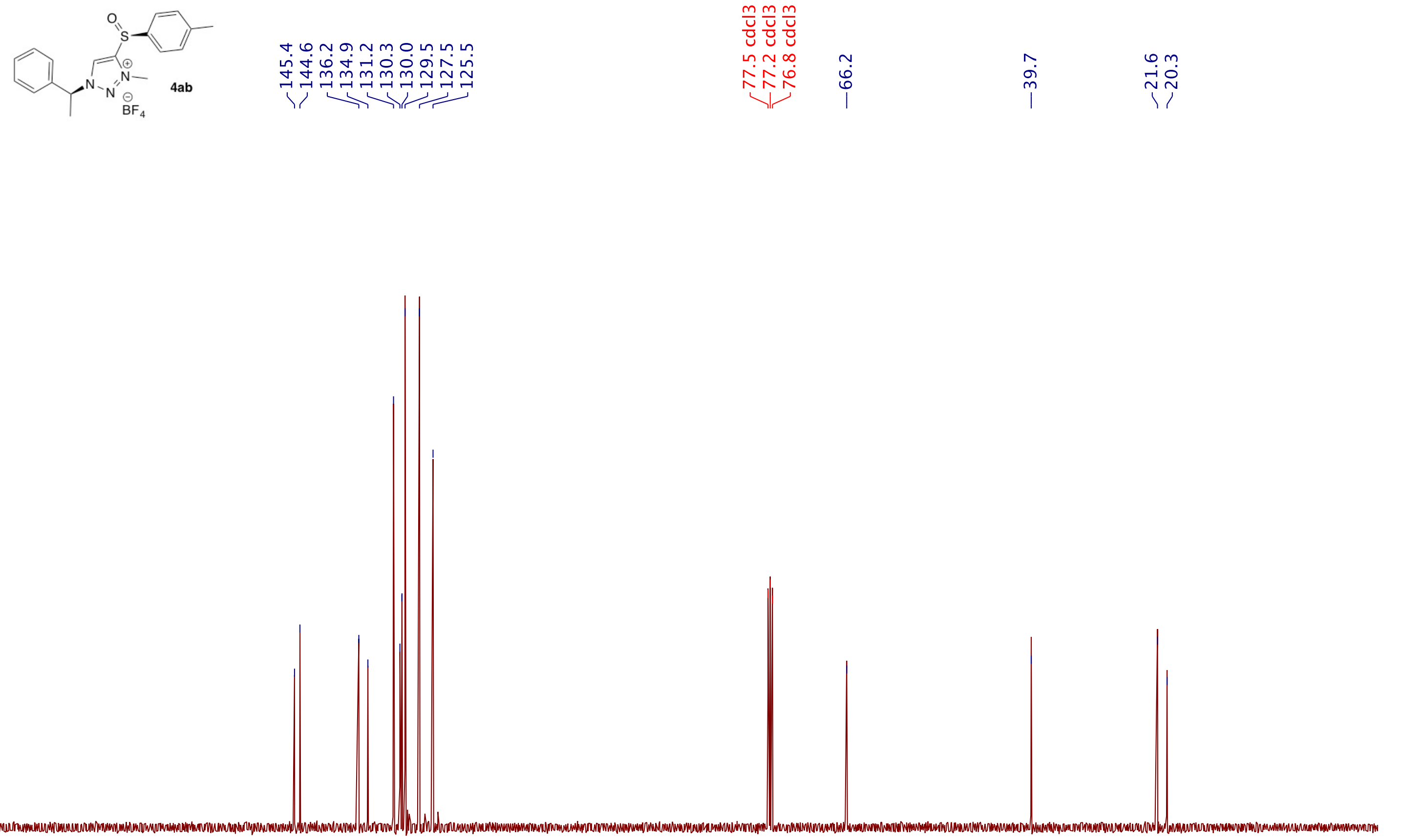

\begin{tabular}{|c|c|c|c|c|c|c|c|c|c|c|c|c|c|c|c|c|c|c|}
\hline 1 & 1 & 1 & 1 & 1 & 1 & 1 & 1 & 1 & 1 & 1 & 1 & 1 & 1 & 1 & 1 & 1 & 1 & $T$ \\
\hline 180 & 170 & 160 & 150 & 140 & 130 & 120 & 110 & 100 & $\begin{array}{c}90 \\
\mathrm{f} 1(\mathrm{ppm})\end{array}$ & 80 & 70 & 60 & 50 & 40 & 30 & 20 & 10 & 0 \\
\hline
\end{tabular}



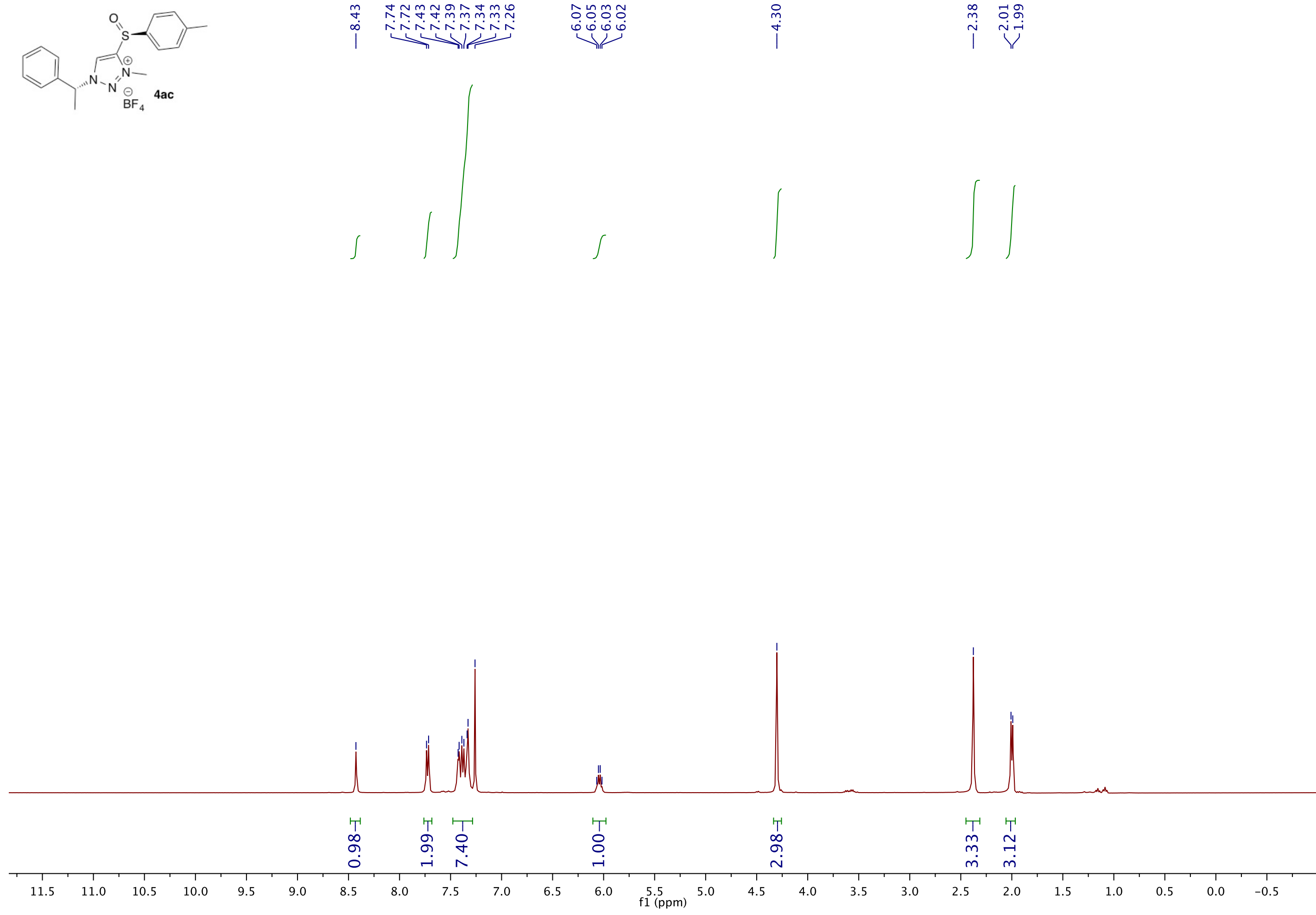


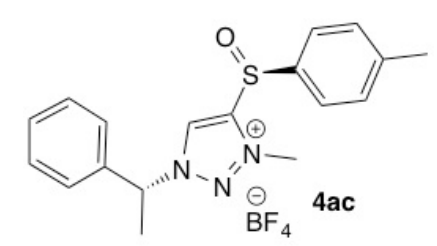

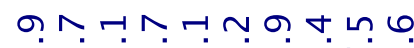

ம்ச்

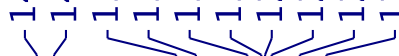

$\mathrm{BF}_{4}$

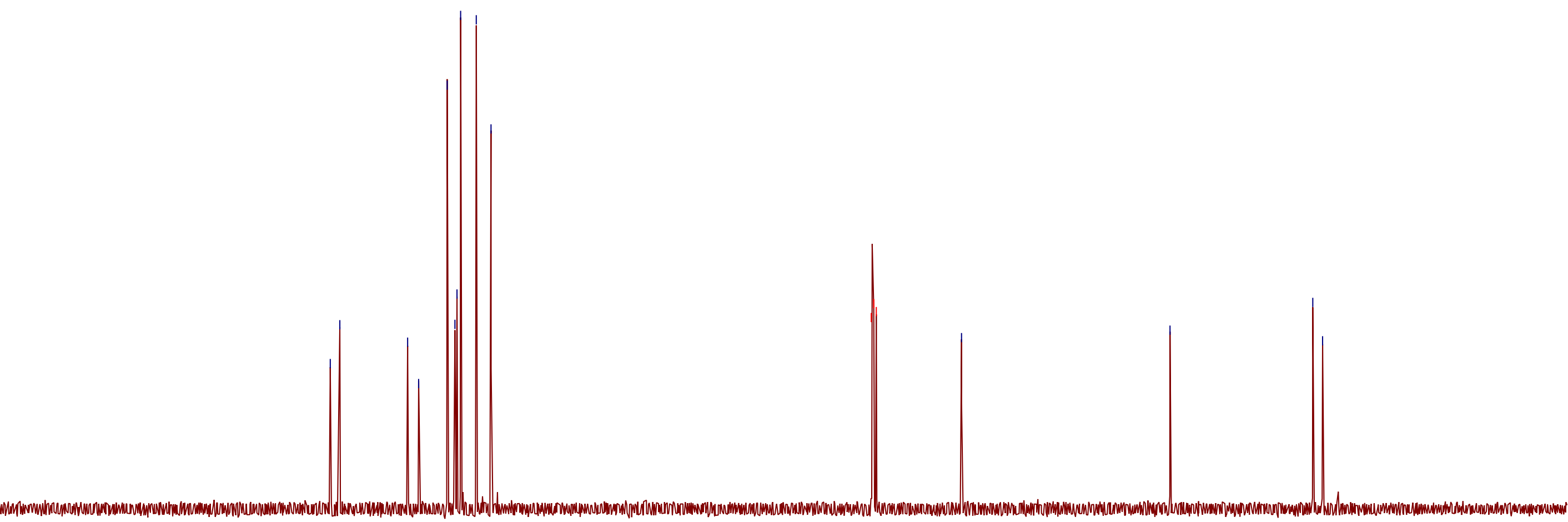

\begin{tabular}{|c|c|c|c|c|c|c|c|c|c|c|c|c|c|c|c|c|c|c|c|}
\hline $\begin{array}{c}1 \\
180\end{array}$ & 170 & $\begin{array}{c}1 \\
160\end{array}$ & 150 & 140 & 130 & 120 & 110 & 100 & 90 & 80 & $\begin{array}{l}1 \\
70\end{array}$ & 60 & 50 & 40 & 11 & 20 & $\frac{1}{10}$ & $\begin{array}{l}1 \\
0\end{array}$ & -1 \\
\hline 180 & 170 & 100 & 150 & 140 & 130 & $1 \angle 0$ & 110 & 100 & $\begin{array}{c}90 \\
\text { f1 (ppm) }\end{array}$ & 80 & 70 & 60 & 50 & 40 & 30 & 20 & 10 & 0 & -1 \\
\hline
\end{tabular}



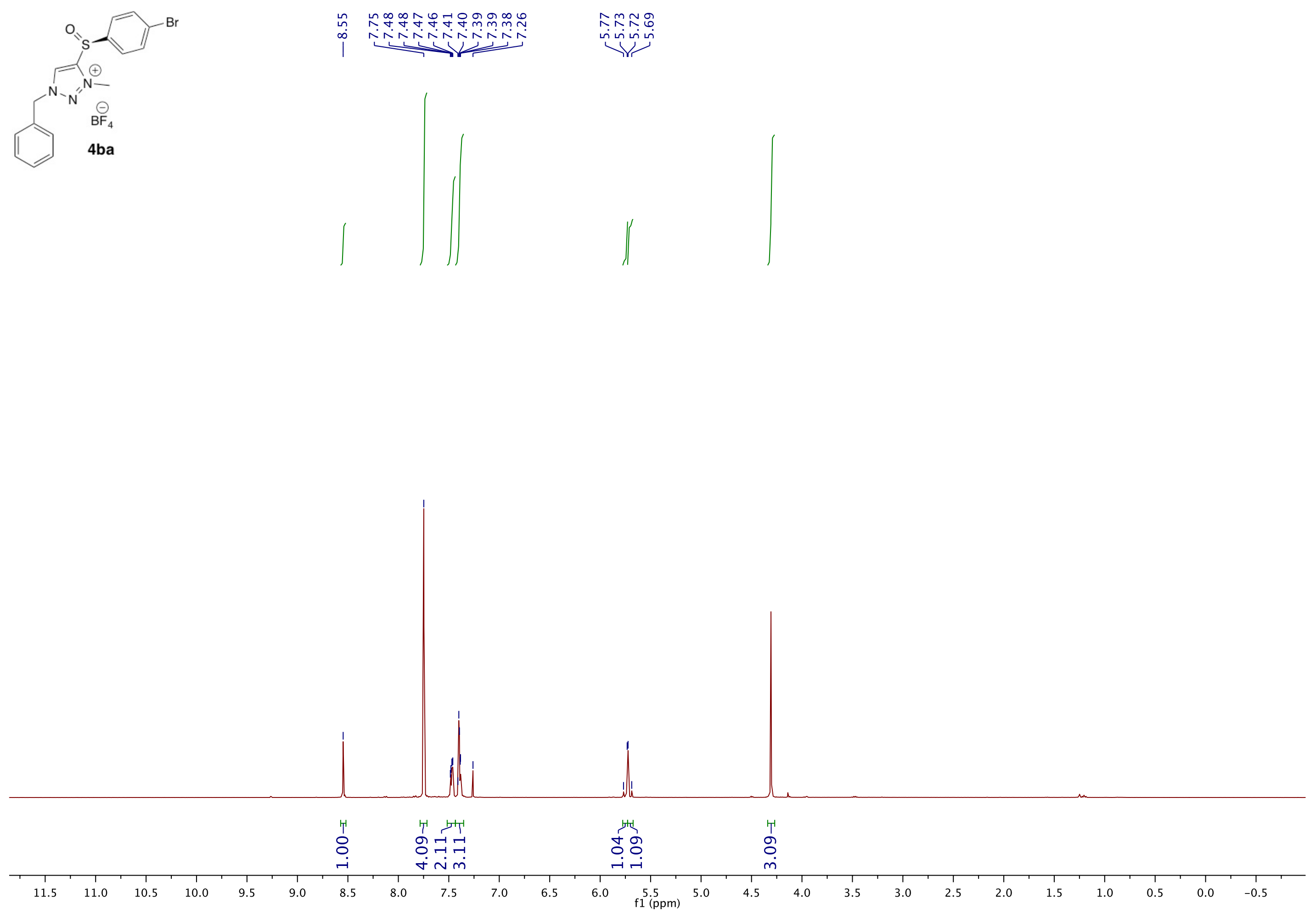


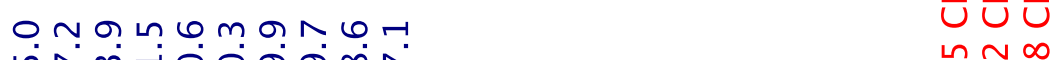

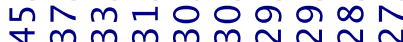

|

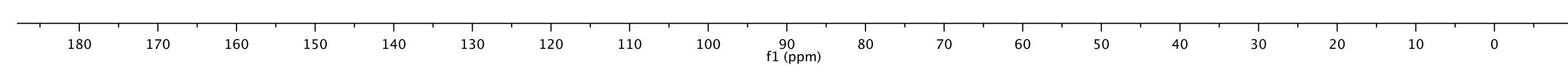



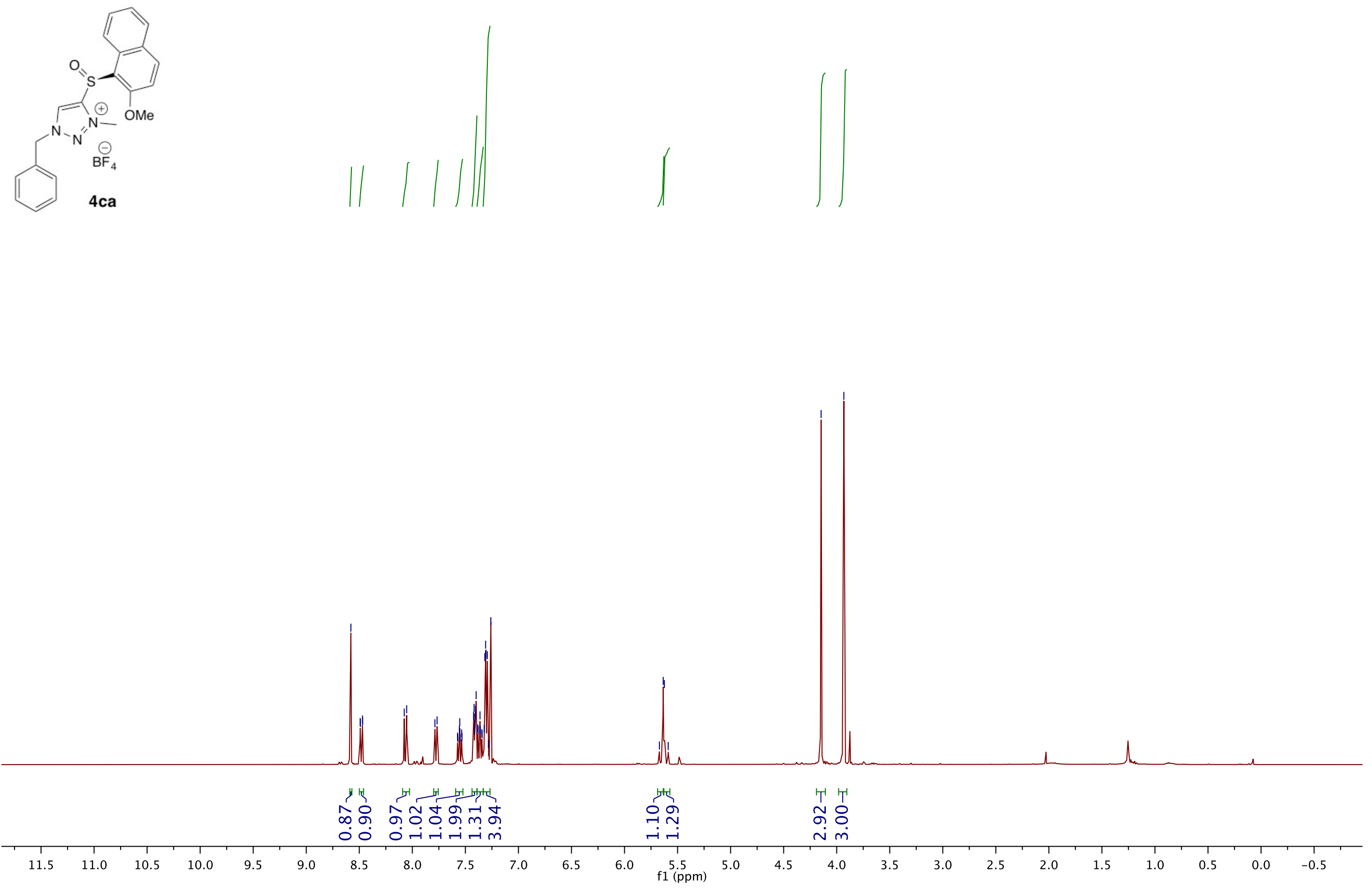


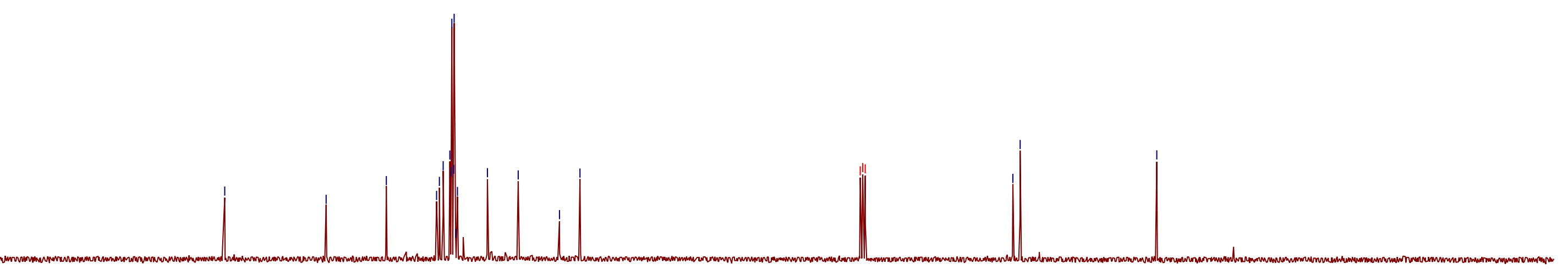

\begin{tabular}{|c|c|c|c|c|c|c|c|c|c|c|c|c|c|c|c|c|c|c|}
\hline $\begin{array}{l}1 \\
180\end{array}$ & 170 & 160 & 150 & $\begin{array}{l}1 \\
140\end{array}$ & $\begin{array}{r}1 \\
130\end{array}$ & $\begin{array}{l}1 \\
120\end{array}$ & 110 & 100 & 90 & $\begin{array}{l}1 \\
80\end{array}$ & 70 & 60 & 50 & 40 & 30 & 20 & 10 & $\begin{array}{r}11 \\
\end{array}$ \\
\hline
\end{tabular}



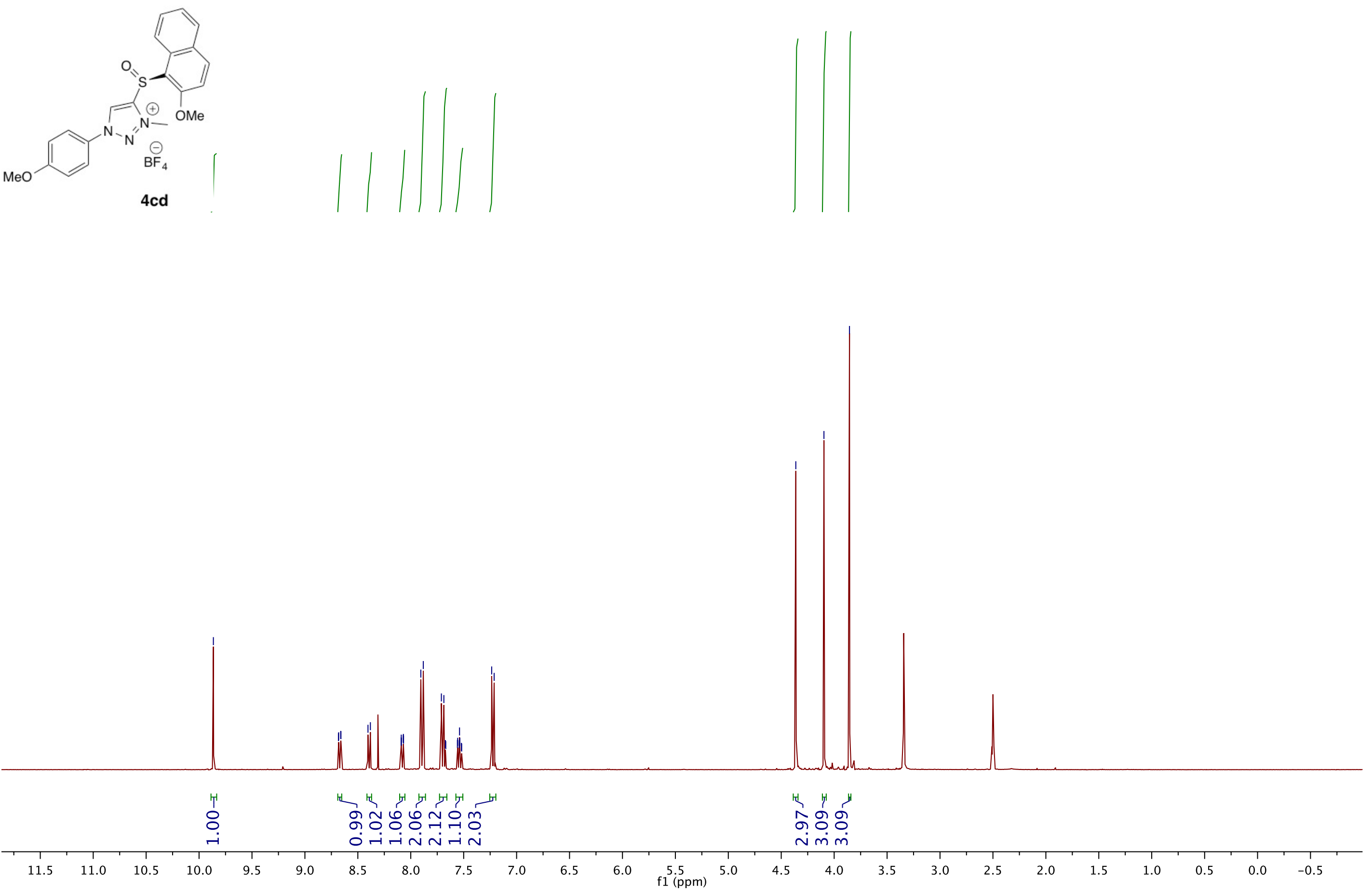


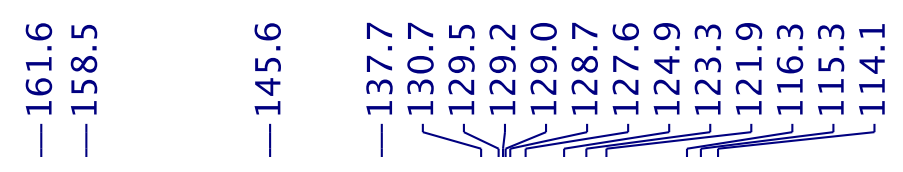

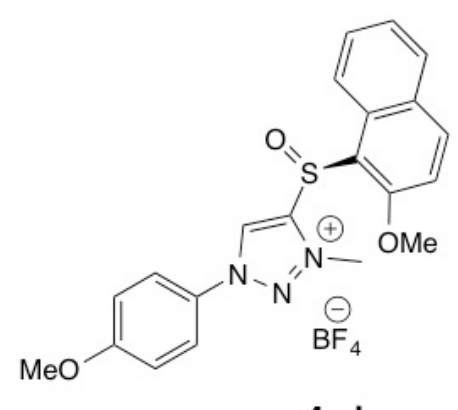

4cd

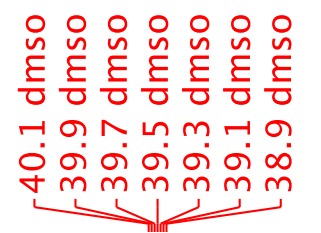

Nं

n

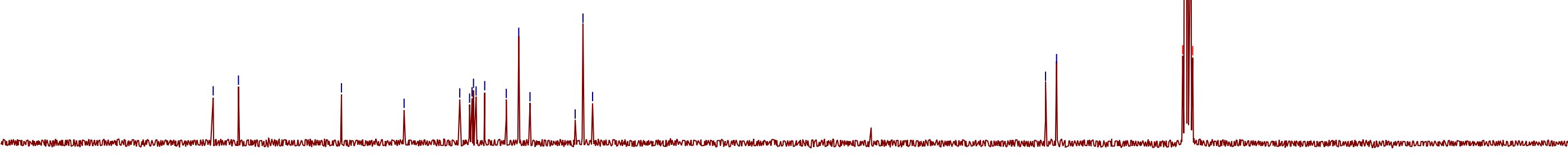


(c)

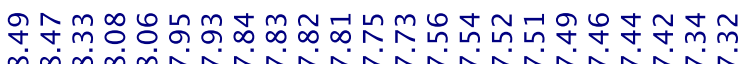
NN公N

$4 \mathrm{ce}$

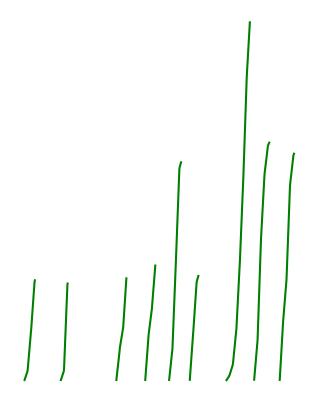

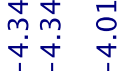

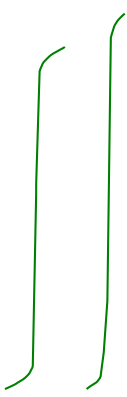

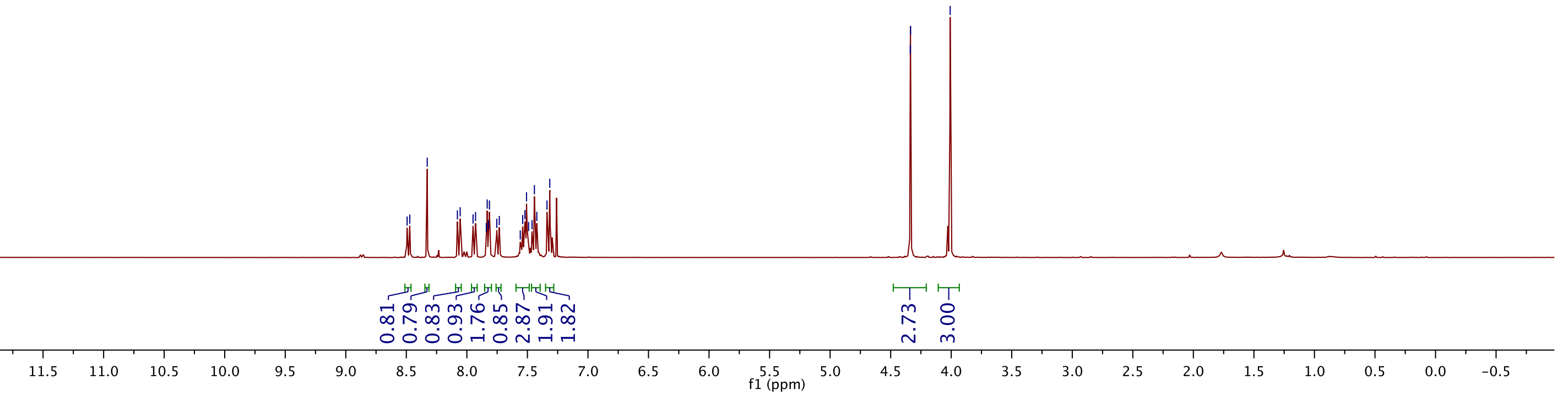




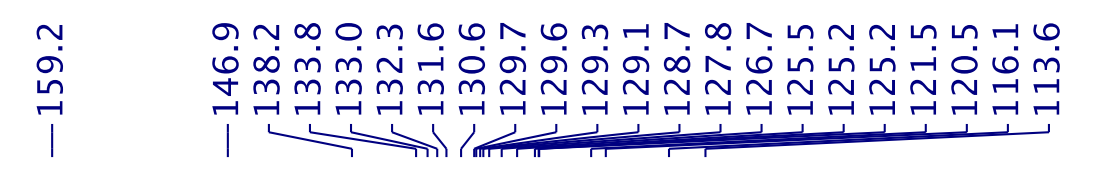

$\frac{m}{v} \frac{m}{v}$

흥 흥

ᄂ $\sim \infty$

ヘ̃ก์

$\stackrel{m}{\stackrel{n}{1}} \quad \stackrel{+}{0}$

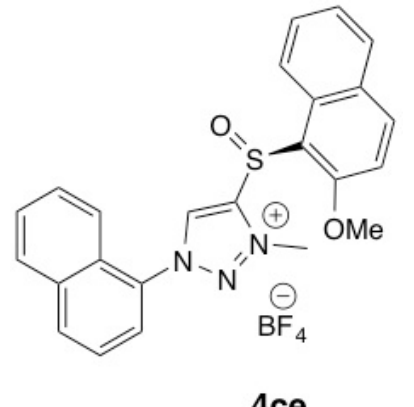

4ce

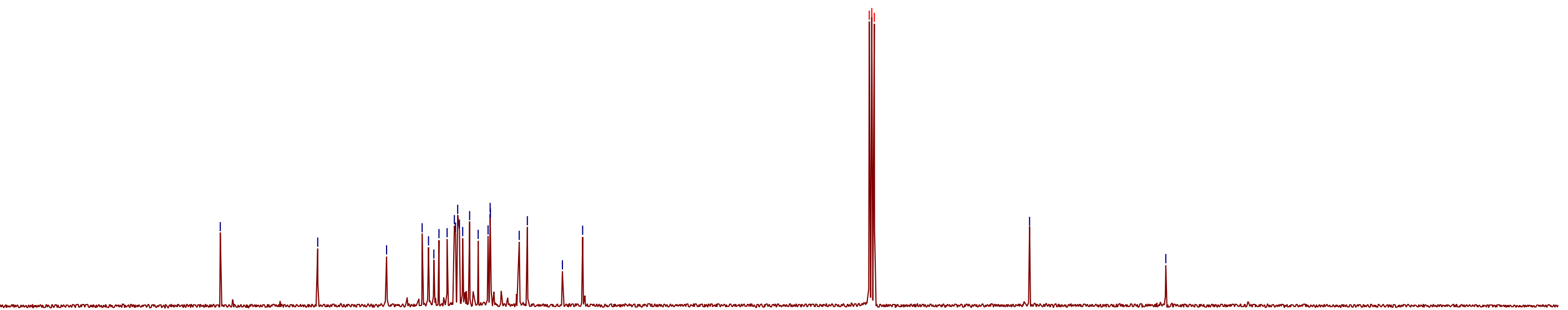

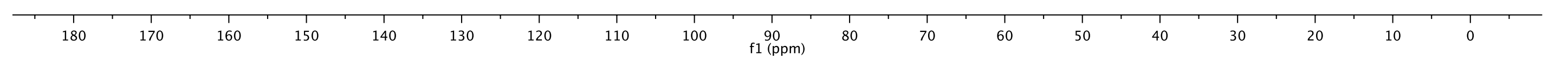



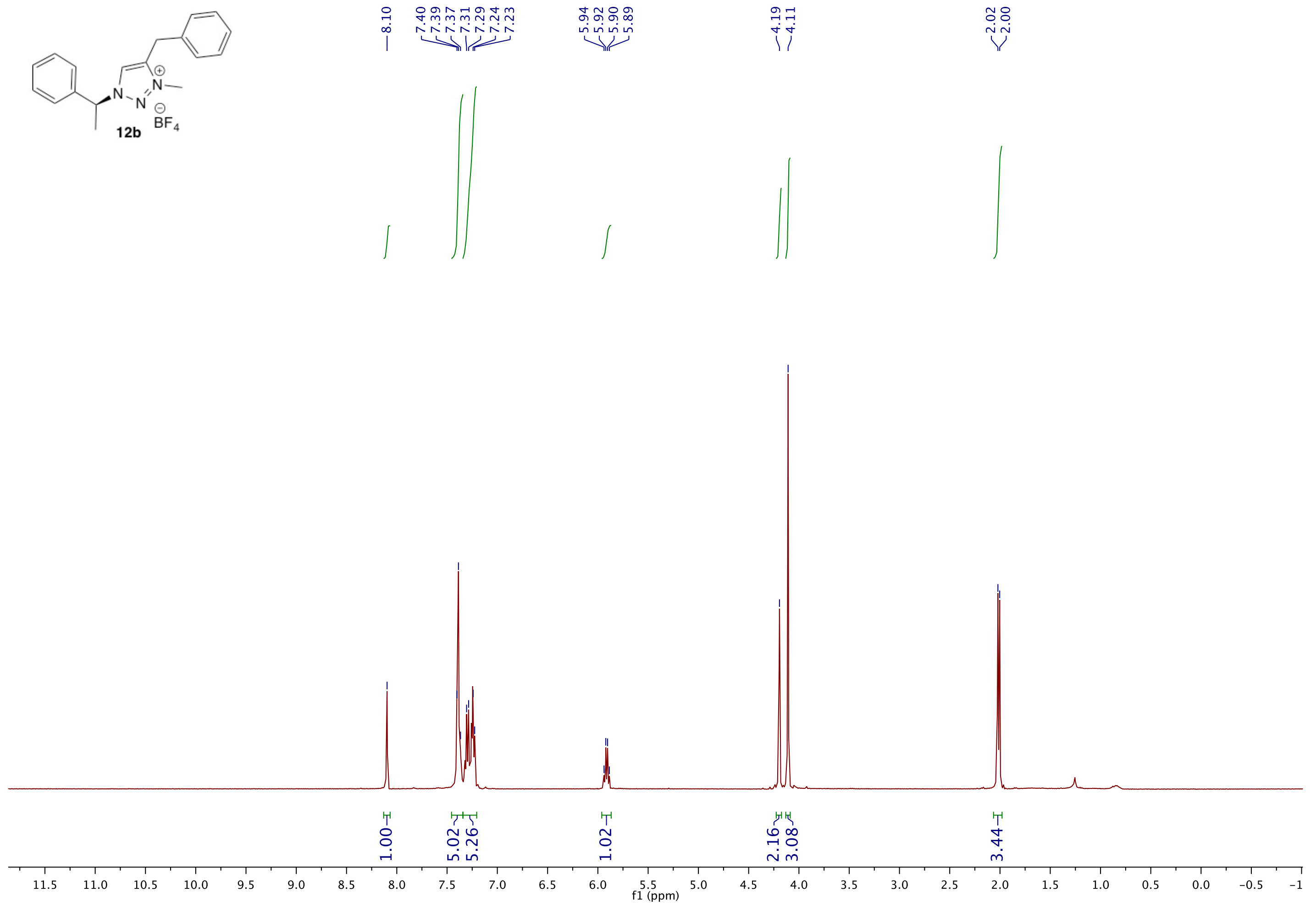
12b $\mathrm{BF}_{4}$

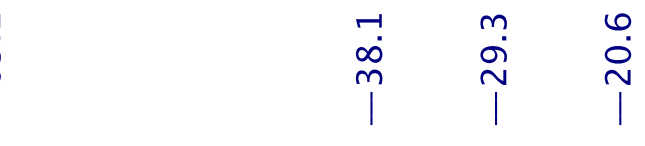

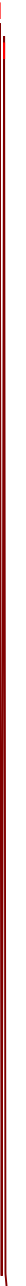

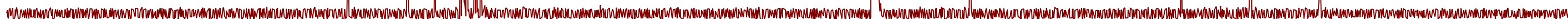



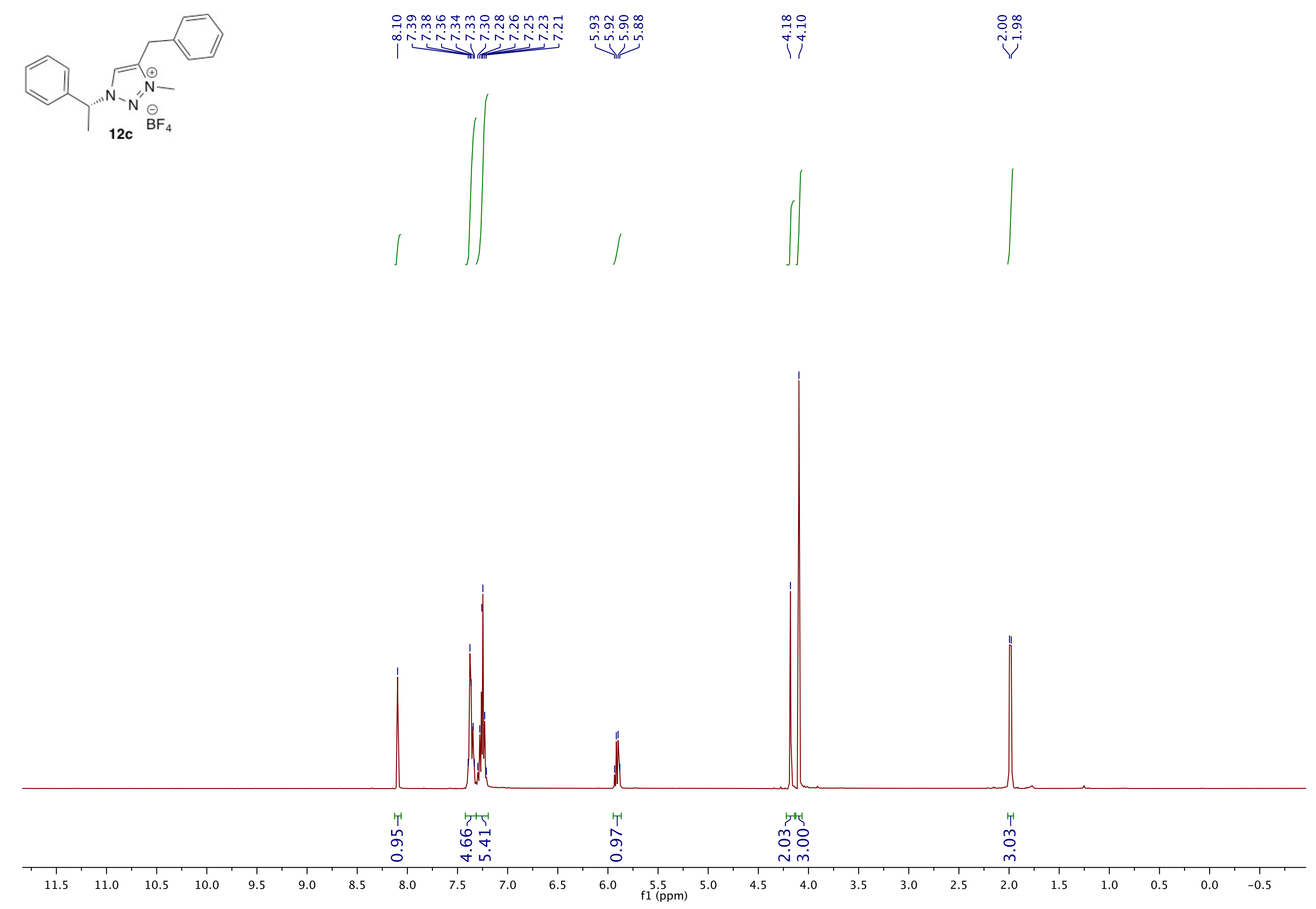


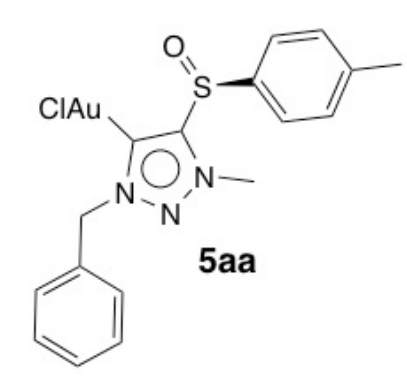

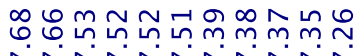

Ln un
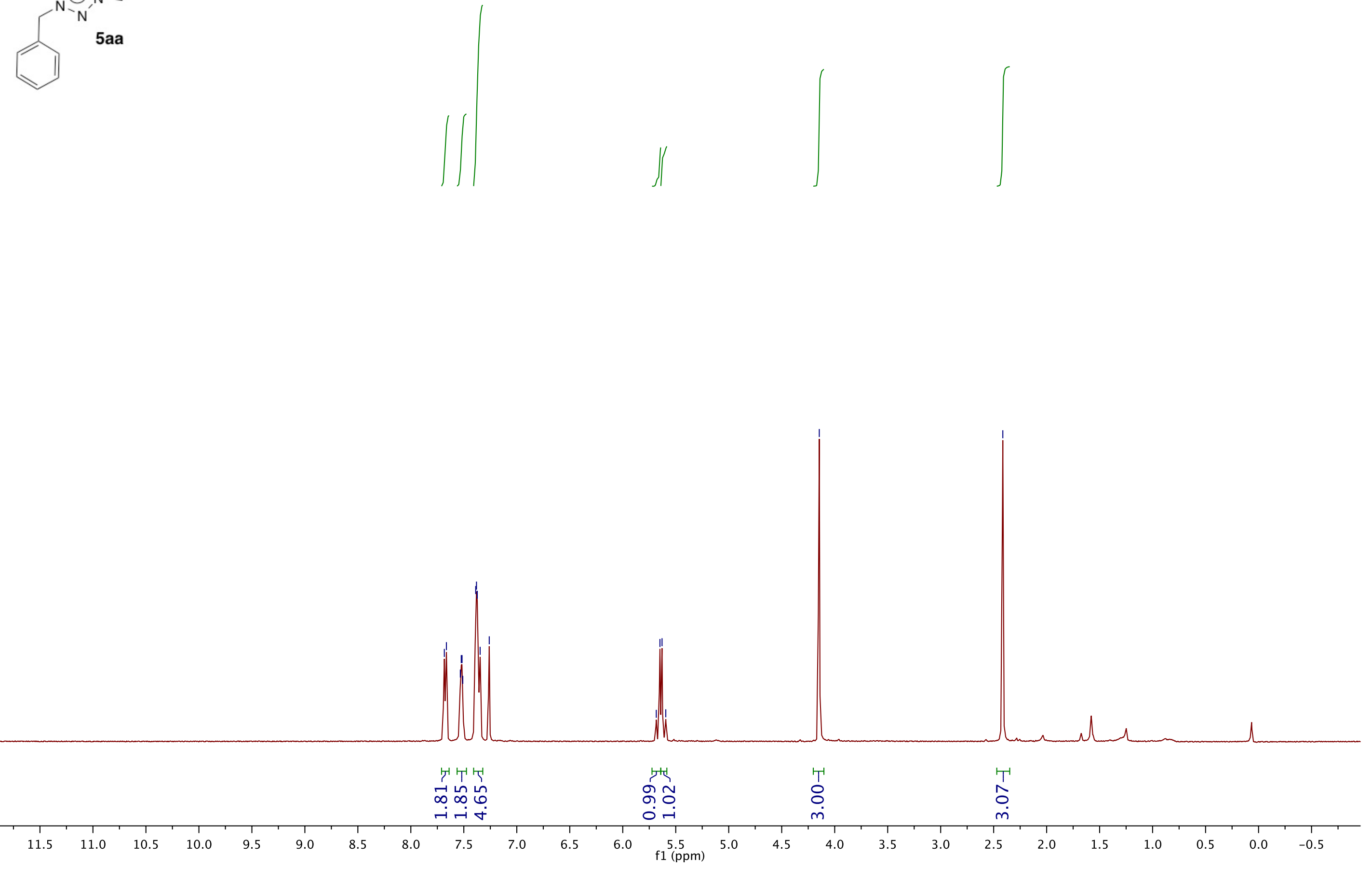

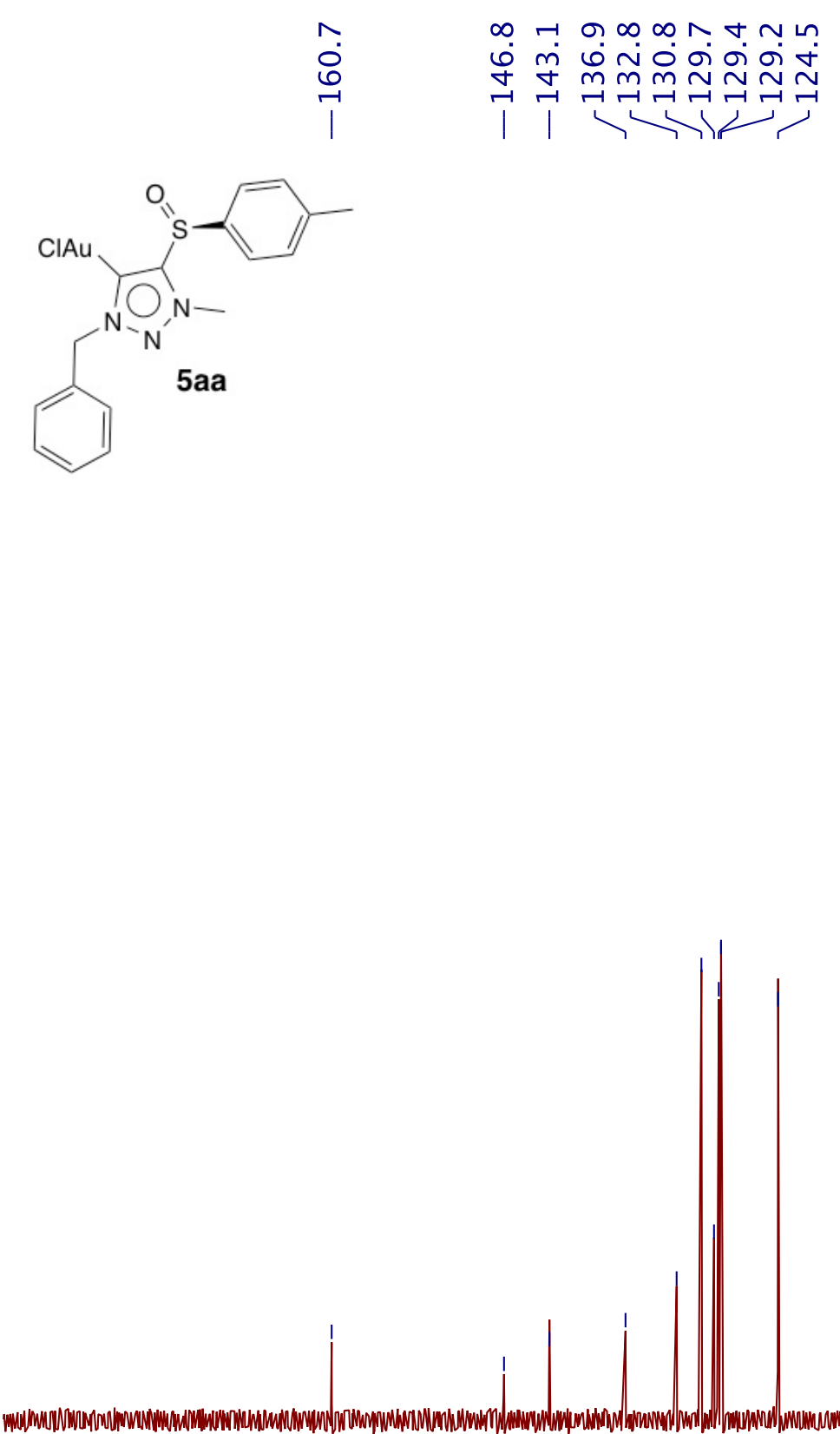

\begin{tabular}{|c|c|c|c|c|c|c|c|c|c|c|c|c|c|c|c|c|c|c|c|}
\hline 11 & 1 & 1 & 1 & 1 & 1 & 1 & 1 & 1 & 11 & 1 & 1 & 1 & 1 & 1 & 1 & 1 & 1 & $T$ & 1 \\
\hline 180 & 170 & 160 & 150 & 140 & 130 & 120 & 110 & 100 & $\begin{array}{l}90 \\
\mathrm{f1}(\mathrm{ppm})\end{array}$ & 80 & 70 & 60 & 50 & 40 & 30 & 20 & 10 & 0 & -10 \\
\hline
\end{tabular}



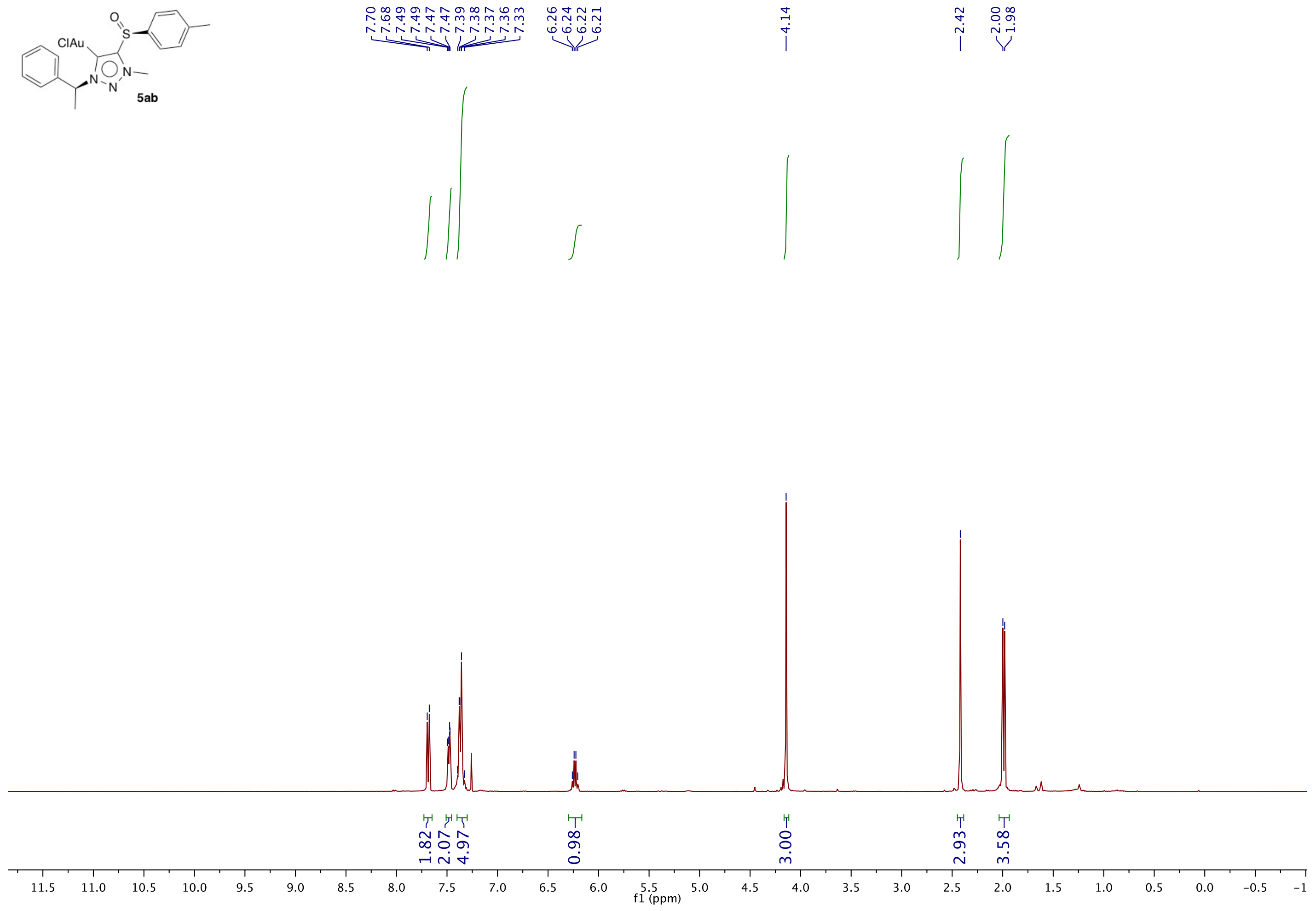

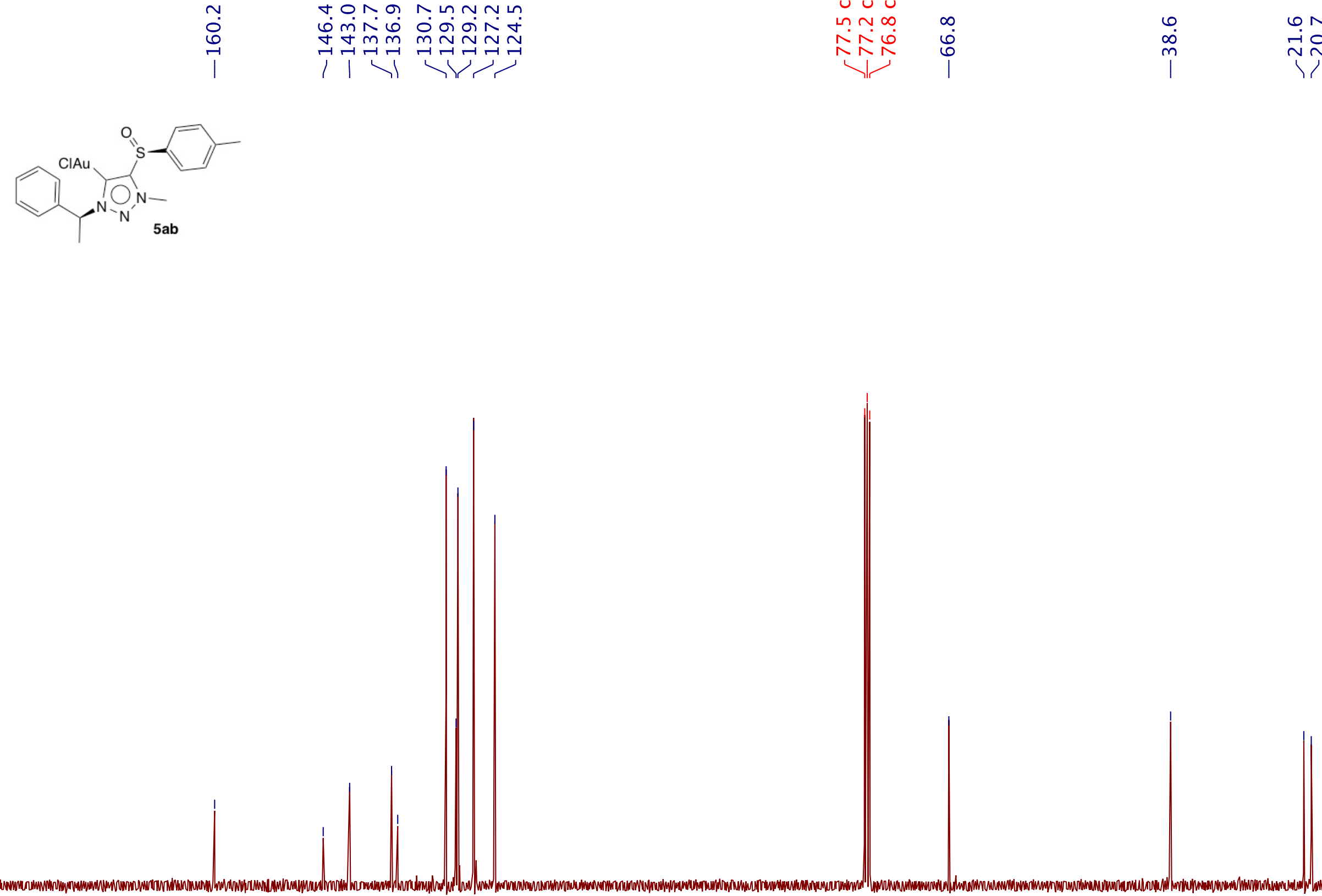

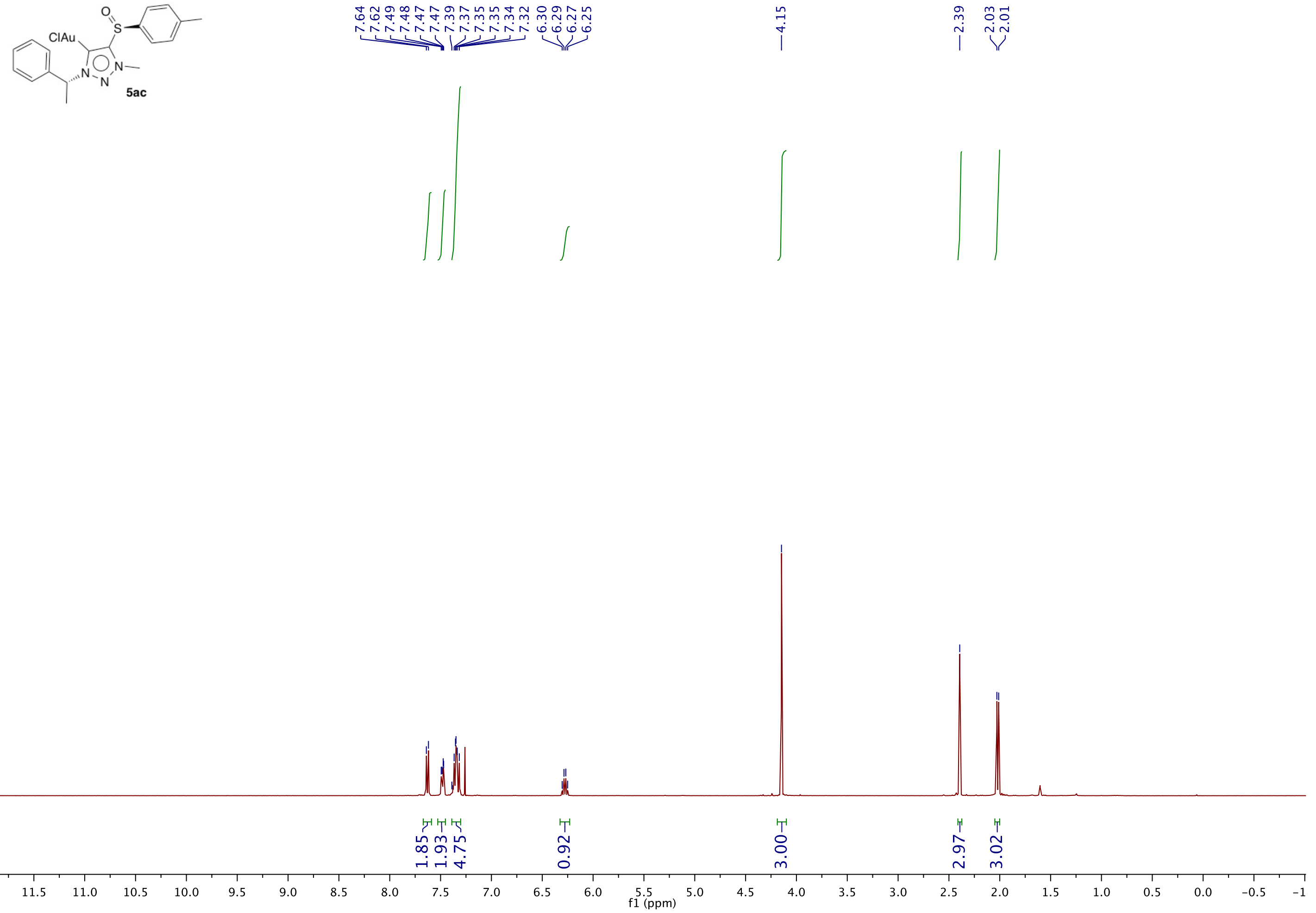

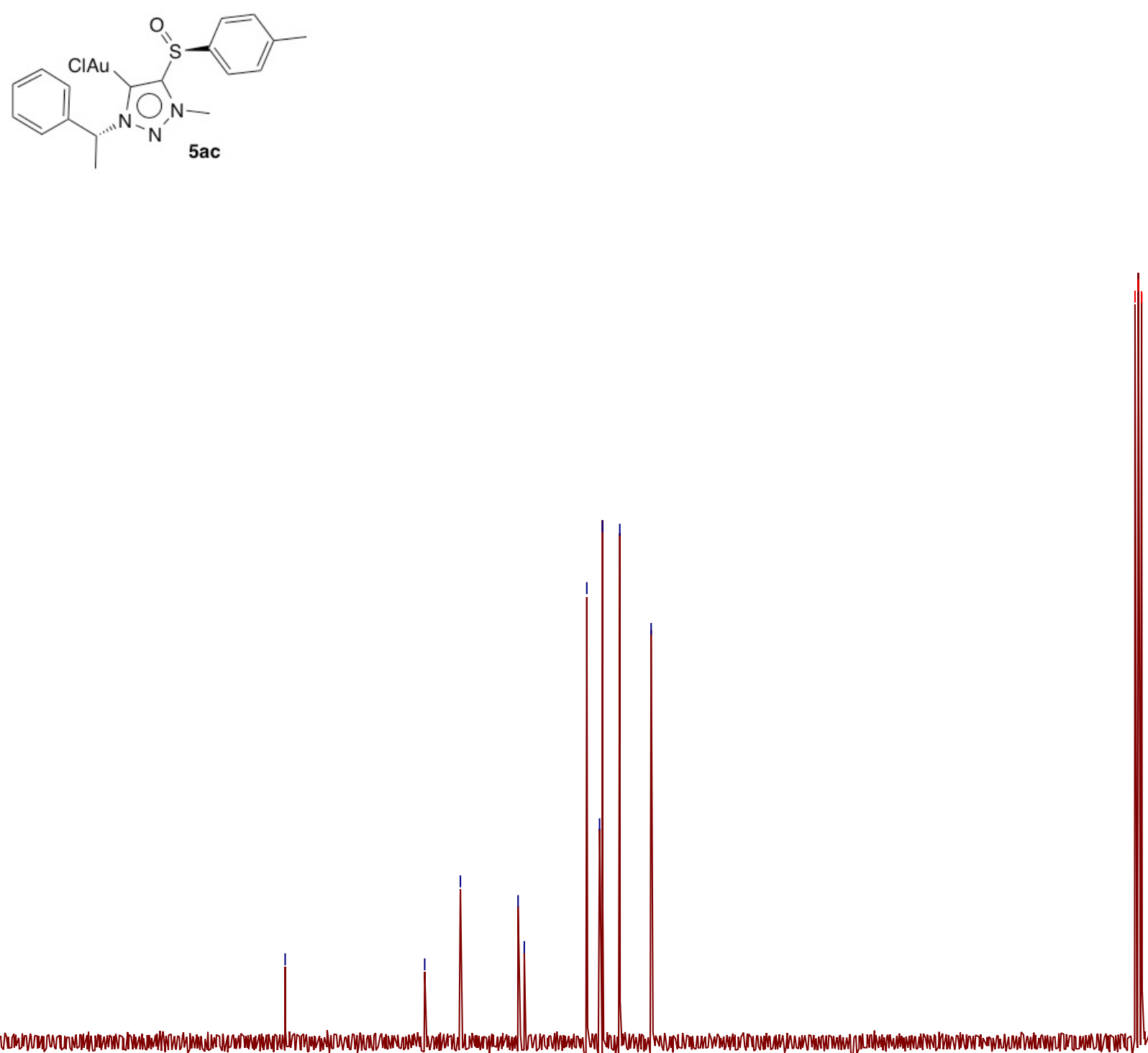

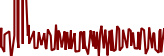

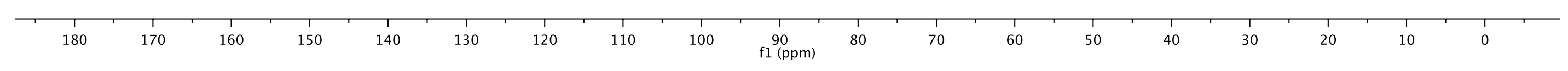



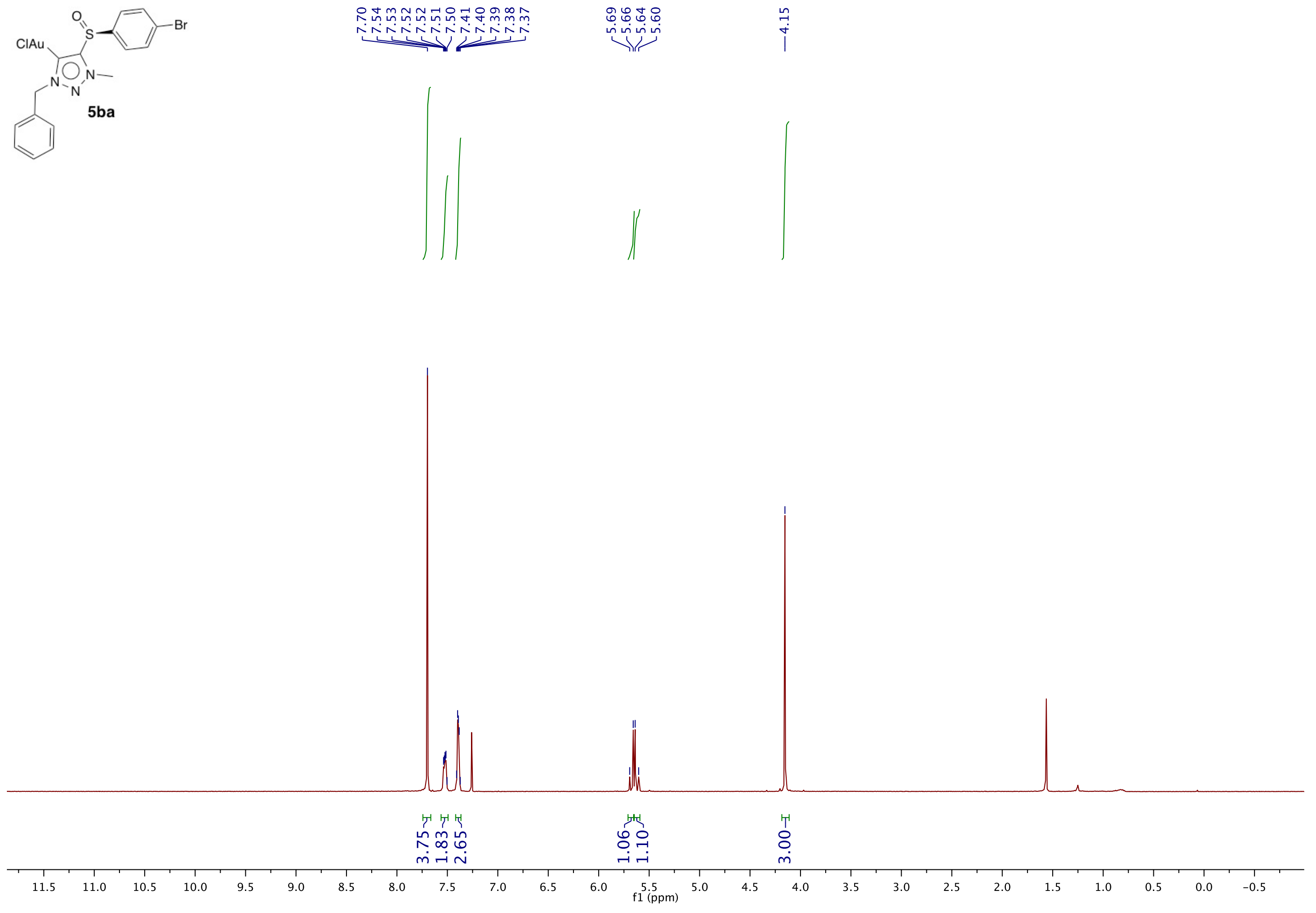

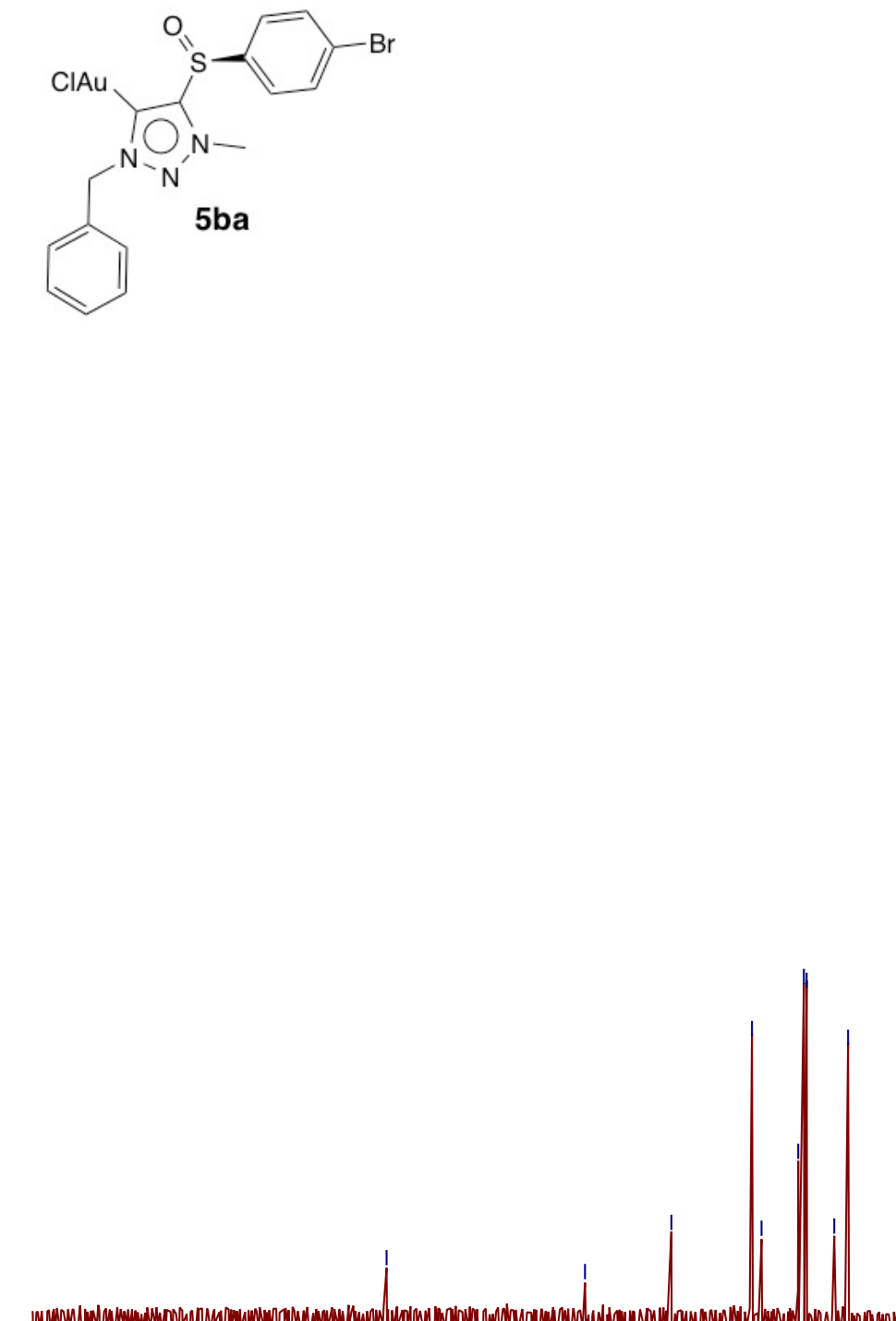

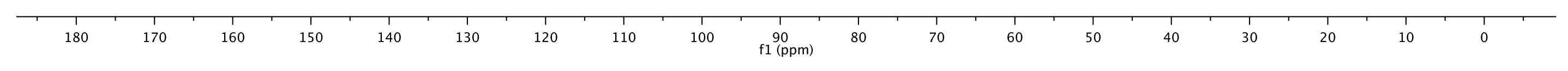



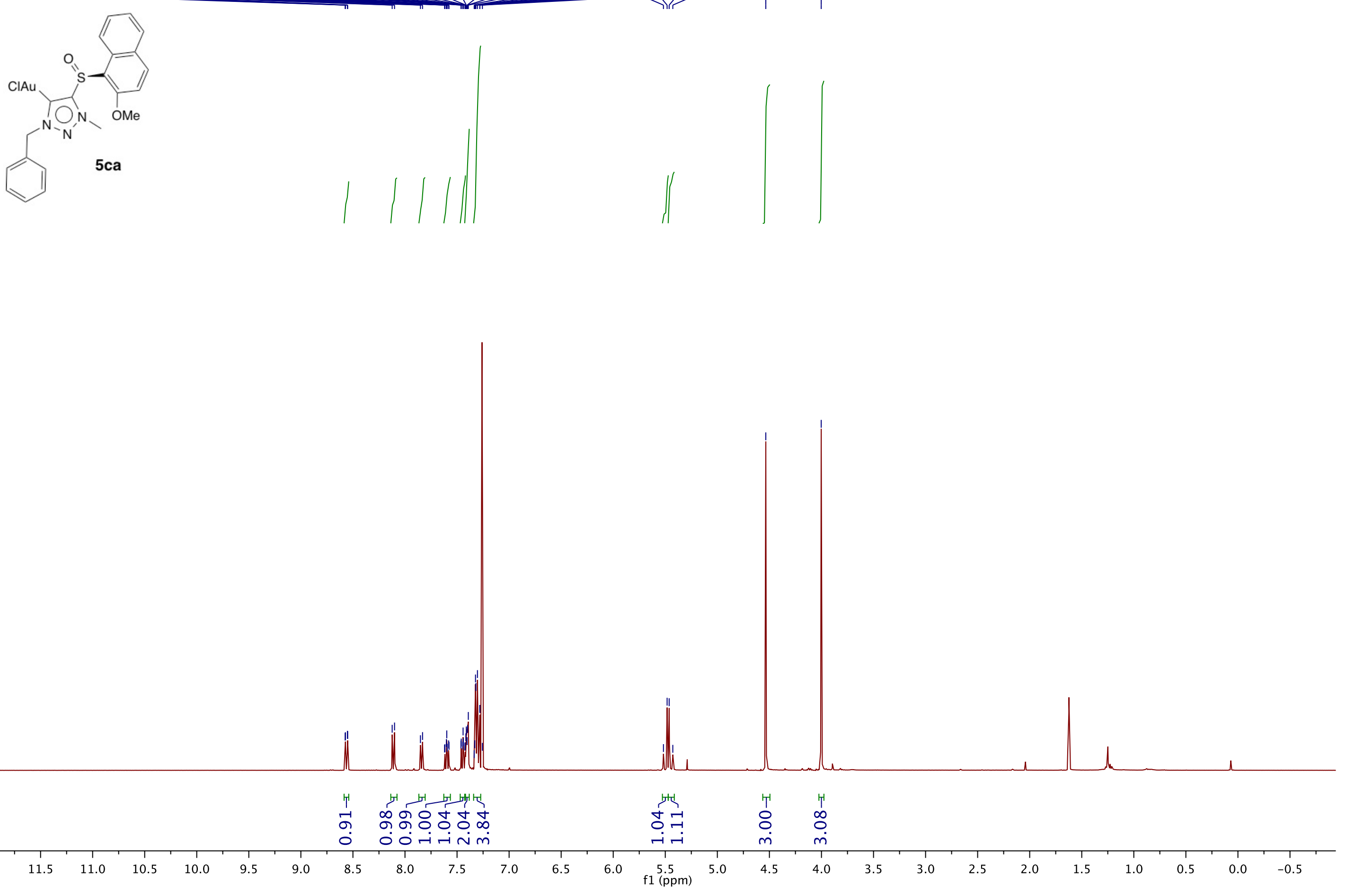


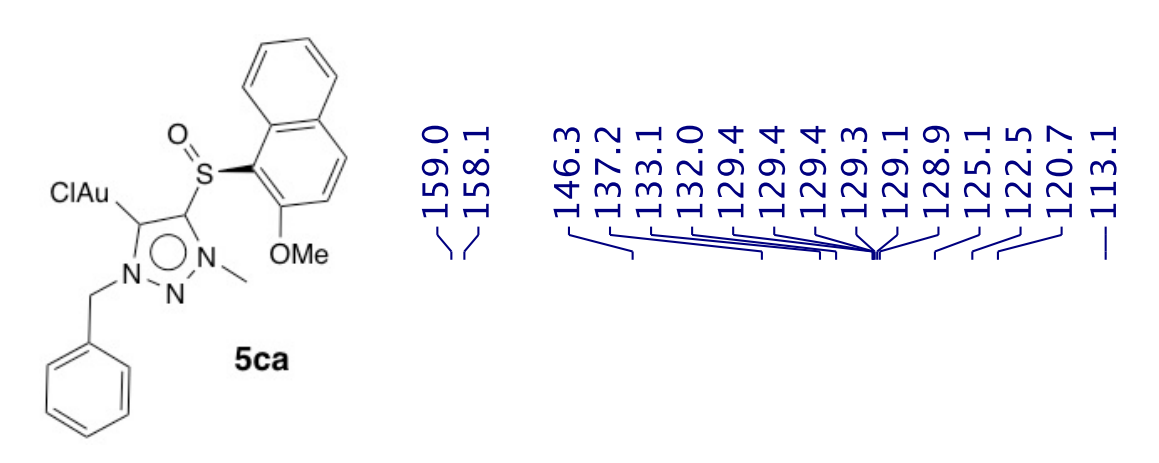

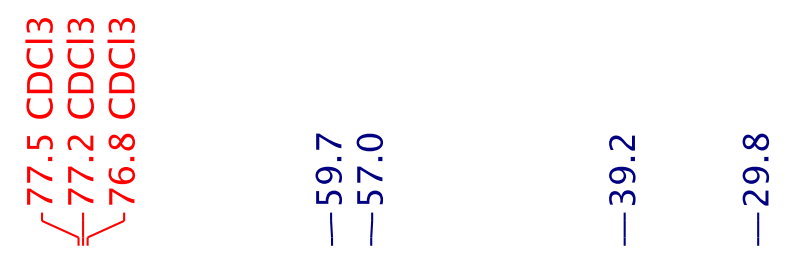

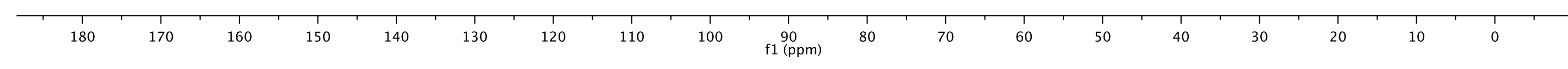



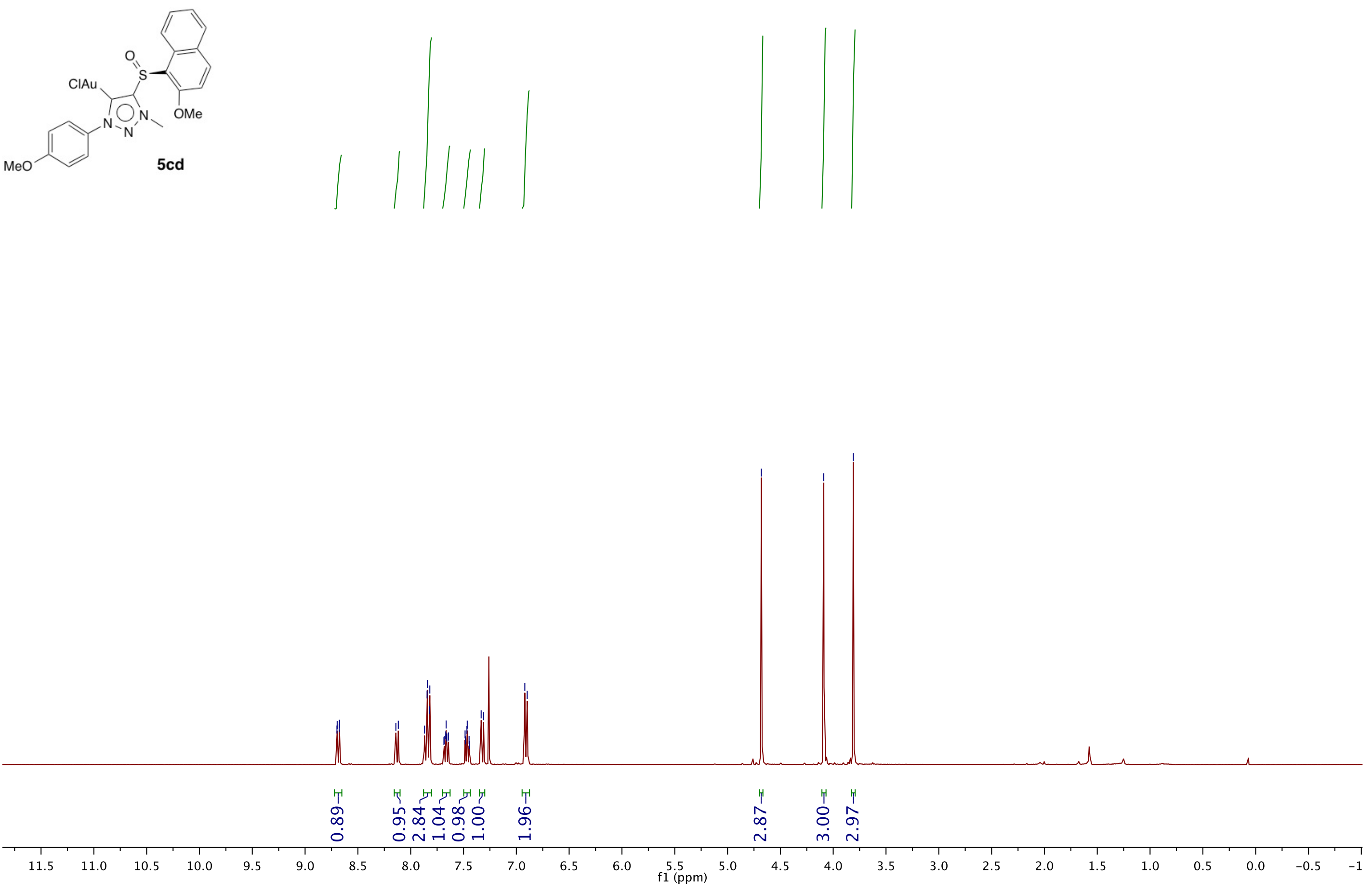
$\sim 0$

تี

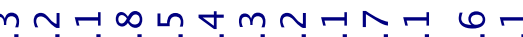

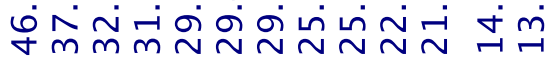

गई

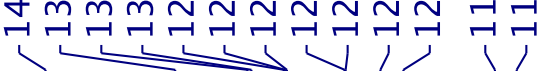

$\mathrm{CIAu}$

$\mathrm{O}$<smiles>c1ccc2c(c1)CCCCC2</smiles>

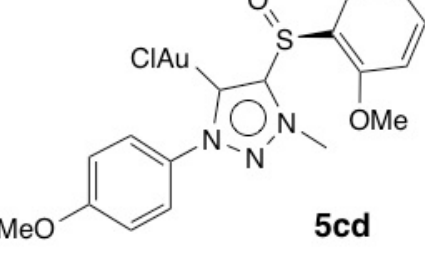

cd

กi

)

\begin{tabular}{|c|c|c|c|c|c|c|c|c|c|c|c|c|c|c|c|c|c|c|c|}
\hline 18 & 17 & 16 & 11 & 1 & 130 & 120 & 110 & 10 & 1 & 80 & 70 & 1 & 11 & 40 & $\begin{array}{l}1 \\
30\end{array}$ & 11 & $\frac{1}{10}$ & $\begin{array}{l}1 \\
0\end{array}$ & $\begin{array}{l}1 \\
-10\end{array}$ \\
\hline 180 & 170 & 160 & 150 & 140 & 130 & 120 & 110 & 100 & $\begin{array}{l}90 \\
\mathrm{f} 1(\mathrm{ppm})\end{array}$ & 80 & 70 & 60 & 50 & 40 & 30 & 20 & 10 & 0 & -10 \\
\hline
\end{tabular}




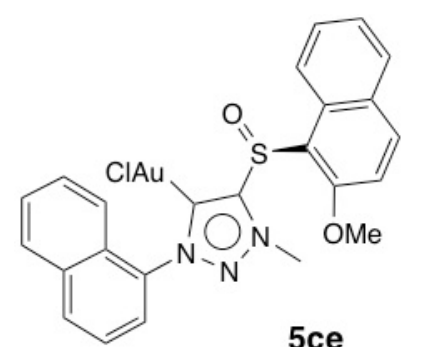

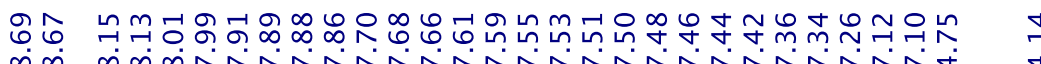

)

5 ce
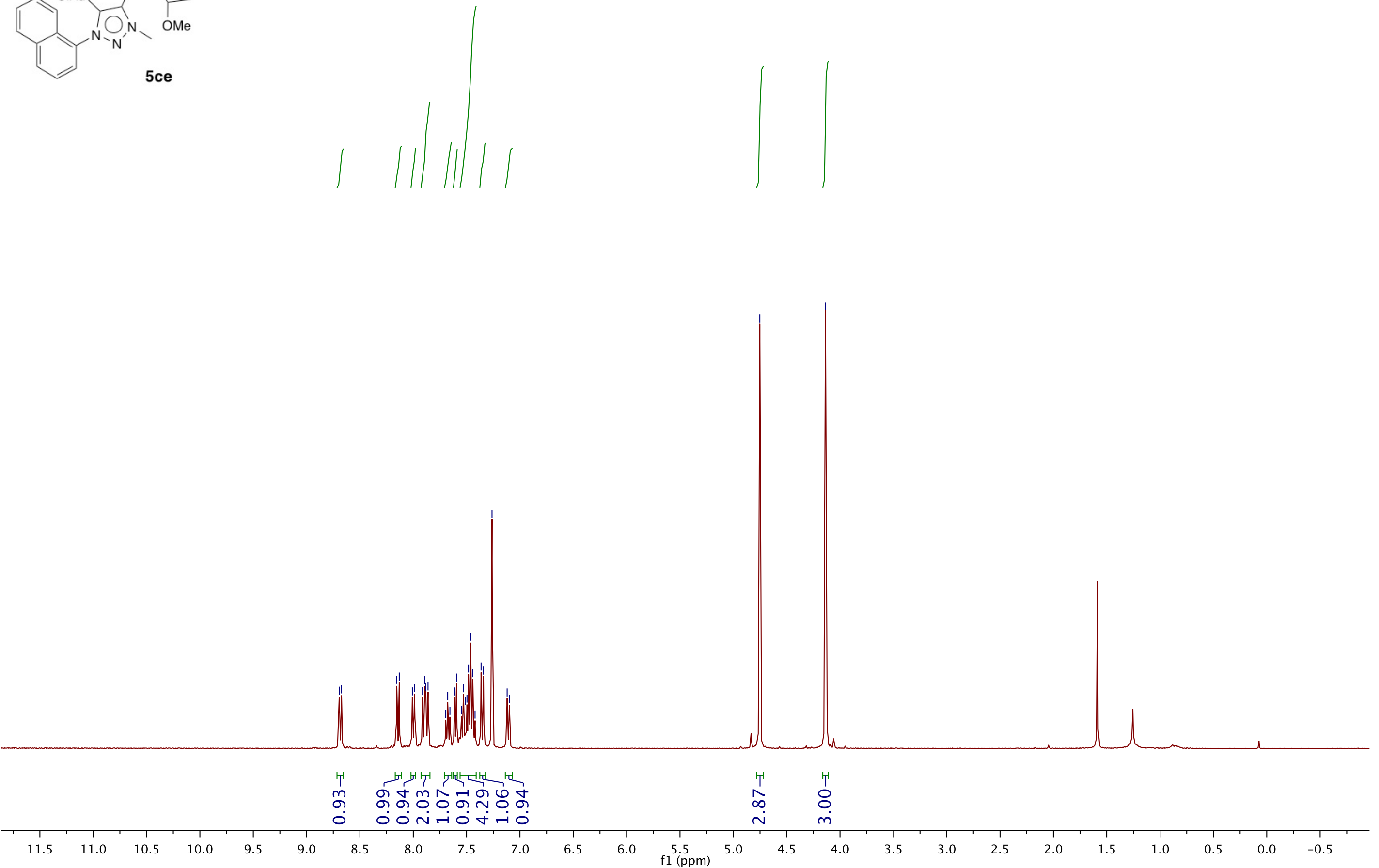


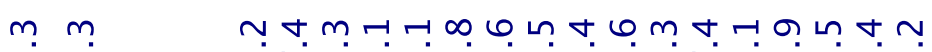

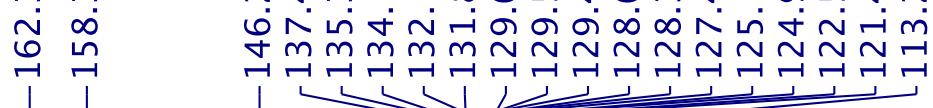

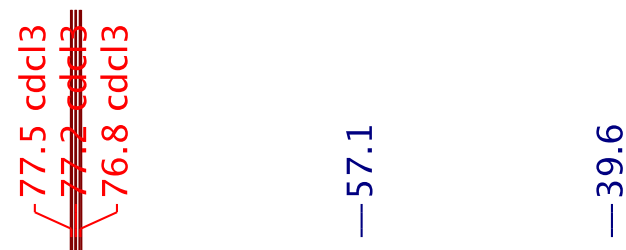
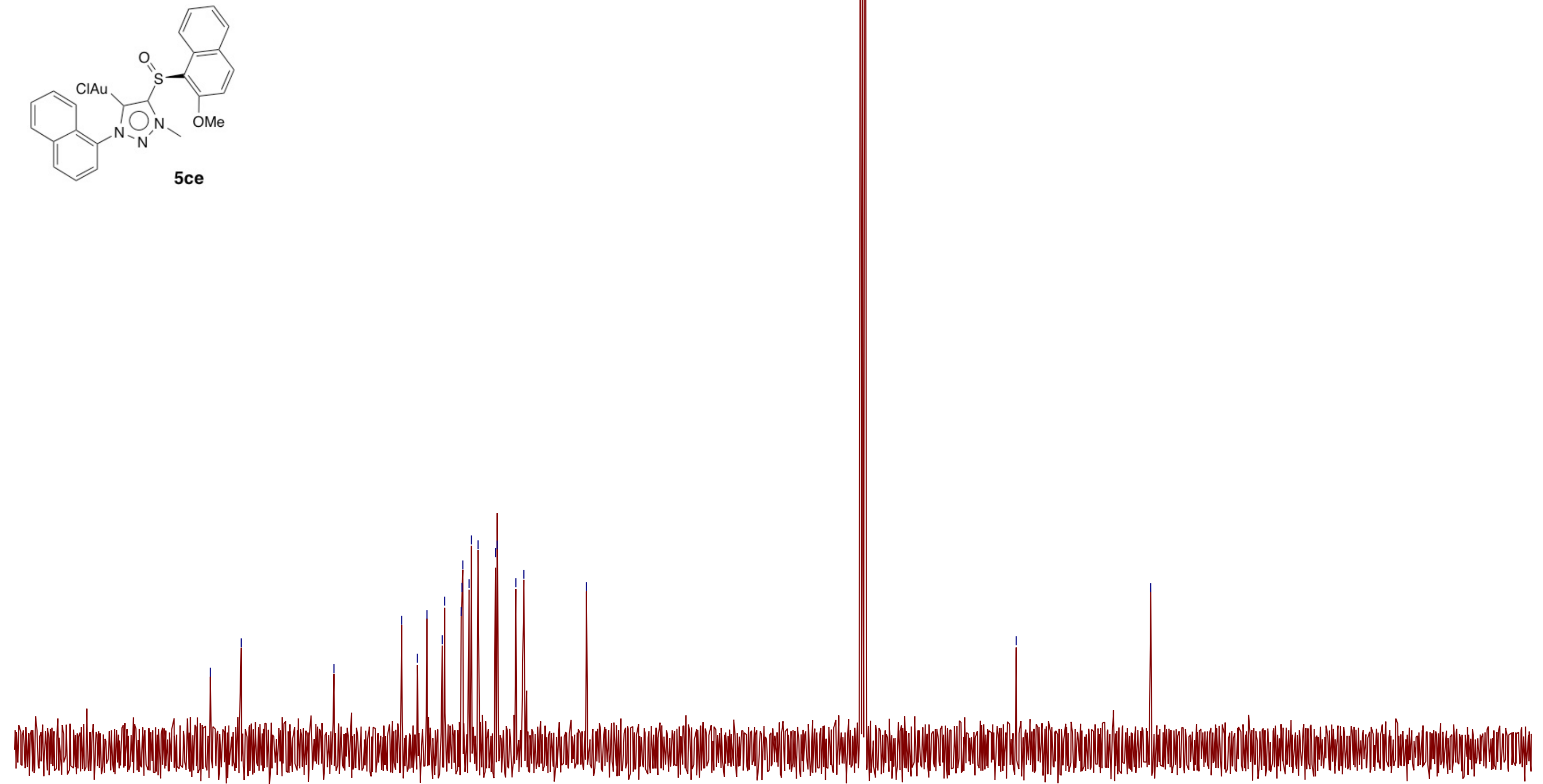

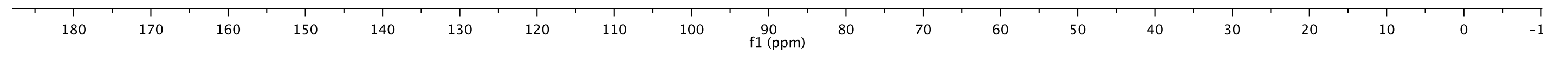




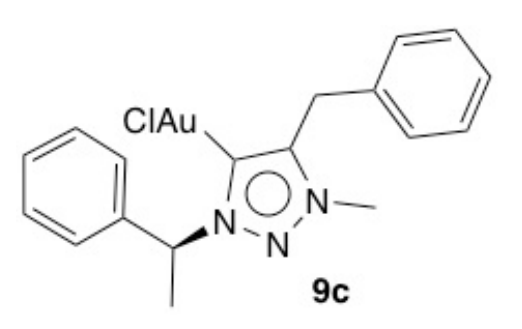

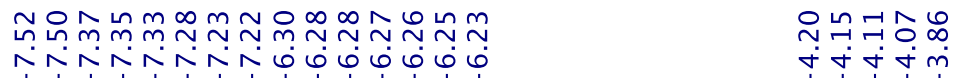

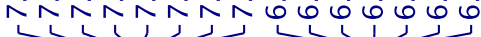

îj
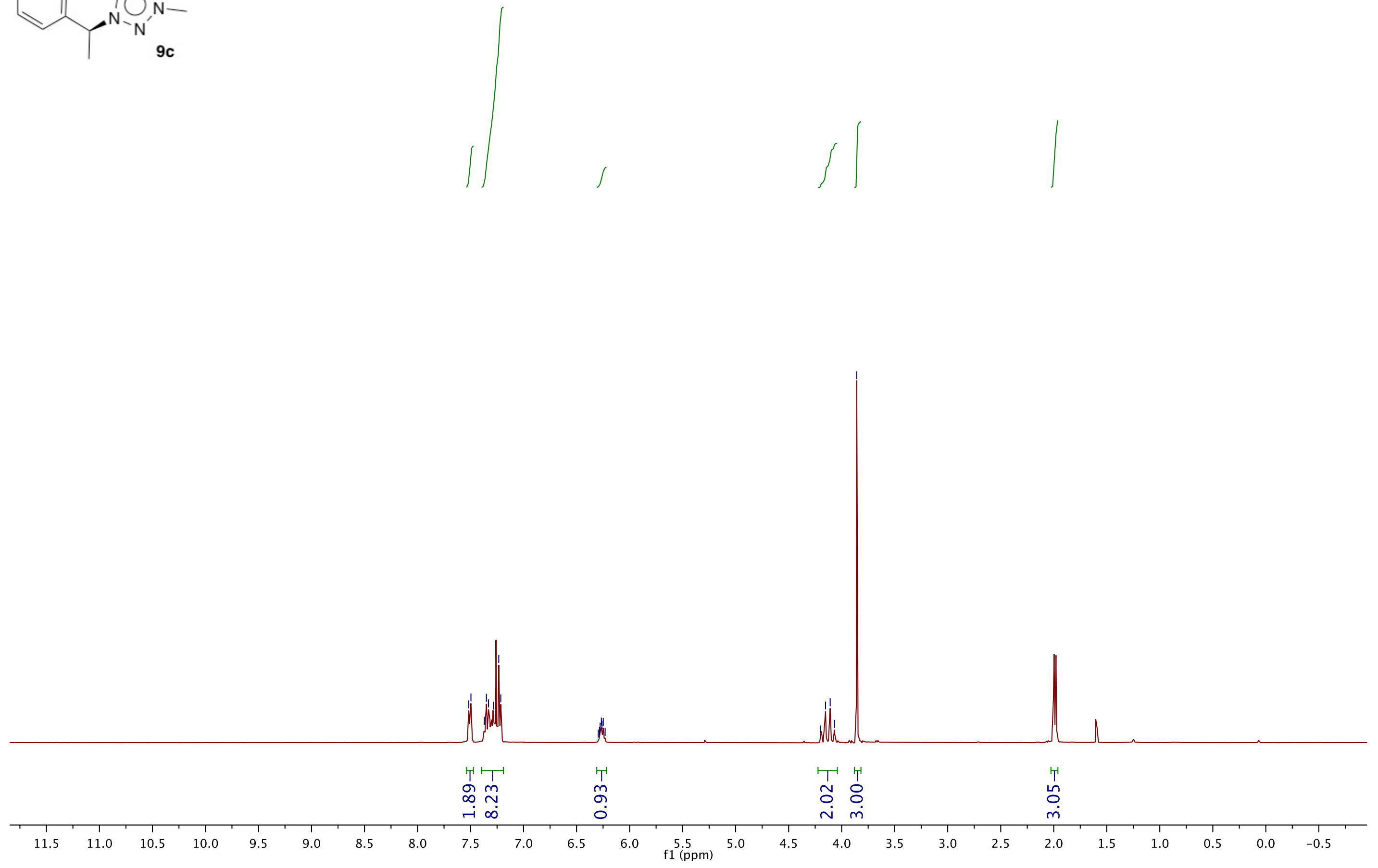
No

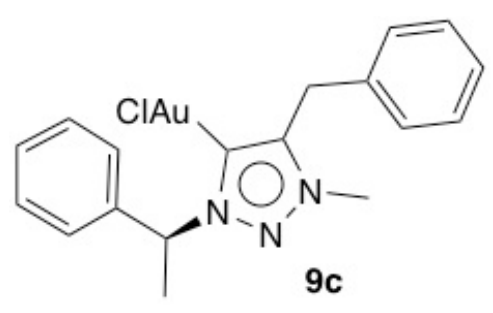

$\frac{m}{v} \frac{m}{v} \frac{m}{v}$

ర

ก $\sim \infty$

ก̃ก

$\stackrel{+}{\circ}$

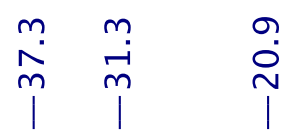

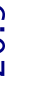

\section{.}



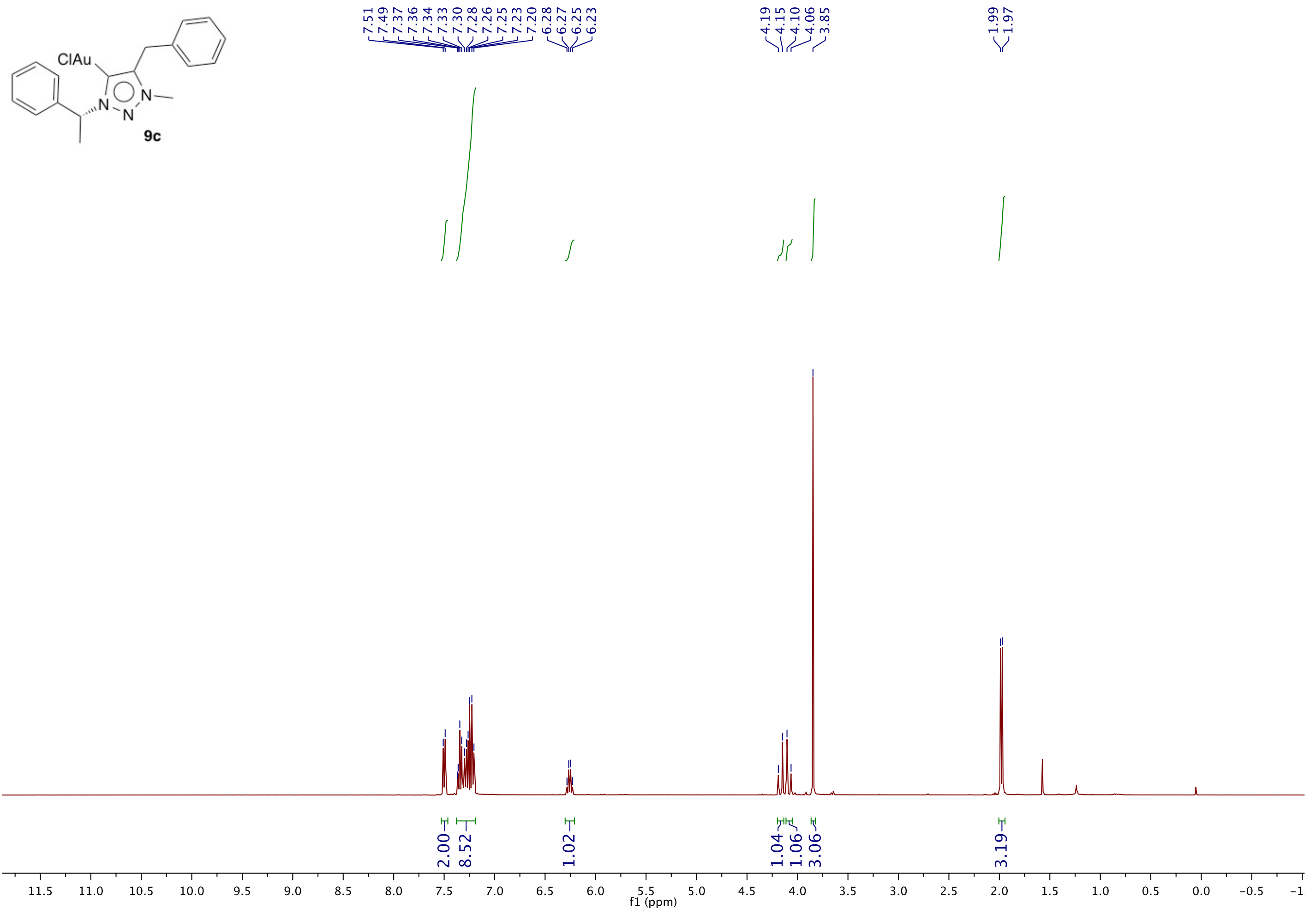

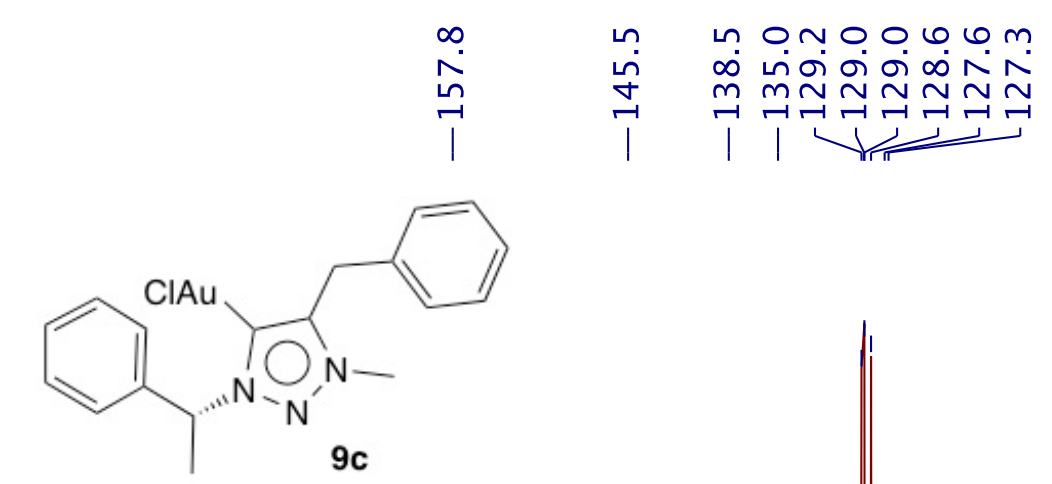

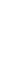

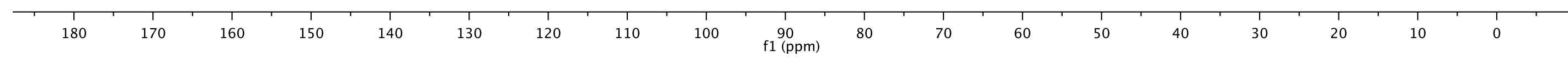



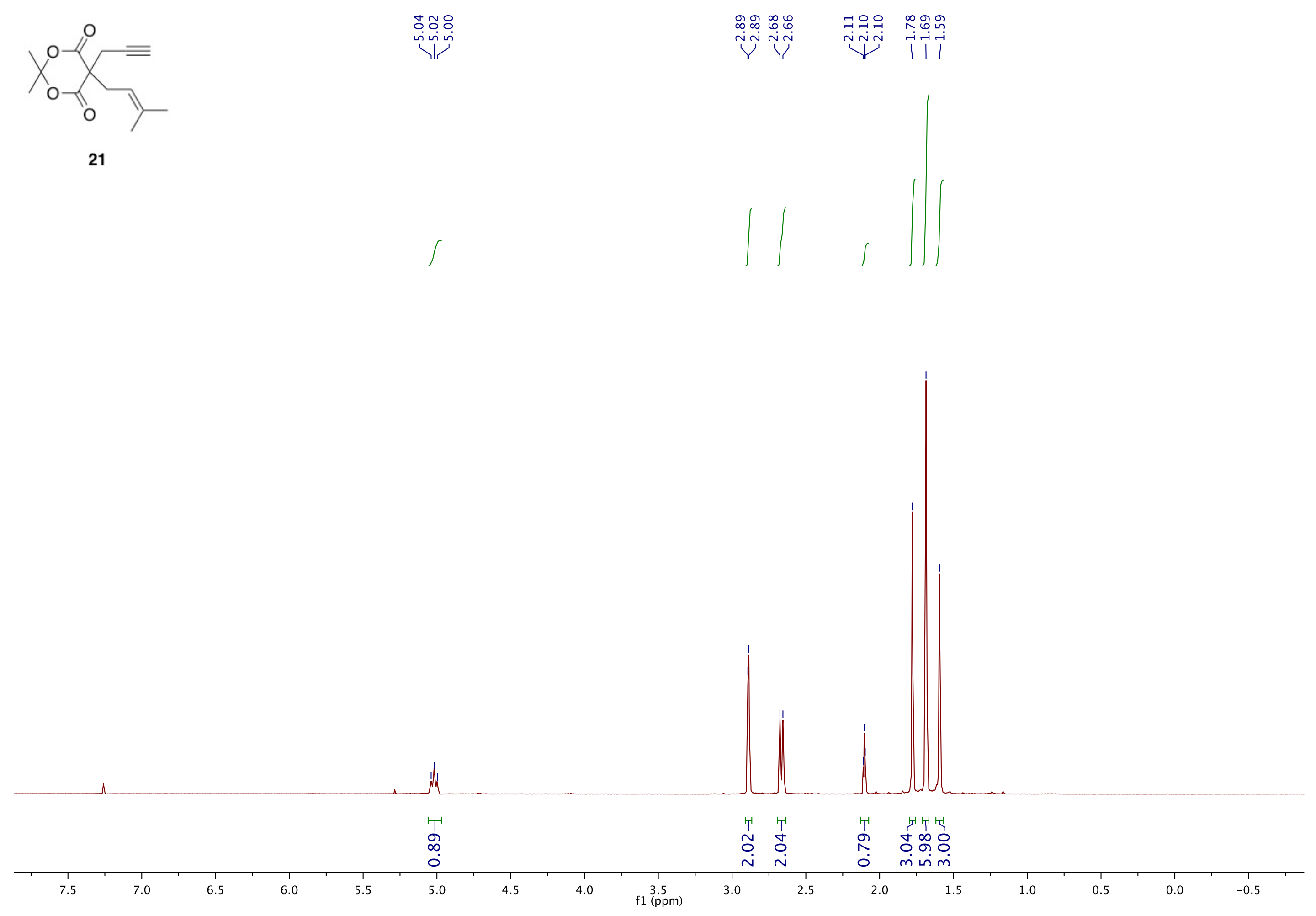


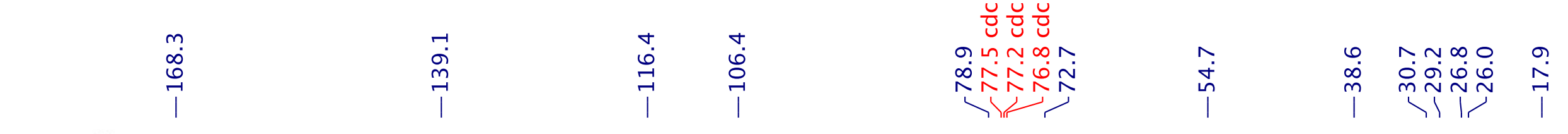

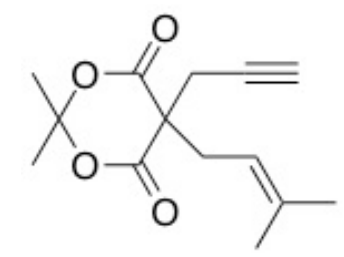

21

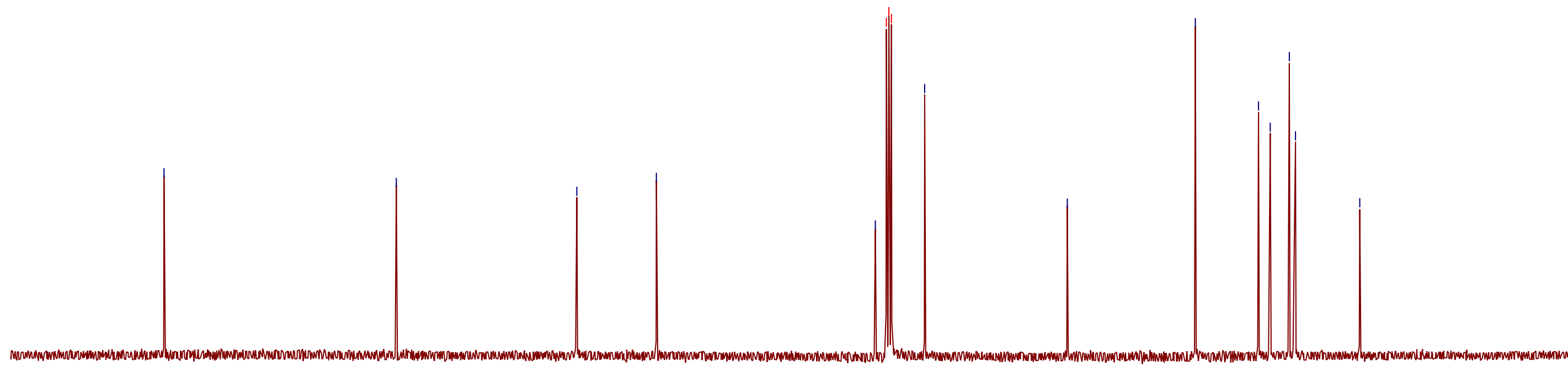

\begin{tabular}{|c|c|c|c|c|c|c|c|c|c|c|c|c|c|c|c|c|c|c|}
\hline 1 & 1 & 1 & 1 & 1 & 1 & 1 & 1 & 1 & 1 & $T$ & $T$ & 1 & $T$ & $T$ & 1 & $T$ & 1 & $T$ \\
\hline 180 & 170 & 160 & 150 & 140 & 130 & 120 & 110 & 100 & f1 $\stackrel{90}{(\mathrm{ppm})}$ & 80 & 70 & 60 & 50 & 40 & 30 & 20 & 10 & 0 \\
\hline
\end{tabular}



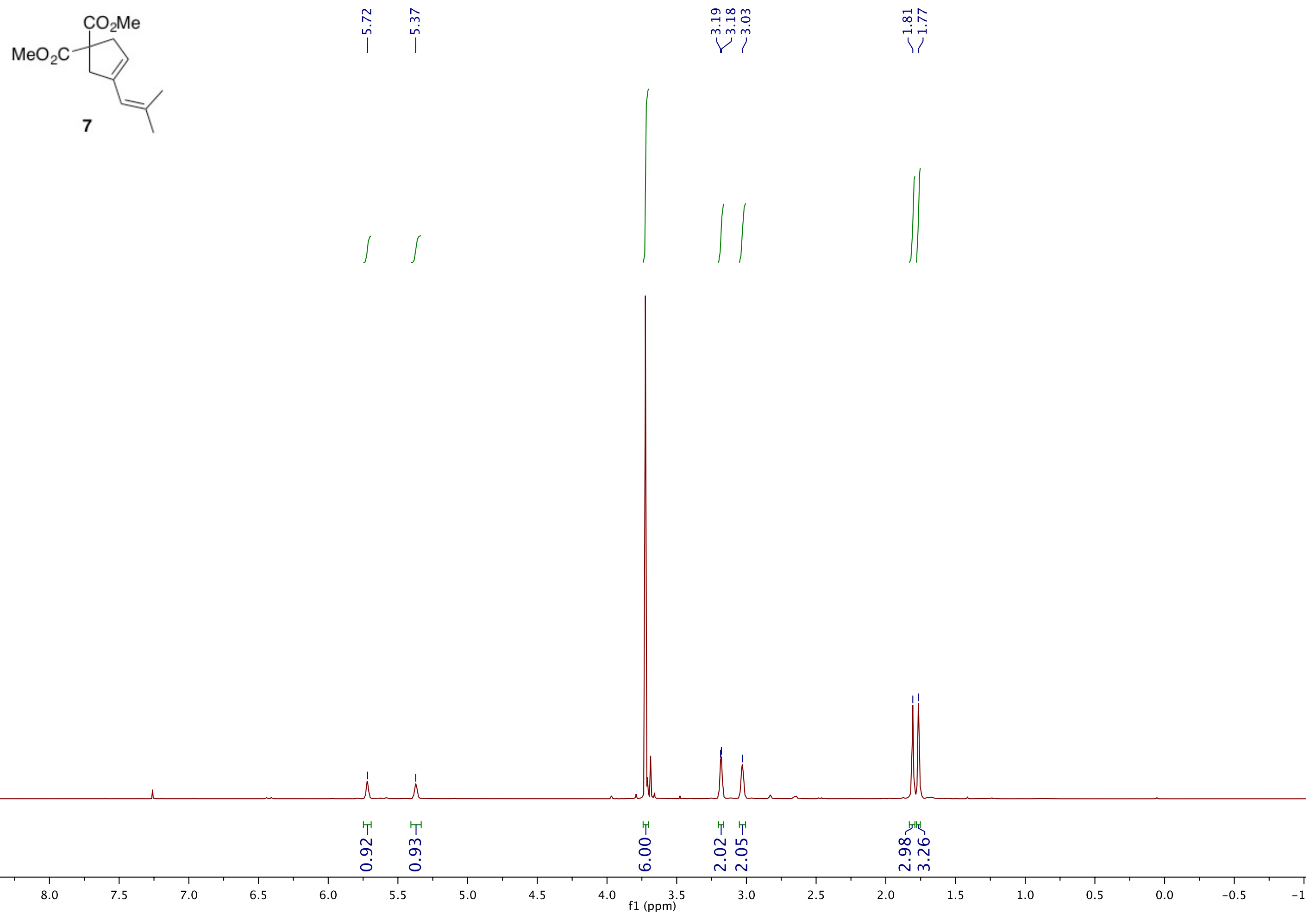


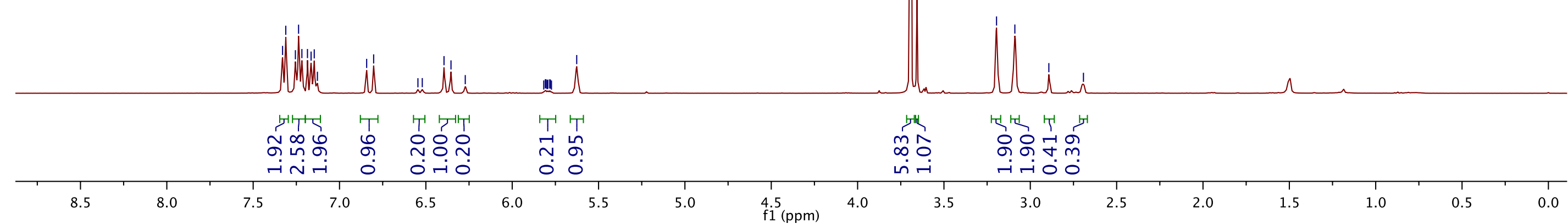




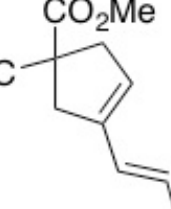

17

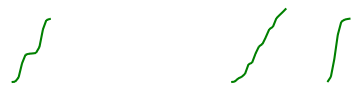

$$
\|
$$
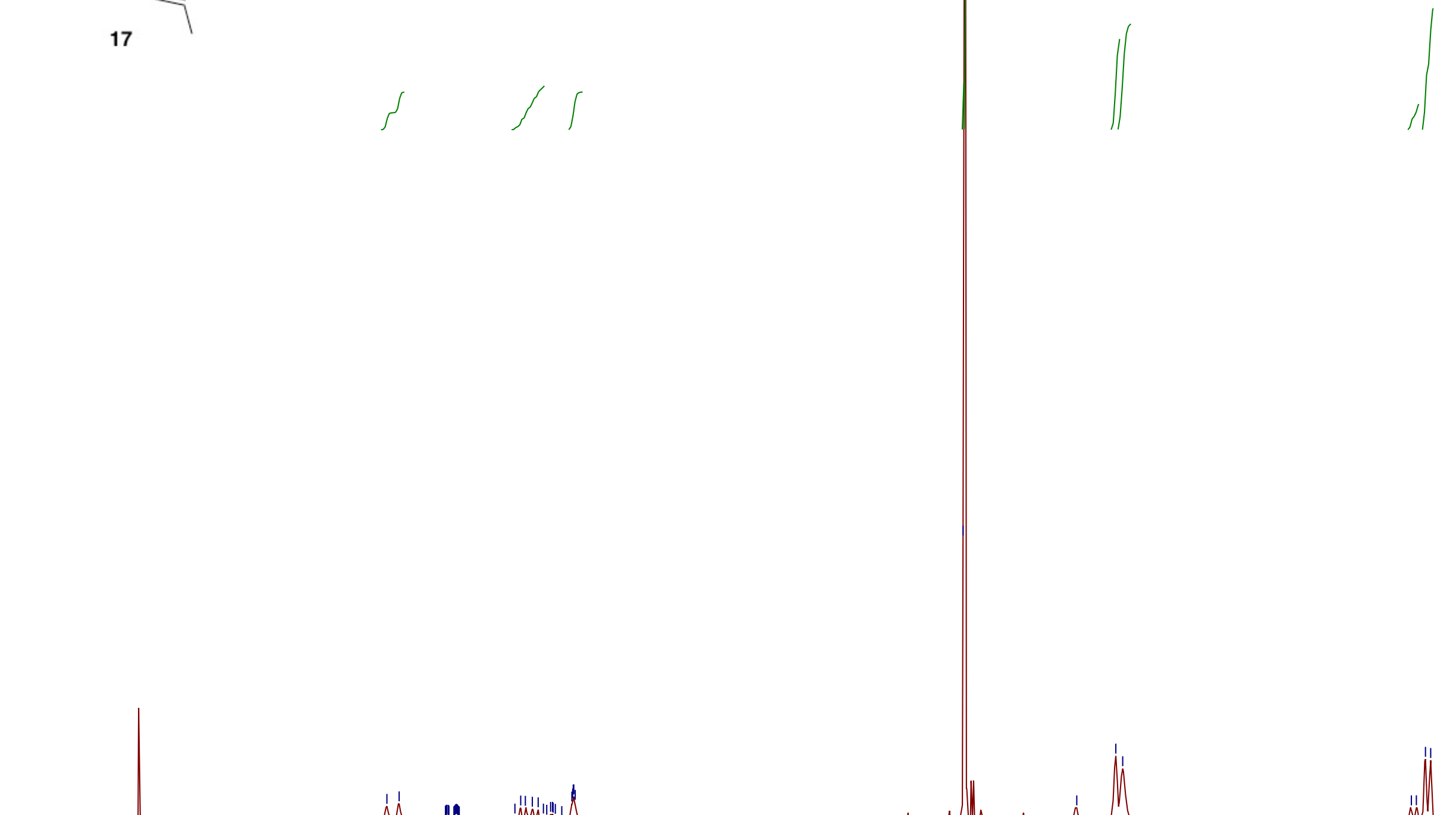

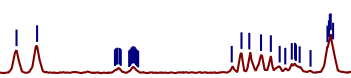
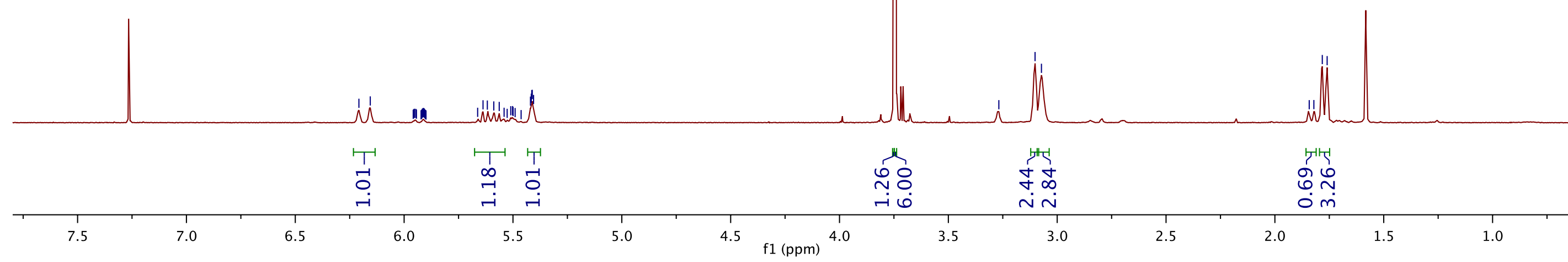


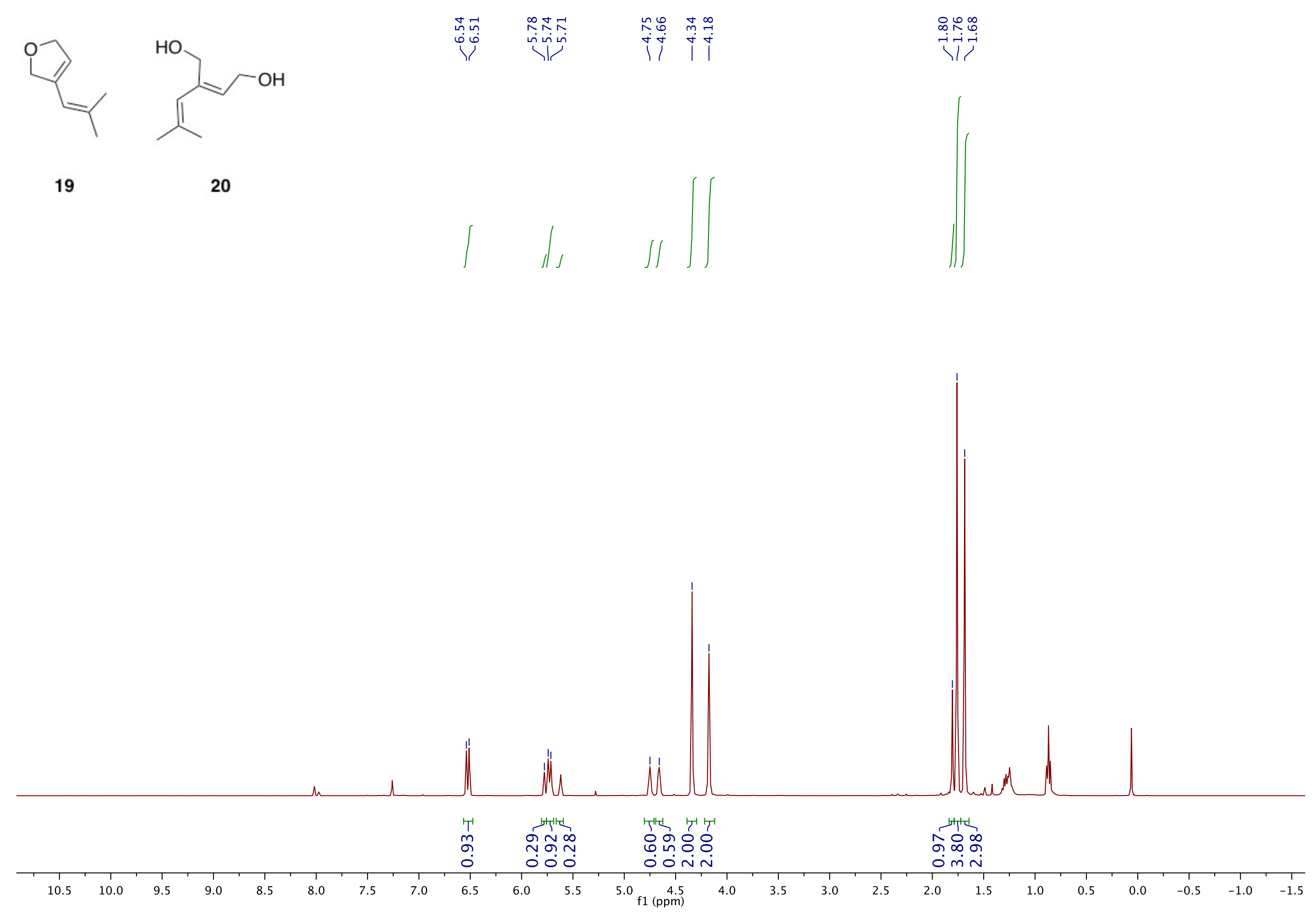



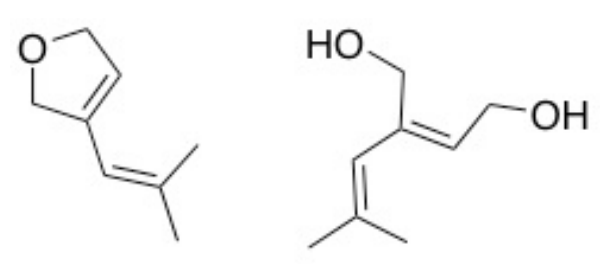

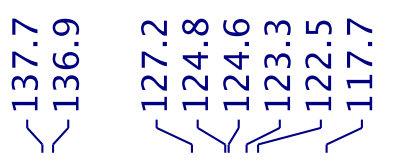

$m \frac{m}{v}$

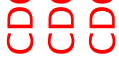

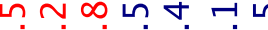

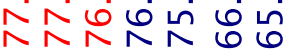

।
N

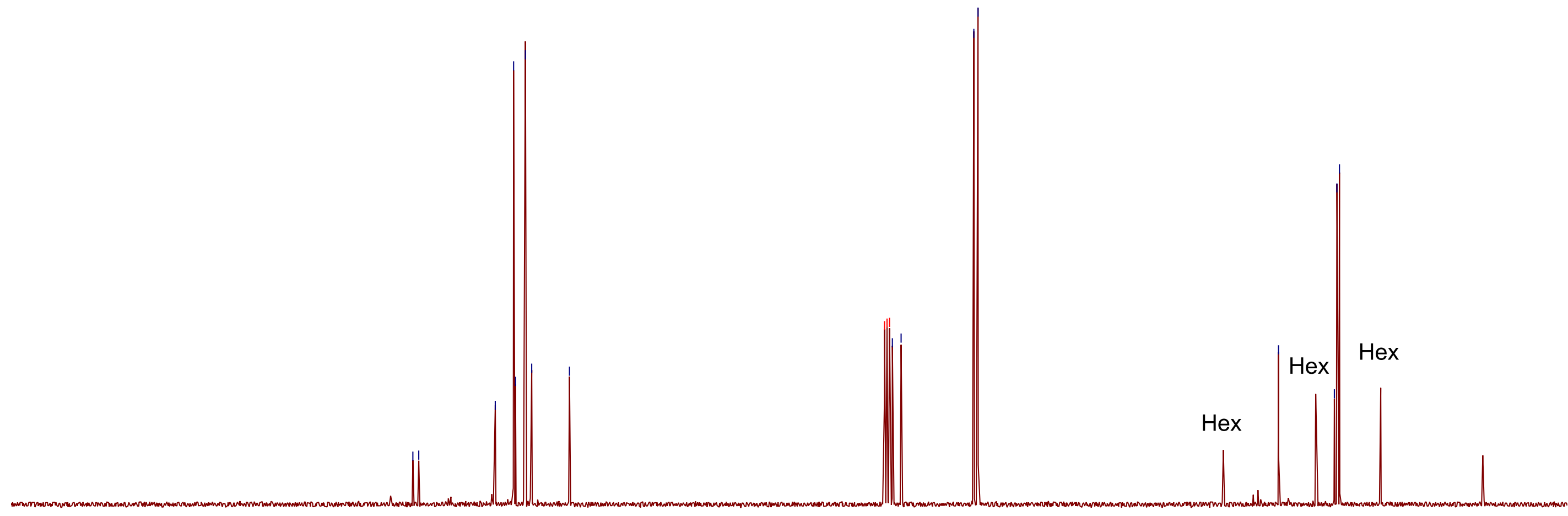



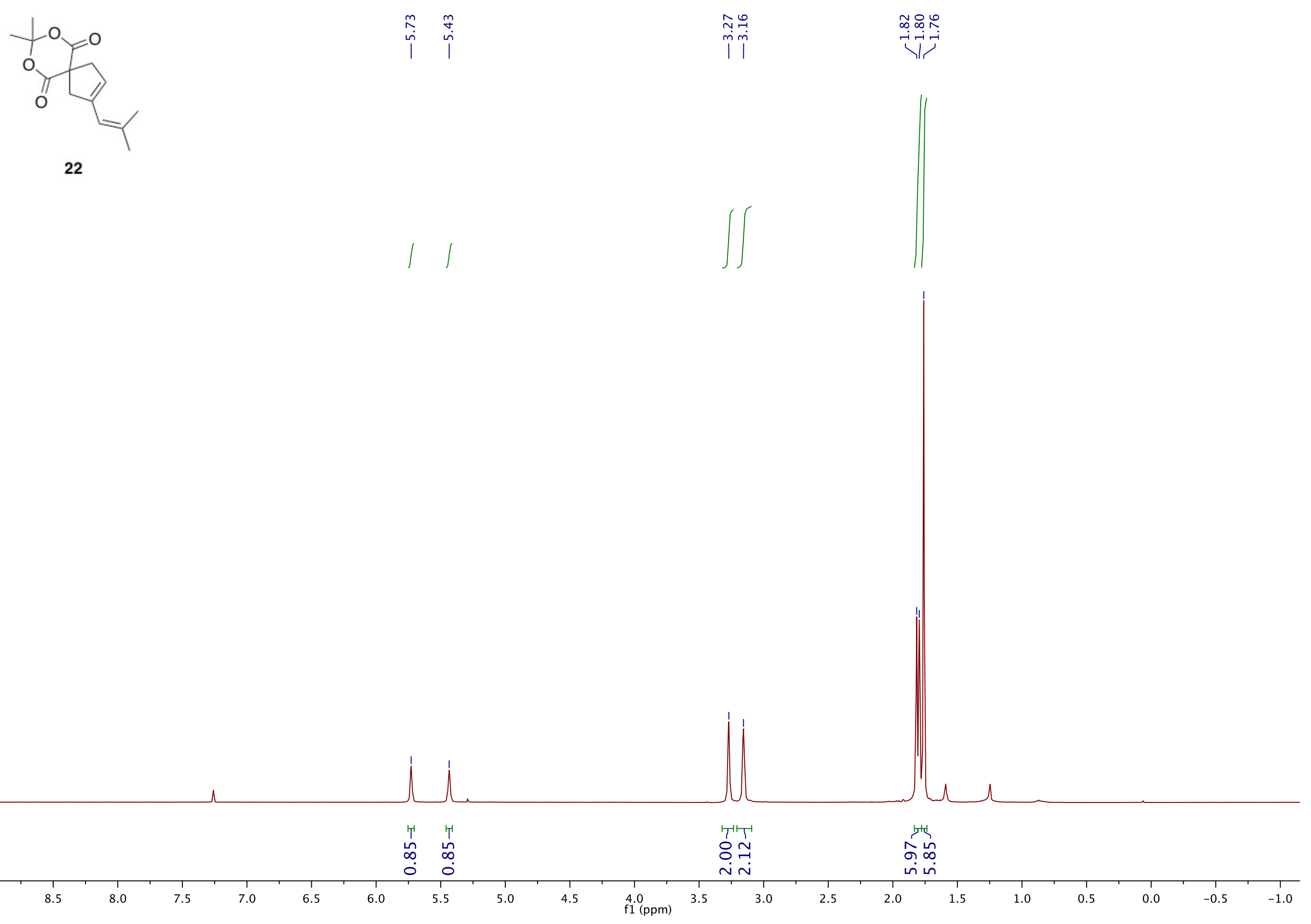


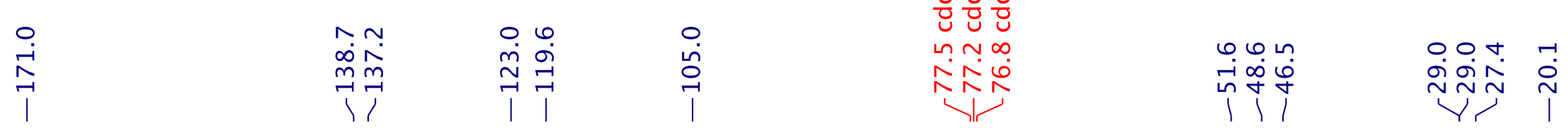

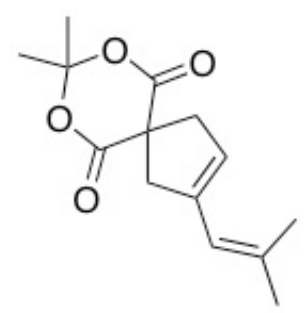

22

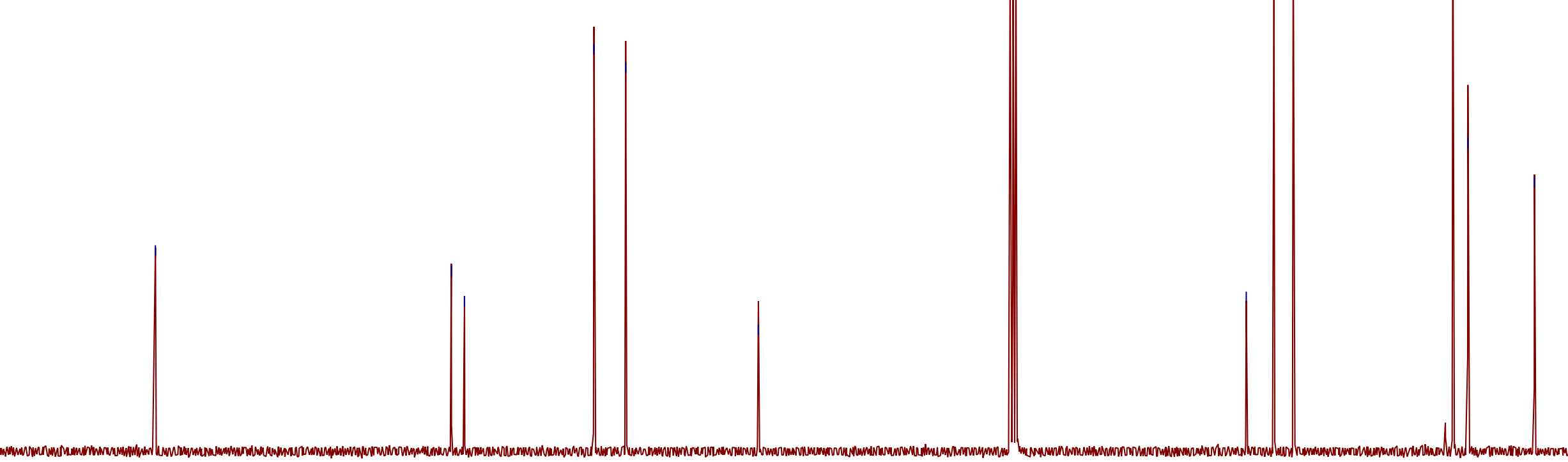

\begin{tabular}{|c|c|c|c|c|c|c|c|c|c|c|c|c|c|c|c|c|c|c|}
\hline 1 & 1 & 1 & 1 & 1 & 1 & 1 & 1 & 1 & 1 & 1 & 1 & 1 & 1 & 1 & 1 & 1 & 1 & \\
\hline 180 & 170 & 160 & 150 & 140 & 130 & 120 & 110 & 100 & f1 $\stackrel{90}{(\mathrm{ppm})}$ & 80 & 70 & 60 & 50 & 40 & 30 & 20 & 10 & 0 \\
\hline
\end{tabular}

